University of Louisville

ThinkIR: The University of Louisville's Institutional Repository

Electronic Theses and Dissertations

$12-2012$

\title{
Gain and loss of functional locomotor recovery following contusive spinal cord injury in the adult rat.
}

Krista Layne Caudle

University of Louisville

Follow this and additional works at: https://ir.library.louisville.edu/etd

\section{Recommended Citation}

Caudle, Krista Layne, "Gain and loss of functional locomotor recovery following contusive spinal cord injury in the adult rat." (2012). Electronic Theses and Dissertations. Paper 225.

https://doi.org/10.18297/etd/225

This Doctoral Dissertation is brought to you for free and open access by ThinkIR: The University of Louisville's Institutional Repository. It has been accepted for inclusion in Electronic Theses and Dissertations by an authorized administrator of ThinkIR: The University of Louisville's Institutional Repository. This title appears here courtesy of the author, who has retained all other copyrights. For more information, please contact thinkir@louisville.edu. 


\title{
GAIN AND LOSS OF FUNCTIONAL LOCOMOTOR RECOVERY FOLLOWING CONTUSIVE SPINAL CORD INJURY IN THE ADULT RAT
}

\section{By}

Krista Layne Caudle

B.S. University of North Carolina at Charlotte, 2004/2006

M.S. University of Louisville, 2008

\author{
A Dissertation \\ Submitted to the Faculty of the \\ School of Medicine of the University of Louisville \\ In Partial Fulfillment of the Requirements \\ For the Degree of
}

Doctor of Philosophy

Department of Anatomical Sciences and Neurobiology

University of Louisville

Louisville, Kentucky

December 2012 
Copyright 2012 by Krista Layne Caudle

All rights reserved. 
GAIN AND LOSS OF FUNCTIONAL LOCOMOTOR RECOVERY FOLLOWING CONTUSIVE SPINAL CORD INJURY IN THE ADULT RAT

\author{
By
}

Krista Layne Caudle

B.S. University of North Carolina at Charlotte, 2004/2006

M.S. University of Louisville, 2008

A Dissertation Approved on

November 14, 2012

by the following Dissertation Committee

David S.K. Magnuson, Ph.D.

Martha Bickford, Ph.D.

Susan Harkema, Ph.D.

Michael Voor, Ph.D.

Scott Whittemore, Ph.D. 


\section{ACKNOWLEDGMENTS}

I would like to thank Dr. David S.K. Magnuson for mentorship in science and personal development, members of the Magnuson Laboratory, and KSCIRC Core Facilities for their assistance and expertise. I especially want to thank Alice Shum-Siu, Darlene Burke, Edward Brown, Johnny Morehouse, and Darryn Atkinson for helping with experiments and data analysis, day in and day out. I would also like to thank my committee members for their time and valuable input during the dissertation project. Dr. Scott Whittemore and Dr. Susan Harkema have been invaluable resources for showing me the spectrum of issues involving spinal cord injury. Finally, I would like to thank Dr. David P. Bashor for teaching me the foundations of neuroscience and helping me keep the privilege of science top of mind. 


\title{
ABSTRACT \\ GAIN AND LOSS OF FUNCTIONAL LOCOMOTOR RECOVERY FOLLOWING CONTUSIVE SPINAL CORD INJURY IN THE ADULT RAT
}

\author{
Krista Layne Caudle
}

November 14, 2012

Activity-based rehabilitation in the form of overground or body weightsupported treadmill (BWST) locomotor step training has become the most widely accepted therapy translated from preclinical animal research to spinal cord injury (SCI) patients. However, locomotor training does not provide the level of functional locomotor recovery that animal models are interpreted to promise because preclinical studies have used complete spinal cord transections that do not sufficiently mimic the clinical presentation. Furthermore, animal models do not include the same standard of care, immobilization with stretch/range-ofmotion manual therapies, $\mathrm{SCl}$ patients receive. Therefore, we have developed an experimental animal model that includes aspects of acute patient care, immobilization and manual therapy interventions, applied daily throughout the 8 weeks following incomplete low thoracic contusion $\mathrm{SCl}$ in adult rats. We hypothesize that laboratory animals with clinically relevant incomplete contusion $\mathrm{SCl}$ achieve maximal locomotor recovery while moving about in their cages, "auto-training," within the first few weeks post-injury. Our results show that when immobilization and/or manual therapy interventions are applied the animals suffer 
severe short-term loss of locomotor function that significantly limits potential for long-term recovery even weeks after the interventions end. Our studies suggest that immobilization and widely practiced manual therapies may be maladaptive for functional locomotor recovery after clinically relevant incomplete $\mathrm{SCl}$. 


\section{TABLE OF CONTENTS}

PAGE

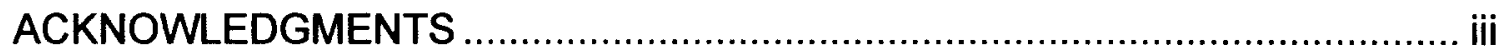

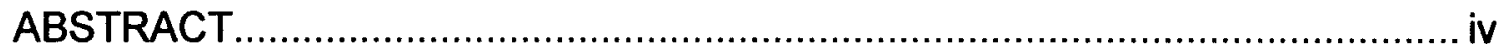

LIST OF FIGURES

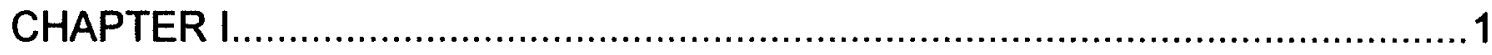

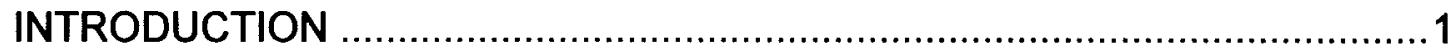

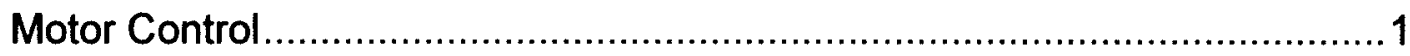

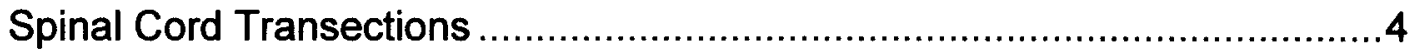

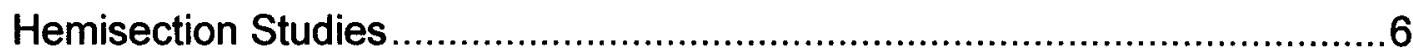

Clinically Relevant Spinal Cord Injury .................................................10

Activity-Based Training after Incomplete $\mathrm{SCl}$.....................................12

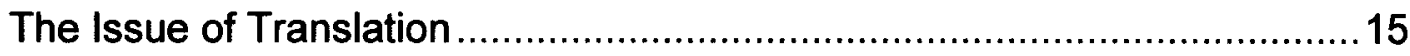

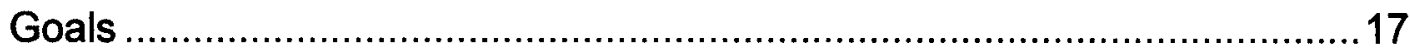

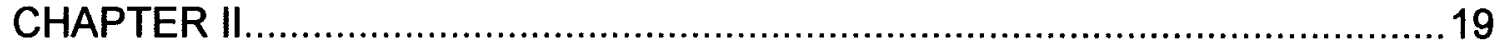

HINDLIMB IMMOBILIZATION IN A WHEELCHAIR ALTERS FUNCTIONAL RECOVERY FOLLOWING CONTUSIVE SPINAL CORD INJURY IN THE

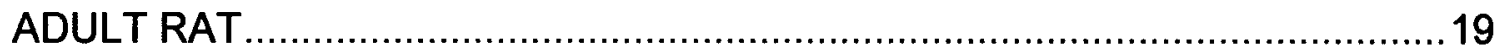

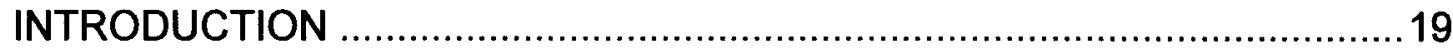

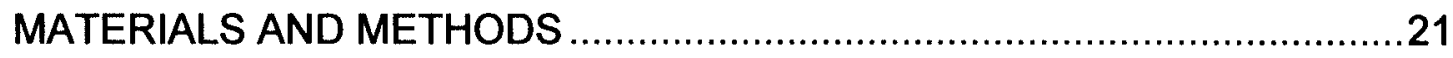

Spinal Cord Injury and Experimental Design .........................................21

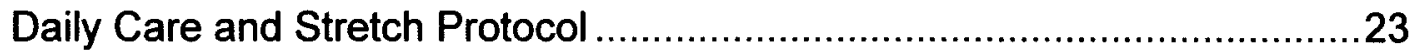

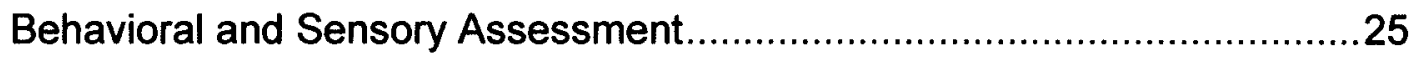


Kinematic Assessment of Stepping and Swimming ................................27

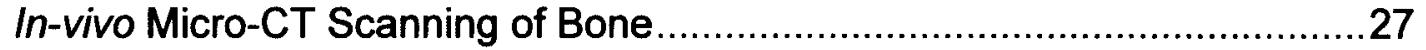

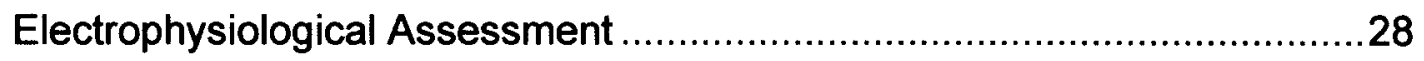

Histology of Sciatic Nerve and Spinal Cord Injury Epicenter......................30

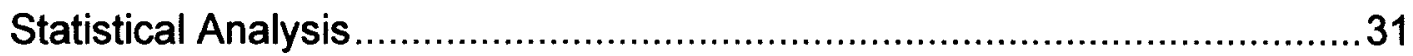

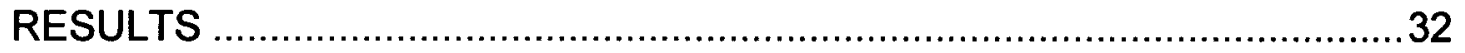

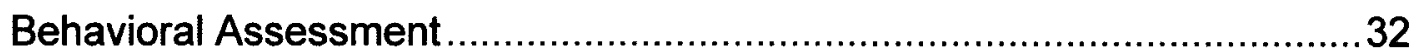

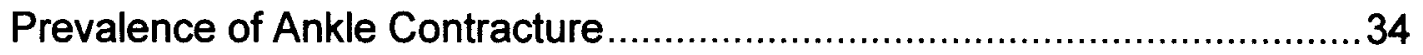

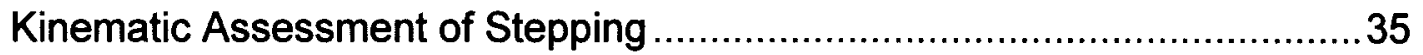

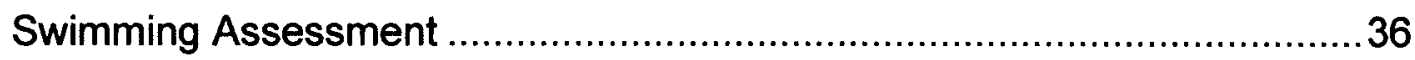

In-vivo Micro-CT Scanning of Hindlimb Bone...............................................38

Body, Muscle Weight and Sciatic Assessment .........................................39

EMG Recordings of Gastrocnemius Muscle ............................................40

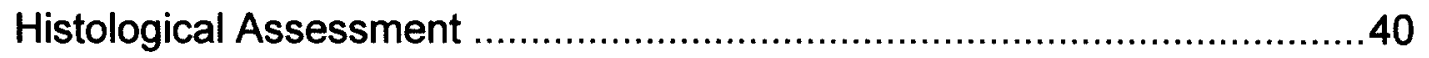

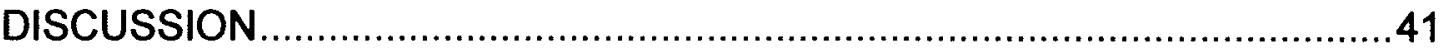

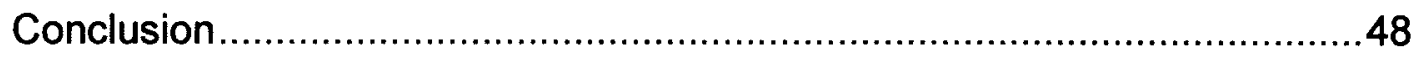

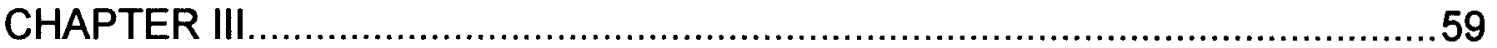

FUNCTIONAL LOCOMOTOR RECOVERY IS NEGATIVELY ALTERED IN A TASK-SPECIFIC MANNER FOLLOWING CONTUSIVE SPINAL CORD INJURY

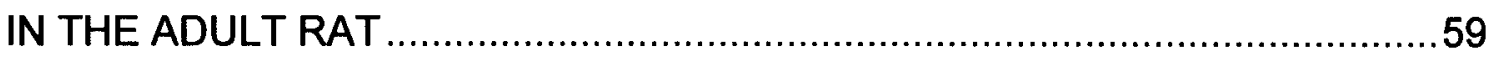

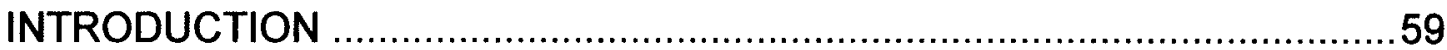

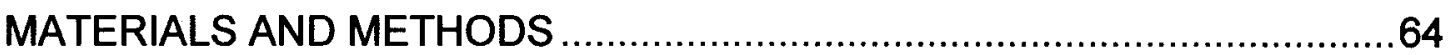

Spinal Cord Injury and Experimental Design ...........................................64

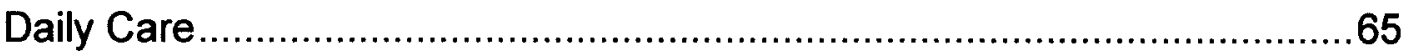

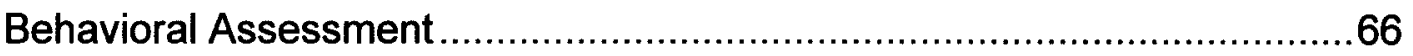


ROM of the Ankle

Kinematic Assessment of Stepping, Swimming and Shallow Water

Walking. 68

Ex-vivo Micro-CT Scanning of Hindlimb Bone ............................................

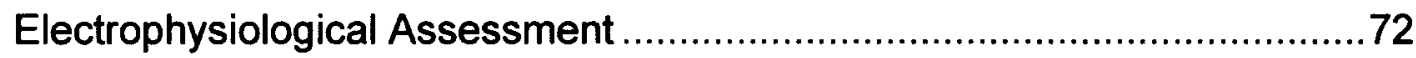

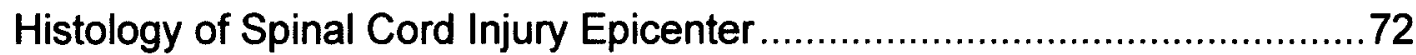

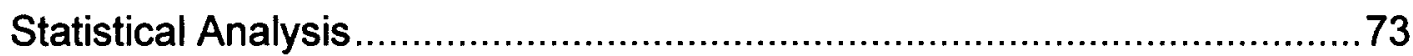

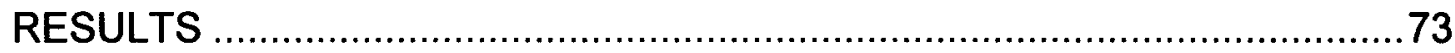

Behavioral Assessment Recovery of Hindlimb Locomotion .........................73

Kinematic Assessment of Intralimb Coordination During Overground

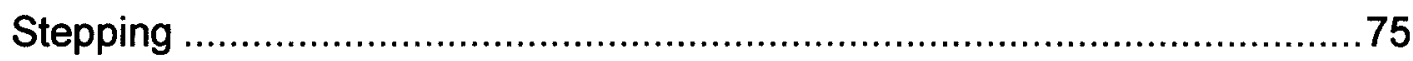

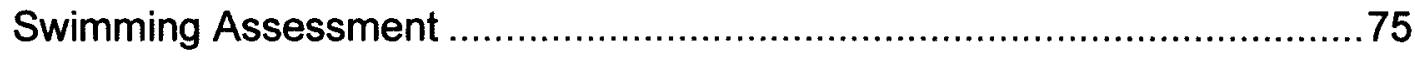

Hindlimb Movements While in Wheelchairs ...........................................76

Kinematic Assessment During Shallow Water Walking ...........................77

Interlimb Coordination and Plantar Stepping During Shallow Water

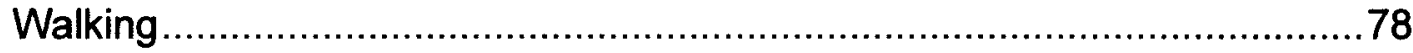

Interlimb Coordination Coupling During Shallow Water Walking ................78

EMG Recordings of Muscles Controlling the Ankle.................................79

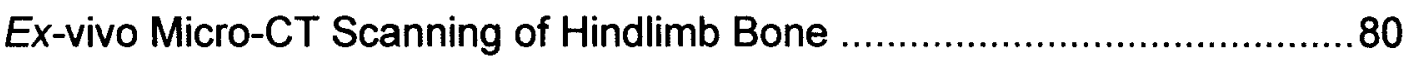

Body, Muscle Weight and Histological Assessment..................................8

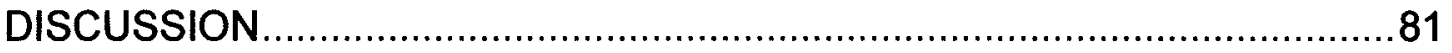

Task-specificity and Locomotor Recovery ........................................... 81

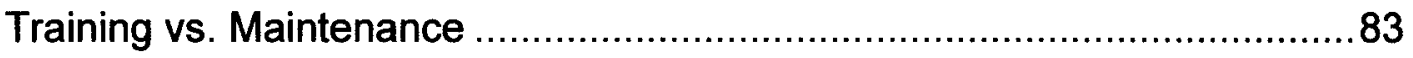

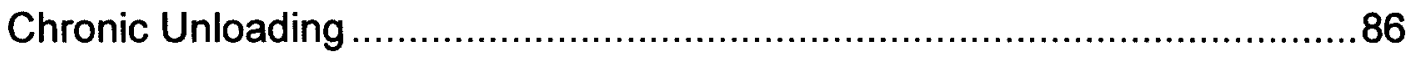


Conclusion

THERE IS A NEGATIVE EFFECT OF HINDLIMB STRETCH ON LOCOMOTOR RECOVERY FOLLOWING CONTUSIVE SPINAL CORD INJURY IN THE ADULT RAT.

INTRODUCTION 103

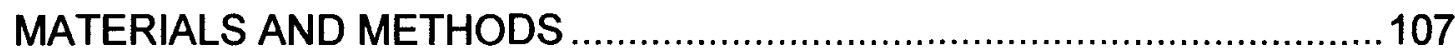

Spinal Cord Injury and Experimental Design .......................................107

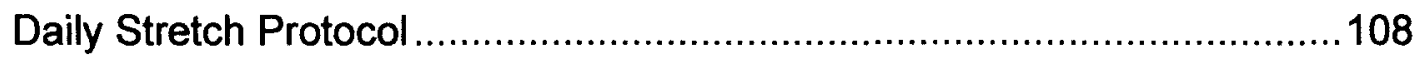

Immediate Stretch Response (ISR) Scores ......................................109

Goniometer Measurements of Passive ROM .....................................110

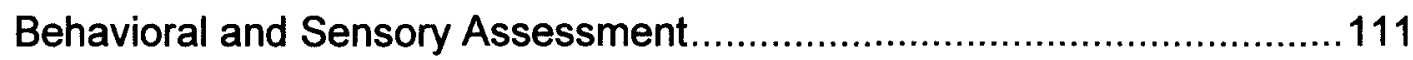

Kinematic Assessment of Stepping and Shallow Water Walking ...............112

Magnetic Evoked Potentials from Tail Stimulation .................................113

Histology of Spinal Cord Injury Epicenter ............................................114

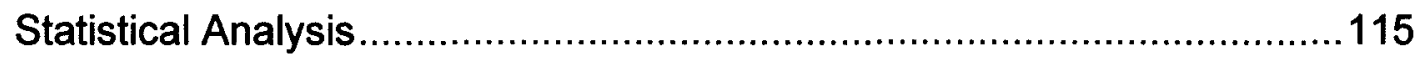

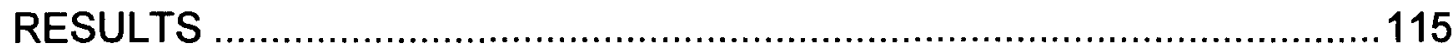

Behavioral Assessment Recovery of Hindlimb Locomotion ......................115

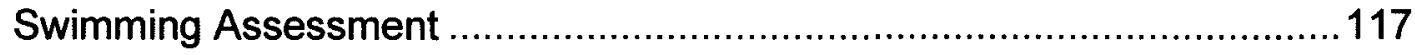

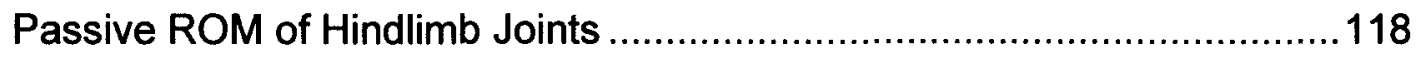

Kinematic Assessment of Intralimb Coordination During Overground Stepping

Interlimb Coordination and Plantar Stepping During Shallow Water Walking.

Immediate Stretch Responses (ISR) and BBB.....................................121

Magnetically Evoked Hindlimb Muscle Responses ................................124 
Body, Muscle Weight and Histological Assessment...............................124

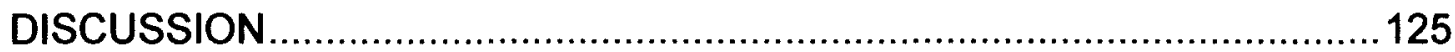

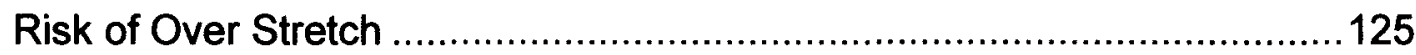

Relationship of pROM and Overground Joint Excursion Kinematics .........127

Gain and Loss of Locomotor Function ...........................................128

Stretching and Locomotor Function ................................................132

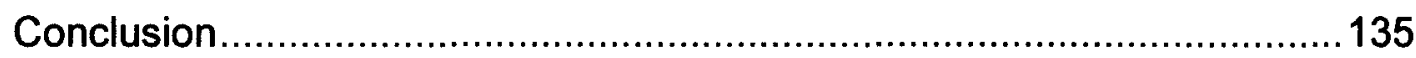

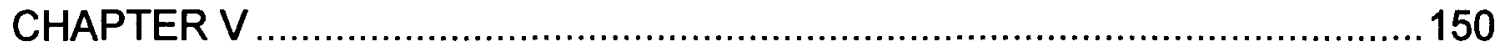

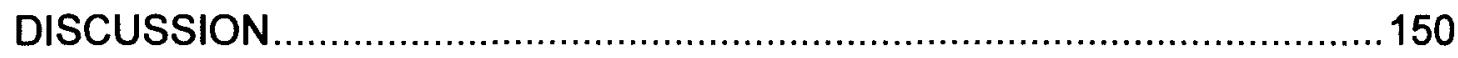

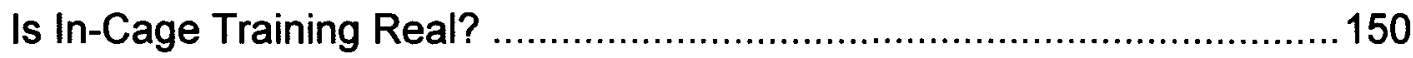

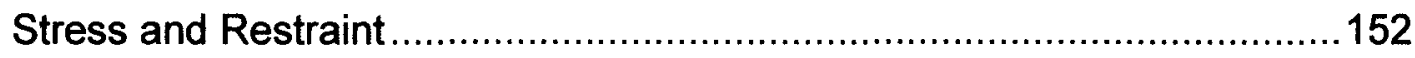

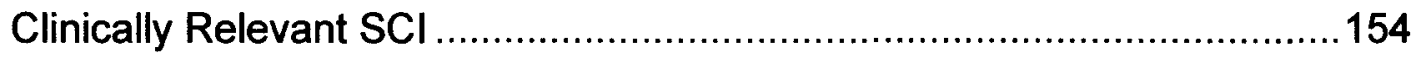

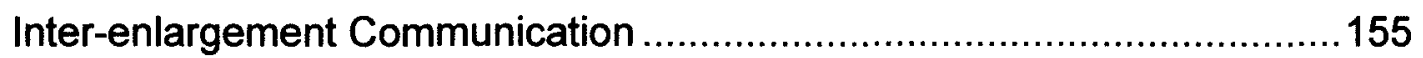

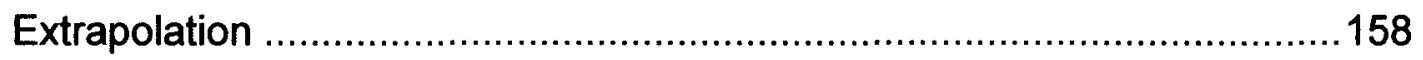

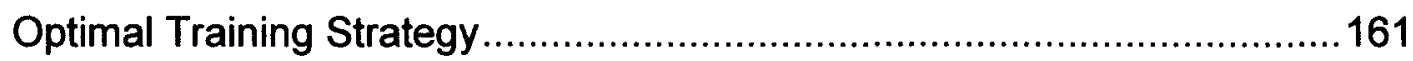

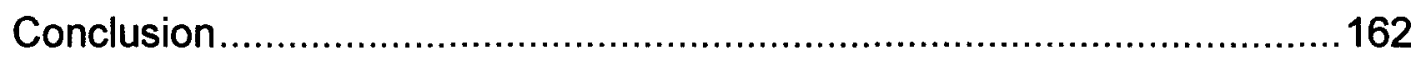

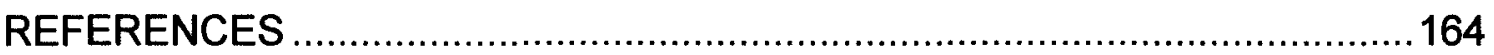

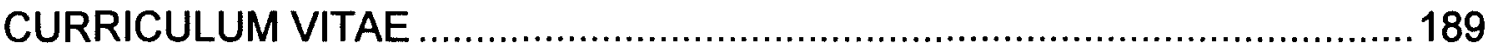




\section{LIST OF FIGURES}

Figure 1. Timeline WC Hindlimb Immobilization...........................................49

Figure 2. BBB Scores WC Hindlimb Immobilization. ....................................50

Figure 3. Intralimb Coordination Overground Stepping WC Hindlimb

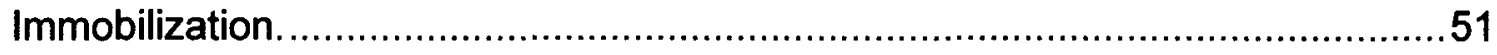

Figure 4. Swimming Assessments WC Hindlimb Immobilization. .....................53

Figure 5. In-vivo Micro-CT Bone Architecture WC Hindlimb Immobilization. ......55

Figure 6. Muscle Weight WC Hindlimb Immobilization...................................56

Figure 7. Muscle Co-contraction WC Hindlimb Immobilization.........................57

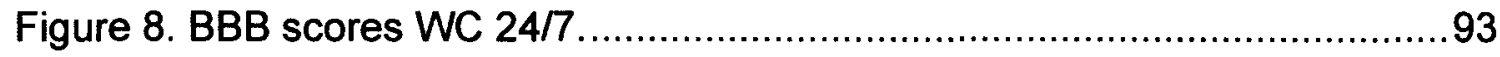

Figure 9. Intralimb Coordination Overground Stepping WC 24/7 ....................94

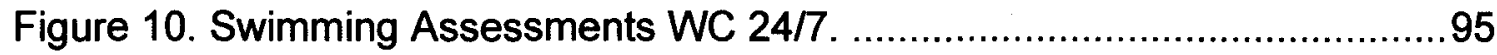

Figure 11. Hindlimb Movements While in WC 24/7 ........................................96

Figure 12. Intralimb Coordination Shallow Water Walking WC 24/7 ................97

Figure 13. Pattern Formation and Plantar Stepping During Shallow Water

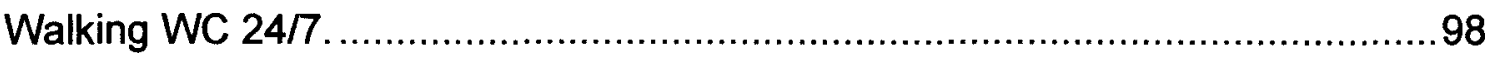

Figure 14. Interlimb Coupling Shallow Water Walking WC 24/7. .....................99

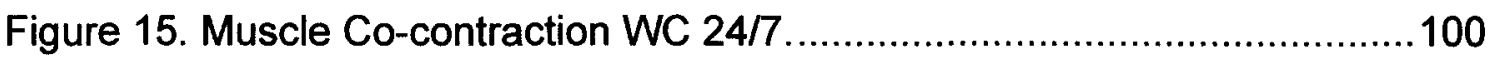

Figure 16.. Ex-Vivo Micro-CT Hindlimb Bone Architecture WC 24/7...............101

Figure 17. Timeline 8 Weeks Daily Stretch. …….........................................136

Figure 18. 3D Kinematic Representation of Hindlimb Stretch Positions...........137 
Figure 19. BBB Scores Daily Stretch........................................................

Figure 20. Swimming Assessments Daily Stretch......................................142

Figure 21. Passive range-of-motion (pROM) for Hindlimb Joints Daily Stretch. 143

Figure 22. Intralimb Coordination Overground Stepping Daily Stretch............. 144

Figure 23. Pattern Formation and Plantar Stepping Shallow Water Walking Daily

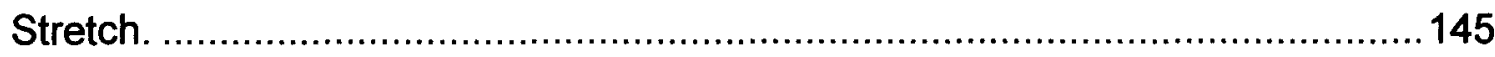

Figure 24. Immediate Stretch Responses (ISRs) Daily Stretch......................146

Figure 25. Magnetically Evoked Muscle Responses Daily Stretch...................148 


\section{CHAPTER I}

\section{INTRODUCTION}

\section{Motor Control}

Early discoveries about motor control were conducted in isolated nervous systems of crayfish, locust, and lamprey, but studies in mammals began with the traditional decerebrate cat preparations using a pre-collicular transection (Mulloney and Smarandache, 2010). In isolated spinal cord "fictive" motor preparations electrical or pharmacological stimulation evokes the rhythmic pattern of locomotion, but motor neurons and primary afferent dorsal root ganglia neurons are no longer innervating the hindlimbs; root recordings are representative of the actual motor output of the isolated spinal cord circuitry. Both the speed of locomotion and the gait pattern are sensitive to the intensity, but not the frequency, of stimulation in the mesencephalic locomotor region (MLR) (Rossignol, 1996; Rossignol et al., 1996). The subthalamic locomotor region (SLR) and MLR are responsible for activation of the pontomedullary medial reticular formation (RF) that projects, via reticulospinal tracts, caudally to the lumbar enlargement to "drive" locomotion and postural support (Jordan and Schmidt, 2002). When the intensity of brainstem stimulation is increased, the decerebrate animal goes from walking to trotting, and then to galloping. Pontomedullary induced locomotion is dependent on intact dorsal columns (DC) and dorsolateral funiculi (DLF); lesions of these pathways may not totally block 
locomotion, but may result in toe drag or difficulty maneuvering over obstacles during treadmill stepping (Rossignol, 1996). These studies indicate that . supraspinal centers control the initiation of locomotion and are powerful modulators of both speed and gait.

Decerebrate animals can also be induced to step by afferent stimulation alone. Tonic stimulation of dorsal roots can evoke fictive locomotion in decerebrate preparations, and just treadmill movement or perineal stimulation can initiate locomotion in animals that are spinalized at lower levels of the spinal cord. However, using an injection of dihydroxyphenylalanine to drive activity, Grillner showed that the basic locomotor pattern is still expressed even after dorsal rhizotomy (removal of all phasic sensory afferent input) (Grillner and Zangger, 1979). The fact that a basic locomotor pattern can be generated in the absence of cortical input and movement related sensory feedback provides direct evidence of the independent capacity for locomotion in spinal structures. A central pattern generator (CPG) is a relatively small and autonomous network of neuron types that has the endogenous ability to produce stereotypic yet complex rhythmic patterned motor output in the absence of afferent sensory feedback (Rossignol, 1996). Central pattern generators have been described for respiration, swallowing, pyloric contractions, heartbeat, vibrissa whisking, scratching, swimming, flying and stepping (Rossignol et al., 1996; Hooper, 2000; Mulloney and Smarandache, 2010). Rhythm generation is the timing and duration of cycles of movement, and is expressed behaviorally as the speed of locomotion. Pattern formation is expressed behaviorally as the footfall sequence 
of the gait/interlimb coordination, the relationship of the joint angles, and the resulting trajectory of a single limb/intralimb coordination.

Traditional studies established that the isolated CPG for locomotion, by definition, does not require afferent input to produce rhythmic locomotor activity of the hindlimbs; however, in an intact central nervous system (CNS) the CPG's role is to integrate afferent information with goal directed commands from the cortex and brain stem structures. The main function of afferent input on the hindlimb locomotor CPG is to ensure proper excitation of extensors during weight bearing activities, proper timing of muscle drive in relation to the direction of the moving body, and control of the phase transitions and smoothness of gait in order to maintain balance (Majczynski and Slawinska, 2007). Spinal reflexes are the mechanisms by which sensory information can modify motor activity both by direct action on motor neurons or indirectly through interneurons of the CPG (Rossignol et al., 1996; Barbeau et al., 1999b). Homonymous and closely synergistic muscle groups are wired together via monosynaptic excitation with disynaptic inhibition of antagonist muscle groups, and are evoked by 1a primary muscle spindle afferents that synapse directly onto alpha motor neurons (Barbeau et al., 1999b). Group 1a primary afferents are activated by increases in muscle length/stretch or by the gamma motor neuron system that regulates muscle spindle sensitivity; both encode information about proprioception. Group II secondary muscle spindle afferents evoke flexion reflexes directly and through interneurons, but also interact with descending motor commands and are considered an essential part of the moment-by-moment regulation of movement. 
Golgi tendon organs within muscles, known as non-reciprocal $1 \mathrm{~b}$ afferents, are highly sensitive to muscle stretch and tendon tension and encode information about load (Barbeau et al., 1999b). Cutaneous reflexes are generally weak and can be local, affecting only the immediately surrounding innervated muscles; or exerted extensively affecting the entire limb. Reflex actions are state-dependent in that the response to afferent input can be opposite based on the current motor task. For example, if Group 1 afferents are stimulated during the flexion swing phase of stepping, flexor activity is terminated while extensor activity is initiated, and the step cycle is reset. However, if the stimulation occurs during extension or stance phase then extensor activation is prolonged (Barbeau et al., 1999b). The complex integration involved with production and control of locomotion can be appreciated with spinal lesion studies in animal models in which the fundamental components of the CPG, rhythm and pattern, can be augmented by afferent input applied with the goal of training stepping (Rossignol et al., 1996; Cai et al., 2006a; Majczynski and Slawinska, 2007).

\section{Spinal Cord Transections}

Studies of body weight-supported treadmill (BWST) step training after complete thoracic transection in adult cats (Rossignol et al., 2004), rats (de Leon and Acosta, 2006; Zhang et al., 2007; Ichiyama et al., 2008), and mice (Leblond et al., 2003; Cai et al., 2006b), and after severe incomplete lesions in non-human primates (Eidelberg et al., 1981; Vilensky et al., 1992) have revealed a robust capacity for locomotor recovery, presumably via accessing or training the CPG. Step training attempts to supply the proper afferent cues to the nervous system 
to induce the expression of a basic locomotor pattern that can be fine-tuned with practice to result in functional locomotor recovery. Studies demonstrate that each type of afferent information, cutaneous afferents, joint capsules, and muscle spindles encoding information for proprioception and muscle load, elegantly contributes to motor control and can be tested in isolation (Edgerton et al., 2004).

Cats spinalized at thoracic spinal level therteen (T13), standing with the forelimbs on a stationary platform with lateral balance support provided manually, can maintain plantar paw contact and support body weight with extensor activity, and can be stand or step trained (Barbeau et al., 1999b; Rossignol et al., 2002; Windhorst, 2007). With handler support of the tail at least initially, perineal stimulation, and daily step training on the treadmill, after a few weeks these cats can perform full weight-supported stepping with only minor deviations from normal in pattern or toe clearance (Barbeau and Rossignol, 1987). Step trained spinalized animals can learn to generate symmetrical consecutive steps without stumbling, achieve overall angular excursions of hip, knee, and ankle that are nearly normal (Lovely et al., 1986; Belanger et al., 1996), and have considerably faster speed of stepping with enhanced flexor and extensor activation as compared to untrained spinalized animals (de Leon et al., 1998b). The stepping performance is of higher quality with greater amounts of practice and repetition (Lovely et al., 1986; Edgerton et al., 1991; Cha et al., 2007; Dobkin et al., 2007). The trajectory and timing of the entrained step can be modulated by perturbations of proprioceptive, cutaneous and load afferents during stepping. A coordinated hyperflexion can bring the hindpaw up and over an obstacle that 
catches the dorsum of the paw during the swing phase indicating that cutaneous input plays an important role in reflex responses during swing (Forssberg, 1979; Quevedo et al., 2005). Elimination of cutaneous input from both sides of the hindpaw produces only minor changes in stepping pattern that are resolved quickly with step training. However, if all cutaneous input is systematically eliminated after progressive denervation, even a highly extended regimen of several months of step training is unsuccessful for recovery of proper weightsupported plantar stepping (Bouyer and Rossignol, 2003a, b). Therefore, even very little sparing of hindpaw plantar cutaneous afferents is both necessary and sufficient for correct foot placement and weight support when supraspinal control is eliminated. Phasic cutaneous input does not have to be coupled to weight support or plantar placement in order to bring about a change in the trajectory of the hindlimb. In a chick hemisection model, cutaneous input supplied during swim training increased the extensor activity of the retraction phase of the swim cycle indicating that a repeated pattern of cutaneous input altered the trajectory of the limb in a task-specific manner that did not also require extensor load weight support (Muir and Steeves, 1995). Low thoracic transection studies in cats (Edgerton et al., 1992) and rats (Timoszyk et al., 2005) have also underscored phasic loading of weight bearing muscles during stance as a critical component of step training. Handlers can increase extensor loading by pulling down on the tail of the animal during step training; the effect is increased amplitude and duration of EMG bursts in extensor muscle groups. Hemisection Studies 
There are anatomical differences in the spinal cord pathways controlling locomotion among rats, cats and humans. The corticospinal tract (CST) is necessary for fine motor tasks in rats and mice, but is of little importance for stereotypic overground stepping. The majority of reticulospinal projections travel in the ventrolateral funiculus (VLF); even very little sparing of VLF preserves the ability to voluntarily initiate coordinated, weight-supported overground stepping in cats and rats (Basso et al., 2002; Schucht et al., 2002; Loy et al., 2002a). However in humans, the CST is of paramount importance for overall locomotor capabilities. Localized distribution of CPG neurons differs from species to species. The CPG for locomotion in rats is located primarily in the rostral segments of the lumbar enlargement, T13-L2 (Cazalets et al., 1995; Cowley and Schmidt, 1997; Magnuson and Trinder, 1997), L2-L4 in the cat (Rossignol et al., 2002; Langlet et al., 2005) and in primates/humans the CPG for locomotion is thought to extend over a larger portion of the lumbar segments, L1-L5 (Gerasimenko et al., 2007; Gerasimenko et al., 2008). An injury that disrupts all or part of the CPG itself may not be comparable across species, and investigators must be careful not to over interpret results without careful consideration of the precise location of the lesion.

The spinal enlargements are interconnected by highly commissural long propriospinal neurons (Reed et al., 2008; Reed et al., 2009) responsible for interlimb coordination. As reviewed by Jordan and Schmidt, the propriospinal interneuron system is situated throughout the rostrocaudal extent of the spinal cord, and receives descending locomotor command signals from collaterally 
sprouting reticulospinal inputs as well as segmental afferent influence (2002). When stimulated, propriospinals alone can produce locomotor activity in some preparations (Jordan and Schmidt, 2002). Redundancy of long propriospinal interneurons with supraspinal reticulospinal projections makes them an ideal compensatory link between diminished supraspinal drive and the CPG for locomotion after $\mathrm{SCl}$. One of the most important features of the CNS, and the primary reason the CNS is capable of adapting to injury or disease, is its interconnectivity and redundancy (Basso et al., 2002; Warraich and Kleim, 2010; Marsh et al., 2011). Interconnectivity supports functional reorganization while redundancy enhances neural integration of information to improve sensory and/or motor and cognitive processing (Warraich and Kleim, 2010). Rather than leaving unused structures that were previously used for one processing modality, but were then isolated because of some trauma in their original circuitry pathway, the CNS is adept at recruiting that area to perform a new, perhaps completely different function (Warraich and Kleim, 2010).

Spinal hemisection, location specific lesioning, and double lesioning studies have illustrated the functional redundancy and species specific function of the pathways that mediate locomotor control. After low thoracic hemisection, or with specific sectioning of VLF or DLF, cats and monkeys can walk overground with their hindlimbs, albeit with altered interlimb coupling (Rossignol et al., 1999). Rossignol and colleagues performed double lesion studies in which cats were subsequently spinalized (T13) after a few weeks of step training to optimize and stabilize the stepping pattern after an initial hemisection at T10 
(Barriere et al., 2008; Martinez and Rossignol, 2011). These cats were able to step at high speeds $(\leq 0.8 \mathrm{~m} / \mathrm{s})$ within 24 hours of the subsequent full transection. This finding indicates that step training following a lateral hemisection induced plasticity in the locomotor circuitry allowing it to operate better in the absence of input from supraspinal structures than it otherwise would have been able to. This study also showed that the circuitry mediating this learning is located caudal to the spinalization at T13. Courtine and colleagues used a double low thoracic lateral hemisection model in combination with electrochemical excitation that enables the CPG to be activated in way that increases responses to afferent cues (Courtine et al., 2008; Courtine et al., 2009; van den Brand et al., 2012), however the van den Brand et al. study in particular has caveats. Slawinska and colleagues point out that bipedal step training used by many researchers including the Courtine laboratory induces the animal to use it's head, forelimbs and trunk to shift its center of mass forward resulting in increased loading of the hindlimbs. This increased loading likely induces spinal stepping rather than truly "voluntary stepping," If voluntary stepping was achieved the authors should have included a measure of overground stepping, but this evaluation is not present (Slawinska et al., 2012). Furthermore, there are naturally occurring repair mechanisms such as collateral sprouting, synapse remodeling, and changes in neuronal properties that may enhance spontaneous recovery and plasticity but are not necessarily dependent on pairing with step training. Spontaneous sprouting after hemisection lesions in rats (Bareyre et al., 2004) and in the CST tract of monkey (Rosenzweig et al., 2010) is well studied. Hemisection studies 
show remarkable recovery but the staggered incomplete injuries are designed to take advantage of the redundant commissural pathways and endogenous sprouting in the spinal cord.

Clinically Relevant Spinal Cord Injury

While transection and double lesion studies lend great insight into the neural mechanisms responsible for training the CPG, these injuries do not represent the majority of $\mathrm{SCl}$ patients. Incomplete injury models are more relevant to the patient population than complete anatomical transection:

Contusion with characteristic cavity formation represent $49 \%$ of spinal cord injuries, laceration and massive compression are tied at $\sim 20 \%, 10 \%$ are solid cord injuries where no outright damage is visible until studied at the microstructure level (Grill 2005). There are rarely complete transections, some small amount of spared tissue usually remains (Bunge et al., 1993; Kakulas, 1999; Grill, 2005). The primary and secondary injury processes are different for each of these $\mathrm{SCl}$ classifications; therefore the type of injury model is of critical importance if our goal is to produce SCls that are clinically relevant.

Biomechanical characteristics of the spinal cord such as elasticity and viscosity determine how a trauma is distributed at a cellular level (Gruner, 1992). The velocity, force and displacement (compression) are factors in the severity of spinal cord injury since each affects white matter and grey matter damage differently. Higher velocity injuries produce more axon shearing while greater displacement and dwell time of compression produce more grey matter damage. Since white matter sparing is a direct correlate of functional recovery (Basso et 
al., 1995) an injury model that produces more or less axon shearing due to velocity is ideal if functional recovery due to the experimental intervention is a primary outcome measure. A contusion model highlights mechanisms of injury associated with white matter loss and velocity. Additionally, the internally pressurized 'tube' nature of the spinal cord and its associated vasculature produces cell dysfunction that spreads rostrally and caudally from the epicenter depending on the severity (Grill, 2005; Basso and Hansen, 2011). The contusion injury model is composed of weight drop device, $10 \mathrm{~g}$ rod, released from a specified height above the surface of the spinal cord and free falls with increasing velocity as it approaches the spinal cord (New York University Impactor, NYU) (Gruner, 1992). Compression injury models focus on tissue displacement/compression as the primary biomechanical production of $\mathrm{SCl}$ (Ohio State University, OSU) (Stokes, 1992; Stokes et al., 1992). For example, the Infinite Horizons $(\mathrm{IH})$ impactor uses a constant velocity and controllable force applied during the impact (Scheff et al., 2003). Fehlings and colleagues use a clip compression model that combines impact and compression injury characteristics and is applied to both ventral and dorsal aspects of the spinal cord (Fehlings et al., 1989). Laceration injuries can be used to selectively disrupt specific fiber tracts using microscalpels, microscissors, blades, or wire knife; however these tools often produce a contusion along with a laceration since they are not sharp enough to cut dura easily. An automated mechanized Vibraknife slices the tough dura more easily and produces an accurate reproducible laceration avoiding the contusion components of secondary injury (Zhang et al., 
2008). Laceration injuries are characterized by compromised dura and persistent ingrowth of connective tissue from muscle and surrounding soft tissue, and both lacerations and compressions produce a physical barrier of connective tissue scar between the severed ends (Zhang et al., 2004; Grill, 2005). A key factor of any injury model is that it generates a reproducible graded relationship of the severity of the injury to histological and behavioral outcome measures. Each of these models has been demonstrated to produce deficits in Basso, Beattie, Bresnahan Open Field Locomotor Scoring Scale (BBB), Basso Mouse Scale (BMS) and/or kinematic analysis of movement that correlate with increasing injury severity and loss of tissue at the injury epicenter (Basso et al., 1995; Scheff et al., 2003; Poon et al., 2007; Beare et al., 2009; Hill et al., 2009).

Activity-Based Training after Incomplete SCI

Battistuzzo recently systematically reviewed activity-based training (step training, swimming, voluntary exercise, and environmental enrichment) in animal models with lower thoracic incomplete SCI (Battistuzzo et al., 2012). After contusion or compression incomplete $\mathrm{SCI}$ rats and mice show remarkable spontaneous locomotor recovery (unlike after transection in which treadmill training with weight support is required) and are able to generate weightsupported stepping within 3-5 weeks post-injury as measured by BBB, BMS and/or kinematic analysis of hindlimbs (Fouad et al., 2000; Lankhorst et al., 2001; Thota et al., 2001; Multon et al., 2003; Van Meeteren et al., 2003; EngesserCesar et al., 2005; Koopmans et al., 2005; Magnuson et al., 2005b; Bolton et al., 2006; Erschbamer et al., 2006; Smith et al., 2006a; Fischer and Peduzzi, 2007; 
Carvalho et al., 2008; Heng and de Leon, 2008; Liu et al., 2008b; Siegenthaler et al., 2008; Robert et al., 2010; Alluin et al., 2011). Taken together, these studies show that voluntary exercise, enriched environment, step or swim training alone does not improve on the spontaneous locomotor recovery profile with sustained effects through the chronic phase. Out of these, a few studies have reported modest improvements in overground stepping beyond the first 2 weeks postinjury; however we consider these studies with the caveats that housing conditions (single, double, or socially housed), gender, body weight and age of animals may decrease in-cage activity before the activity-based locomotor training is applied. An increase in BBB scores was evident in the treadmill step training group after incomplete compression SCl (Multon et al., 2003). Epicenter histology from the Multon study found approximately $12 \%$ white matter sparing for both step trained and untrained groups, but the animals were housed individually. In our model using double housed adult female rats $(175-200 \mathrm{~g})$ the epicenters have a slightly lower percentage of white matter sparing, approximately $8-10 \%$, but BBB scores are higher than the untrained controls in studies with comparable contusion injury severities (Magnuson et al., 2005b; Smith et al., 2006a; Kuerzi et al., 2010). This indicates that the animals in the Multon study had lower in-cage activity because they were only singly housed. Therefore there was no ceiling effect and the benefits of step training were evident by a 2-3 point increase in BBB scores; however we question the impact of these increases since none of the rats went from only sweeping to actually frequent/consistent weight-supported stepping (BBB $\leq 10)$. 
Our center has already recognized that gender, species strain, age, weight and animal density per cage affect $\mathrm{SCl}$ outcome measures in a complex and interrelated manner (Burke et al., 2007). We consider double housing as beneficial for spontaneous locomotor recovery in untrained animals, presumably due to reduced stress and increased social interaction. When rats are housed singly (Multon et al., 2003; Engesser-Cesar et al., 2005; Stevens et al., 2006; Siegenthaler et al., 2008), or when male rats with heavy body weight (Carvalho et al., 2008; Siegenthaler et al., 2008) are used for locomotor training studies after incomplete $\mathrm{SCl}$, the benefits to overground stepping may appear to be more effective because locomotor activity as a whole is lower before the step training intervention. During in-cage activity animals are capable of hindlimb movement or stepping at any time during recovery. For example, rats have a lower center of gravity, can roll from side to side, drag their hindlimbs, freely move hindlimb joints through varied range of flexion and extension, and at some point begin to weight support and take steps just from moving around in their normal cages. Selftraining by rats after incomplete $\mathrm{SCl}$ is recognized in several publications (Fouad et al., 2000; Heng and de Leon, 2008; Maier et al., 2009; Fouad and Tetzlaff, 2011), and is described as a ceiling effect in our lab (Kuerzi et al., 2010). The purpose of the studies described herein is to address a component of the overall lab hypothesis, rats with T9 contusion injuries achieve maximal locomotor recovery by self-training in their cages, by testing whether immobilization (preventing in-cage activity/self-training) and stretch interventions alter the profile 
of functional locomotor recovery in our model of incomplete low thoracic contusion SCl.

The Issue of Translation

Unlike incompletely injured laboratory animals that move about in their cages soon after injury, the standard of care for SCl patients is early immobilization. A patient may have multiple traumas to the body in addition to CNS injury and may be in critical condition requiring first responders to perform emergency life saving procedures like stabilizing blood pressure, pulse and respiratory rate (Oyinbo, 2011). Increased survivability after SCI due to early surgical management including vertebral stabilization and decompression of the injury site has been the single most successful contribution to the field over the past several decades (Tator, 2006). As reviewed by O'Sullivan and Schmitz, beyond this acute phase of emergency care, immobilization and bed rest are deemed necessary to prevent further neurological impairment but also because paralysis is common (O'Sullivan and Schmitz, 2001). Patients with thoracic and lumbar fractions often need surgically placed internal fixation devices and external spinal orthoses for a minimum of three months. Following release from the trauma center, patients undergo widely varying kinds and amounts of physical and occupational therapy. Unfortunately insurance companies often dictate the duration of therapeutic physical interventions, and patients may not receive the necessary amount to achieve functional benefits (Harkema et al., 2011). During the acute period of immobilization, patients receive range-ofmotion (ROM) and muscle stretch therapy for both upper and lower extremities 
and for the trunk (O'Sullivan and Schmitz, 2001). Although early involvement in functional activities is advocated, the majority of patients are not able to engage in comprehensive locomotor rehabilitation regimen within the first few months after $\mathrm{SCl}$. Thus, when comparing experimental $\mathrm{SCl}$ in animal models with patients in clinical setting the locomotor and rehabilitation aspects are basically opposite: lab animals with incomplete injuries move about early after $\mathrm{SCl}$ and receive very little manual rehabilitation, while $\mathrm{SCI}$ patients are largely immobilized but manual therapies are standard of care.

Activity-based rehabilitation in the form of overground or BWST locomotor training (LT) was born of Wernig and colleagues' Laufband Therapy for SCl patients (Wernig and Muller, 1992; Wernig et al., 1995; Wernig et al., 1999), and has become one of the most widely accepted therapies translated from animal research for SCl patients (Warraich and Kleim, 2010; Marsh et al., 2011). LT rehabilitation strategies for patients are interpreted from motor control studies in the transected cat model, however the transection lesions of these models are not clinically relevant. Neither BWST nor overground LT provides the level of functional recovery that the pre-clinical transection animal studies were extrapolated to promise for $\mathrm{SCl}$ patients. Thus, there is a collective realization from researchers, working in both animal models and in clinical settings, that we do not yet have a comprehensive treatment strategy for $\mathrm{SCl}$ that produces reliable, robust locomotor recovery and is widely applicable to the majority of $\mathrm{SCl}$ patients. This dissertation work addresses two possible contributors to the inability to fully translate promising preclinical findings as they relate to 
neurorehabilitation after $\mathrm{SCl}$ : 1) Translation of activity-based rehabilitation strategies after $\mathrm{SCl}$ is difficult because interpretations are based on animal studies using models that do not sufficiently mimic the clinical situation, 2) Animal models of neurorehabilitation do not use the same standard of care that patients receive after neurotrauma. An analytical approach to translational neurorehabilitation reveals that animal models do not closely represent the patient condition after neurotrauma, and widely accepted standards of care are not always based on basic science/preclinical evidence.

Goals

The realization that significant locomotor recovery is achieved after incomplete $\mathrm{SCl}$ in the rat model but not by patients with what we believe are roughly equivalent injuries led us to develop an experimental model that includes aspects of acute patient care; immobilization and manual physical therapy. If the hindlimbs are immobilized then rats cannot experience in-cage activity (autotraining), which we believe is responsible for their significant locomotor recovery after low thoracic contusion injuries. In the first study, presented in Chapter 2 (Caudle et al., 2011), we tested whether hindlimb immobilization would alter the profile of locomotor recovery after mild T9 contusions. In the second study, presented in Chapter 3 , we sought to test whether the timing and duration of hindlimb immobilization would prevent the rapid and substantial gain of locomotor recovery that occurs after incomplete $\mathrm{SCl}$ in the rat model. The goal of the third study, presented in Chapter 4, was to further examine the detrimental effect of hindlimb muscle stretch in rats that are never wheelchair immobilized, as we 
found in Chapter 2, with a more severe T9 contusion. In all three studies we characterized a novel and feasible animal model of recovery after a clinically relevant $\mathrm{SCl}$ injury. Our model more closely mimics standard of care for $\mathrm{SCl}$ patients that shows both gain and loss of locomotor function that impact how we interpret studies on neurorehabilitation. 


\section{CHAPTER II}

\section{HINDLIMB IMMOBILIZATION IN A WHEELCHAIR ALTERS FUNCTIONAL RECOVERY FOLLOWING CONTUSIVE SPINAL CORD INJURY IN THE ADULT RAT}

The final, definitive version of Chapter II has been published in Neurorehabilitation and Neural Repair, 2011 Oct; 25(8): 729-39, by SAGE Publications Ltd., All rights reserved. (c)

\section{INTRODUCTION}

A potential impediment to the translation of activity-based rehabilitation following spinal cord injury (SCI) from animal models to patients is that the conditions of recovery in animal models do not parallel those of patients. Most patients are immobile over the first few weeks following injury, except for muscle stretch and passive joint range-of-motion (ROM) therapies (Beres-Jones et al., 2003; Dobkin et al., 2006). In contrast, animals used in experimental studies of $\mathrm{SCl}$ begin to move about in their cages within a few days or a week of injury. With a few exceptions (Roy et al., 1992; Hodgson et al., 1994; Smarick et al., 2007), the majority of animal studies do not mention the use of stretch or ROM therapies, yet these are widely accepted in clinical practice world wide (Harvey and Herbert, 2002; Harvey, 2008) and The Consortium for Spinal Cord Medicine prescribes that physical therapy begin within the first week post-injury and continue throughout the acute phase (Paralyzed Veterans of America 2008). A recent review of clinical trials of $\mathrm{SCI}$ rehabilitation found these therapies to be 
ineffective when compared to no intervention or conventional care (Harvey et al., 2002; Harvey et al., 2009). The effects of immobilization and/or stretching/ROM maneuvers in animal models of spinal cord injury remains largely unstudied.

Step training with weight support is a promising form of activity-based rehabilitation for $\mathrm{SCl}$ patients that is often used in conjunction with stretch/ROM therapies. The step training approach is deeply rooted in the elegant studies by Grillner, Rossignol, Edgerton and others showing that adult cats with complete spinal transections can be trained to perform quality hindlimb stepping via repeated exposure to the activity on a treadmill. Afferent input associated with phasic limb movements, loading and paw or foot contact is thought to train spinal circuitry below the injury (Wernig et al., 1995; Edgerton et al., 1997; Bouyer and Rossignol, 2003b). However, improvements in overground ambulation for patients participating in step training are variable and far less than expected based on the preclinical feline studies (Dietz et al., 1998; Dobkin, 2007; Nadeau et al., 2010). Similar to clinical studies, step training in rodent models of incomplete SCI has shown only modest, task-specific improvements in treadmill stepping that rarely lead to improvements in overground stepping (Basso et al., 1995; Fouad et al., 2000; Multon et al., 2003; Heng and de Leon, 2008), (de Leon personal communication 2009). In contrast to the clinical situation, however, adult rats with all but the most severe of incomplete injuries show remarkable spontaneous recovery leading some authors, including ourselves, to suggest that early in-cage activity provides sufficient amounts of appropriate afferent input to bring about substantial, even maximal, training that is difficult to 
improve upon using a variety of approaches including step training on the treadmill, stepping in shallow water or swimming (Fouad et al., 2000; Smith et al., 2006a; Heng and de Leon, 2008; Magnuson et al., 2009; Kuerzi et al., 2010;

Guertin et al., 2011; Singh et al., 2011).

The current study was undertaken to test the hypothesis that early in-cage activity is responsible for the dramatic functional improvements seen in adult rats with incomplete $\mathrm{SCl}$. We limited hindlimb activity and altered the pattern of activity-dependent afferent input by placing the animals in wheelchairs, 15-18 hours per day for 5 days a week starting at 4 days post-injury. In addition, we explored the influence of a daily stretch protocol on the profile of recovery in hindlimb immobilized animals and normally housed animals as controls. We found that periodic hindlimb immobilization in a wheelchair had a negative and lasting effect on functional recovery in rats with mild SCls, and that a daily passive stretch therapy had a lasting negative impact on recovery for normally housed $\mathrm{SCl}$ animals, but was not a factor for those experiencing hindlimb immobilization.

\section{MATERIALS AND METHODS}

Spinal Cord Injury and Experimental Design

Twenty-one female adult Sprague-Dawley rats (190-215g) were used for this study. All procedures involving experimental animals were performed according to the guidelines of the University of Louisville Institutional Animal Care and Use Committee. Animals were randomly assigned to three experimental groups: Wheelchair/Stretch (WC/SR), wheelchair/non-stretch (WC/non-SR), non- 
wheelchair/non-stretch (non-WC/SR) $n=5$ each, or non-wheelchair/non-stretch (non-WC/non-SR) controls $n=6$. Animals received contusion injuries at the $T 10$ spinal cord level as described previously (Smith et al., 2006a; Magnuson et al., 2009). Each animal was anesthetized with pentobarbital (55 mg/kg i.p.) and given prophylactic antibiotics (Gentamicin Sulfate $15 \mathrm{mg} / \mathrm{kg} \mathrm{sc}$.) prior to surgery. Body temperatures were maintained at $36-37^{\circ} \mathrm{C}$ throughout the procedure. $\mathrm{A}$ single level laminectomy was performed at the T9 vertebrae and clamps were applied to T8 and T10 spinous processes to stabilize the column. The Infinite Horizons Impactor (Precision Systems and Instrumentation, LLC [PSI]; Scheff, 2003) was used to deliver $200 \mathrm{kD}$ moderate contusion injuries at the T10 spinal cord level using a custom-made vertebral stabilization system. After injury, wounds were closed in layers using silk sutures with topical antibiotics applied to the incision. Starting on the day of surgery, post-injury day 0 , animals were housed two per cage in standard cages using Alpha Dry bedding with food and water provided ad libitum. All animals were housed in the same room with a 12hour dark cycle (6 pm to $6 \mathrm{am}$ ) and received post-operative care daily, including manual bladder expression as needed.

Figure 1 shows a timeline of the study and all assessments. On post-injury day 4, animals in the two wheelchair (WC) groups spent 15-18 hours per day (from approximately $4 \mathrm{pm}$ to $8 \mathrm{am}$ ), 5 days per week for 8 weeks (dashed bar along time axis) immobilized in a four-wheeled rat wheelchair (Figure 2). WC cage mates stayed together, paired, in large breeding cages while non-WC animals remained paired in standard cages. Rats in SR groups received the 
enhanced daily care including the stretch protocol 5 mornings per week, for 8 weeks, beginning the morning of post-injury day 5 . Both WC immobilization and SR interventions ended at 8 weeks; assessments continued until 16 weeks. Rat Wheelchair Design

The wheelchairs held the hindlimbs in a position that resembled the early hindlimb dragging phase of recovery following $\mathrm{SCl}$ (Figure 2A). Figure 2B shows a representative sample of kinematic analysis of hindlimb position, at rest, during WC immobilization. Hindlimbs were statically positioned with joint angles of approximately $88^{\circ}$ (hip), $45^{\circ}$ (knee), and $155^{\circ}$ (ankle) and were held in position using Velcro straps. Wheelchairs were constructed of a lightweight polycarbonate platform (6.5" long $\times 3.5^{\prime \prime}$ wide) stabilized by 4 polyvinyl chloride wheels $\left(1.25^{\prime \prime}\right.$ diameter). Velcro straps around the ribcage, lower abdomen and lower hindlimb above the ankles kept the rat in a stationary position. Dr. Scholl's Moleskin Plus adhesive padding lined the platform for comfort. An oval opening (2.0" length and $.75^{\prime \prime}$ wide) towards the rear of the platform allowed urine and fecal matter to drop through the wheelchair to the cage bottom. Wheelchair cages were fitted with a raised floor of wire mesh that allowed feces, urine and food debris to fall through to Alpha Dry bedding below. No animals in this study exhibited autophagia. Wheelchairs were washed daily and Chew Guard, Collasate, and/or metronidazole with New Skin paste (Zhang et al., 2001) was applied to deter chewing of the wheelchair and straps.

Daily Care and Stretch Protocol 
In addition to standard post-operative care, all animals received daily care 5 mornings per week for the first 8 weeks of study. Daily care consisted of washing with warm water and towel dry, and massage of the hindlimbs in a distal to proximal direction for pressure relief and circulation. In an effort to maintain skin integrity, WC animals underwent a thorough inspection immediately upon removal from the wheelchairs. Indentations in the skin under the ribcage and hip straps were common and usually resolved following washing and massage. Small abrasions occasionally developed due to the straps, but these also resolved following strap adjustment, diligent treatment with topical antibiotics and protection with gauze. All animals were weighed preoperatively and then weekly beginning at week 2 . The left side hindlimb tibialis anterior (TA), medial and lateral gastrocnemii (MG and LG, respectively) were harvested upon termination in order to assess muscle atrophy. MG and LG were weighed together.

Following daily care, animals in the SR groups received a 20-minute hindlimb stretch protocol, consisting of 2 sets of bilateral hindlimb manipulations that held each joint in maximally flexed and extended positions for thirty seconds each. Each joint was stretched as follows: ankle, knee, hip flexion and extension, and hip abduction and adduction. Handlers were trained to manipulate the hindlimbs using exact hand positions in order to isolate each joint (demonstration by W. Lee Smith, PT, Frazier Rehabilitation Institute, Louisville, KY). Each rat was loosely wrapped in a towel and held in a semi-supine position in one hand. Behaviors such as vocalization, or rapid movement of the hindlimbs and/or body were used as indicators of how tolerant the rat was to each position and resulted 
in adjustment of hand position so that the rat would lie still for the duration of the protocol. The SR protocol was performed in timed sessions, one handler per animal, such that all handlers could confirm hand positions in order to maintain consistent and standardized technique. Handlers documented each stretch session noting the side and joint order, observations about joint stiffness and any loss of range-of-motion. Side order and handler to rat pairings were randomized over the sessions.

Behavioral and Sensory Assessment

The BBB Open Field Locomotor Scale is a 0-21 point scale that evaluates hindlimb function while the animal behaves naturally in an open field ( $36^{\prime \prime}$ in diameter) for duration of 4 minutes (Basso et al., 1995). BBB evaluation was performed preoperatively, on post-injury day 4 , twice per week during the first 8 weeks of study and weekly thereafter. The animals were presented at random to the same two observers, both blinded to experimental groups, for all behavioral scoring.

The Louisville Swim Scale (LSS) is a 0-17 point scale developed in our laboratory to assess swimming performance following spinal cord injury (Smith et al., 2006a). Five categories of the scale are assessed for frequency of occurrence during swimming, dependence on forelimbs, hindlimb movement, hindlimb alternation, trunk stability and body angle. The LSS subscore accounts for the pattern generation abilities in the hindlimbs of the animal by excluding the trunk stability and body angle portions of the assessment. Maximum scoring for trunk rotation and body angle is 3 points each, therefore the LSS subscore has a 
range of $0-11$. The swimming pool is a Plexiglas $\AA$ chamber $\left(60^{\prime \prime}\right.$ length, $7^{\prime \prime}$ wide, and $12^{\prime \prime}$ deep). Swimming assessments were performed preoperatively, and then periodically from weeks $3-11$. The animals were briefly reintroduced to the water two days prior to each LSS assessment to decrease any potential stress response.

Sensory testing was performed at single time points during weeks 3 and 4 to determine if hindlimb allodynia or somatosensory hypersensitivity could be contributors to the severe deficits in stepping for WC animals. Animals were arranged one in each of six partitions and allowed to acclimate in each apparatus for 30 min prior to testing. Both hindlimb paws were sampled five times, with a minimum of two minutes between consecutive samples. The von Frey filament testing surface was a metal grid with small holes (3/8" diameter) through which a narrow plastic cone shaped filament could be applied directly onto the plantar surface of the paw. The force (grams) required to elicit a withdrawal response (or induced the animal to attend to the paw) was recorded for each test (Berrocal 2007). The surface for the Hargreaves' test was a clear Plexiglas pre-warmed to $34^{\circ} \mathrm{C}$, and the apparatus was set at $30 \%$ intensity with a cutoff time of $20 \mathrm{sec}$. to avoid tissue damage. The heat source was focused on the plantar surface until the paw withdrew from the thermal stimulus; the amount of time to withdraw the paw is reported in seconds (Berrocal 2007). The von Frey test for mechanical allodynia showed no significant differences between groups at week 3 (WC/SR $11.3 \mathrm{~g} \pm 3.13, \mathrm{WC} /$ non-SR $10.3 \mathrm{~g} \pm 2.08$, non-WC/SR $9.0 \mathrm{~g} \pm 3.12$, non-WC/nonSR controls $11.5 \mathrm{~g} \pm 2.90)$. At week 4 , the Hargreaves' test for thermal 
hyperalgesia revealed no group differences (WC/SR $45.4 \mathrm{~s} \pm 12.39, \mathrm{WC} / \mathrm{non}-\mathrm{SR}$

$47.4 \mathrm{~s} \pm 12.06$, non-WC/SR $43.1 \mathrm{~s} \pm 8.37$, non-WC/non-SR controls $40.5 \mathrm{~s} \pm$

10.13).

Kinematic Assessment of Stepping and Swimming

To quantitatively assess hindlimb movement during walking and

swimming, we performed kinematic analysis at various time points throughout the study as previously described (Magnuson et al., 2009; Kuerzi et al., 2010).

Overground stepping was assessed using a two camera, three-dimensional (3D) kinematic analysis performed pre-injury and at 6 and 11 weeks. Quantification of cycle characteristics represents bilateral hindlimb movements averaged for both sides with a minimum of 5 cycles sampled per side. Hindlimb movement was recorded with two Basler $602 \mathrm{f}$ high-resolution digital cameras running at $60 \mathrm{~Hz}$ connected to a PC using the video software DVR Explorer (Advanced Digital Vision, Natick, MA). Digital AVI files from both cameras were opened in MaxTraq3D (Innovision Systems, Columbiaville, MI) where sharpie marks overlying the iliac crest $(\mathrm{I})$, hip $(\mathrm{H})$, ankle $(\mathrm{A})$ and toe $(\mathrm{T})$ were identified in a semiautomated fashion on each frame. The two dimensional files were digitally combined to estimate a 3D limb position for each frame from which all stick figures and angle excursion data is derived in Excel or MaxMate (Innovision Systems).

In-vivo Micro-CT Scanning of Bone

Animals that did not receive SR protocol (WC/non-SR and non-WC/non-

SR) underwent micro-CT scanning of distal femora at 6 and 13 weeks. All micro- 
CT scanning was performed as described previously (Voor et al., 2008) using a custom scanner (150/225-Ffi-HR-CT, BIR, Lincolnshire, IL) that includes a 225 KV X-ray source with a focal spot size of $5 \mu \mathrm{m}$ and an image intensifier with a 1024 x 1024 pixel digital camera (ACTIS, BIR, Lincolnshire, IL). The basic X-ray power settings were $86 \mathrm{kV}$ and $110 \mu \mathrm{A}$. Briefly, animals were maintained under isoflurane anesthesia and ventilated (VSA-2100, VetLand, Louisville, KY; Model 2000 ventilator, Hallowell, Pittsfield, MA). Each rat was positioned with the left hindlimb flexed and the right hindlimb extended into the lower column of the animal manipulator. Scans represent a full $360^{\circ}$-plus-fan-angle rotation, with 1000 projections per revolution, covering a $3 \mathrm{~mm}$ distance below the knee joint. Two complete rotations covering 214 slices were performed for each scan; the total time in the micro-CT scanner was approximately 30 minutes; total X-ray exposure time was approximately 12 minutes. A custom MATLAB program (v6.5, The Mathworks, Matick, MA) was used to isolate the compact bone from the less dense cancellous or trabecular bone, and to calculate the bone volume fraction (BV/TV) $(\mathrm{Xu}, 2008)$. Three-dimensional images were reconstructed using the voxel analysis software VGStudio Max (v1.2.1, Volume Graphics, Heidelberg, Germany). Data is presented in Figure 5 as means $\pm S D$ based on contracture groupings within the WC/non-SR group; no-contracture, $n=2$; contracture, $n=3$; and non-WC/non-SR controls, $\mathrm{n}=6$.

Electrophysiological Assessment

Some WC animals developed ankle pathologies that resulted in a severe loss of range-of-motion. To assess the tone of, and estimate spasticity in an 
ankle extensor, we performed simultaneous bilateral electromyographic (EMG) recordings of lateral gastrocnemius muscles (LG) at rest and during tail and paw pinches for all animals. Each rat was positioned, unanesthetized, on a raised wooden platform so that the hindlimbs were able to straddle either side of a narrowed strip between the legs, allowing the legs to hang down without contacting a surface. A cloth stockinette was used to securely hold the animal in place with the hindlimbs, head and tail outside of the cloth, as described previously (Magnuson et al., 2005b). A small puncture was made in the shaved skin overlying the belly of the LG using an 18-gauge needle tip. A fine wire intramuscular electrode was positioned into the tip of a 23-gauge needle, fed through the cutaneous puncture, and inserted into the muscle. The needle tip was removed and electrode placement was confirmed by a manual palpation during a passive range-of-motion maneuver in cases where the ankle was capable of flexion. Reference electrodes were placed subcutaneously on the medial side of the Achilles' tendon and the ground electrode was placed at the base of the tail. The fine wire electrodes were connected to Al 405 head stages and a CyberAmp 380 (Axon Instruments). Data were acquired in Axoscope running on a Dell PC with sampling rate of $1 \mathrm{KHz}$.

Tail and paw pinch involved closing a rubberized paper clamp 1" from the base of the tail, and onto the plantar and dorsal aspects of the paw for approximately $1 \mathrm{sec}$, repeated 5 times over a period of 3-5 minutes. Recordings were filtered and rectified for analysis in Axograph 4.0 on a Macintosh G4 computer to determine the timing of burst onset and offset. The percent bilateral 
co-contraction of LG muscles during tail and paw pinch was calculated as a surrogate indicator of spasticity (Boorman et al., 1996; Morita et al., 2001). The duration of co-activity was divided by the total duration of activity (either muscle) per set of bursts and converted to a percentage.

Histology of Sciatic Nerve and Spinal Cord Injury Epicenter All animals were euthanized at the end of week 16 and perfused transcardially with $4 \%$ paraformaldehyde and calcium free tyrodes solution. The entire length of spinal cord was removed for histological analysis, and sciatic nerves from each left hindlimb were harvested for analysis of cross-sectional area. Blunt dissection of the biceps femoris exposed a portion of the sciatic nerve, which was dissected out from approximately $2 \mathrm{~mm}$ distal to the tibialfibular bifurcation upward to the inferior piriformis muscle, approximately $20 \mathrm{~mm}$ in total length. Adjacent sets of spinal cord sections were stained for white (eriochrome cyanine) or gray matter (cresyl echt violet for Nissl substance) and processed for spared white matter at the epicenter, damaged gray matter and cavity volume as previously described (Hadi et al., 2000; Magnuson et al., 2005b). Spinal cord and sciatic nerves were post-fixed overnight, followed by cryoprotection in $30 \%$ sucrose. Tissue was blocked into freezing medium, then cut on a cryostat at $40 \mu \mathrm{m}$ (five sets, each with every $5^{\text {th }}$ section) and mounted onto glass slides. Briefly, every fifth section from the epicenter was photographed with a SPOT digital camera (Medical Diagnostics) attached to a Macintosh computer and the total area of white matter, gray matter (with Nissl stained cell bodies), injured gray matter (punctate appearance with gliosis and lacking 
neuronal cell bodies), cavity and total area were traced using a Wacom Intuos (Vancouver, WA) drawing tablet. Traced images were opened in Image J (NIH) and cross sectional areas were calculated for each tissue region. The injury epicenter was determined as the section with the least spared white matter as identified by positive eriochrome cyanine stain and sampling was extended in the rostral and caudal direction until the total area had reached a plateau. The total volume of cavitation and injured gray matter across the extent of the lesion was calculated by multiplying the area of adjacent sections by the distance $(\mu \mathrm{m})$ between sampled sections. Analysis of the cross-sectional area of sciatic nerves included every fifth section proximal to the bifurcation. Sections were stained with cresyl violet and processed in the same manner as described for the spinal cord.

Statistical Analysis

All data are presented as group means with standard deviations $( \pm S D)$.

Outcome measures were analyzed using repeated measures analysis of variance (ANOVA) with groups as a factor, or one-way ANOVA and were followed by Tukey's HSD or Bonferroni post hoc $t$-tests where appropriate. Differences between groups were considered statistically significant for $p$ values of at least ps.05. The binomial proportions test was used to determine the validity of regrouping animals based on presence or absence of ankle contracture (contracture and no-contracture, respectively) and to determine differences in the number of animals per group with ankle contractures. Proportions were checked to ensure the sample size was not too small nor the proportions too extreme for comparison (Siegel and Castellan, 1988). No corrections to the proportions were 
required. All data was sorted and assessed for significant differences as follows: 1) based on the original experimental design WC/SR, WC/non-SR, non-WC/SR, non-WC/non-SR controls; and in cases where the data indicate a bimodal distribution, 2) based on contracture or no-contracture.

\section{RESULTS}

\section{Behavioral Assessment}

BBB assessments were performed twice weekly for the first 8 weeks during which wheelchair immobilization (WC) and/or hindlimb stretch (SR) were interventions (Figure 2C and D, red bar along time axis), and then weekly for the remainder of the 16 week study. Animals were taken out of the wheelchairs, underwent daily care with or without SR, and were placed into standard housing, 2 per cage, for at least 4 hours prior to any assessments. All BBB assessments for both WC groups are compared to control animals (Figure $2 C, W C / S R n=5$, $W C / n o n-S R n=5$ vs. non- $W C /$ non-SR $n=6)$, and non-WC animals that received SR are compared to controls separately (Figure $2 C$, non-WC/SR $n=5$ vs. nonWC/non-SR $n=6)$. The group averages were similar for all 4 groups in two aspects: (1) At post-injury day 4 all animals, as expected, were dragging their hindlimbs with no weight support and had varying degrees of hindlimb joint movement; (2) at post-injury day 7, most animals had some degree of weight support and plantar stepping. Figure $2 \mathrm{C}$ shows a profile of recovery that was significantly different for both WC groups as compared to controls beginning at week 2 through the remainder of the 16 week study $( \pm S D, n=5 / 6, p \leq .05)$. Loss of function for WC groups became evident at week 2 and the decline in BBB scores 
continued until weeks 4-5. At week 4, WC animals exhibited slight to extensive movement of hindlimb joints and were consistently dragging their hindlimbs (WC/SR group average $B B B=1.9 \pm 1.9, W C /$ non-SR=3.9 \pm 2.3 ); in contrast, the control group had recovered consistent weight-supported plantar stepping with consistent coordination (non-WC/non-SR, BBB=14.0 \pm 2.4 ).

The wheelchair immobilization and stretch protocol ended at week 8 , and statistically significant deficits in BBB scores remained for both WC groups (WC/SR $5.5 \pm 2.1, W C /$ non-SR $4.7 \pm 3.3$ ), as compared to control animals whose scores had reached a plateau (non-WC/non-SR $17.8 \pm 1.7$ ). WC groups regained some function between weeks 6 and 10, however group averages indicate that the inability to achieve weight support with consistent plantar stepping persisted for the duration of the study (BBB $\leq 10$ denoted by the dashed line in Figure $2 C$ ). These data represent a substantial effect of intermittent WC immobilization, in animals that could move around in their standard cages for approximately 8 hours per weekday and continuously (approximately 56 hours) each weekend.

Any effect of the daily SR protocol on WC animals was either minimal and/or was rendered undetectable by the negative influence of wheelchair immobilization. We anticipated the need for circulatory and muscle maintenance for WC animals, and the SR protocol was included as an effort to alleviate any pathologies associated with hindlimb immobilization. Surprisingly, the BBB scores of the wheelchair/SR animals $(n=5)$ suggest they were detrimentally affected as compared to controls (Figure 2C). Beginning at week 6, the group mean BBB scores were significantly lower (non-WC/SR $12.4 \pm 2.2$ ) than the non- 
SR control group ( $\pm S D, n=5 / 6, p \leq .05)$. The dotted horizontal line, $B B B=14$, indicates consistent forelimb-hindlimb coordination; although the standard deviation for this group is high, $\sim 3$ points, these data show that receiving a 20 minute protocol of hindlimb stretch therapy daily produced a group average BBB score indicating an inability to achieve consistent coordination.

Prevalence of Ankle Contracture

The profile of recovery in BBB scores for WC groups in Figure $2 \mathrm{C}$ can be characterized as short-term loss of function during weeks $2-4$ followed by regain of function in weeks $8-10$. It should be noted that all behavioral scoring took place in the afternoons at least 4 hours after WC animals were removed from their wheelchairs and placed into standard housing (2 per cage). Nonetheless, some of the dramatic loss of function for WC animals may have been due to transient stiffness or loss of passive ROM (developing contractures). Indeed, beginning at post-injury weeks $4-5$, some WC animals experienced pathological changes to the hindlimb ankle joint resulting in an inability to move the joint regardless of neurological function (Moriyama et al., 2006; Moriyama et al., 2007). The average terminal BBB score for a leg with an ankle contracture was 7 , and necessarily prevented that leg from achieving plantar placement. Assuming contracture of one or the other ankle is an independent process, there were a significantly higher proportion (ps.001) of ankle contractures on either side for WC animals ( $n=9$ out of 20 ankles total) versus non-WC ( $n=0$ out of 22 ankles). Importantly, inclusion in the SR protocol group made no difference in the occurrence rate of ankle contracture in WC animals (WC/SR $n=4$ out of 10 ankles 
and $W C / n o n-S R ~ n=5$ out of 10 ankles). Since the SR protocol had no apparent effect on promoting or preventing contracture, each of the WC animals $(n=10)$ was reassigned based on the presence/absence of contracture: WC/contracture $n=4, W C /$ no-contracture $n=3$, and $n=3$ animals had contracture unilaterally only. Using a binomial proportion test, we compared these new groups with controls and re-assessed the data set.

Figure $2 \mathrm{D}$ represents the same data as shown in Figure $2 \mathrm{C}$, but is regrouped based on presence (WC/contracture, $n=3)$ or absence (WC/nocontracture, $n=4$ ) of ankle contractures in the WC animals. The profile of recovery during weeks $2-4$, indicating a loss of function for WC animals, remains similar to Figure 2C. WC animals, with and without ankle contractures, have significantly lower scores as compared to controls beginning at week 2.5 and persisting to the end of the study. Weeks $10-16$ reveal significantly different degrees of functional recovery between animals that had no contractures versus those that did develop chronic ankle pathology. During weeks $10-16$, WC animals with no discernable ankle pathology recovered frequent weight-supported stepping lacking coordination (WC/no-contracture highest mean BBB score 11.6 \pm .75 , week 14) whereas animals with ankle contracture could not achieve extensive movement of all three hindlimb joints nor plantar placement (WC/contracture highest mean score $7.3 \pm .58$, week 15 ).

Kinematic Assessment of Stepping

For stepping, group 3D kinematic excursion data was quantified for the hip, knee and ankle (Figure 3A). Group averages were compared at weeks 6 and 
11. At week 6, WC animals showed significantly reduced mean excursions for all three joints as compared to non-WC animals (Figure 3A). Hip and knee positions were relatively flexed ( 70 and $40^{\circ}$ respectively) while the ankles were extended (140-160 ; Figure 3B and D). By week 11, both WC groups regained hip excursions that were not different from controls and also regained some knee excursion, which remained significantly different from non-WC animals by having a lower trough value (flexion; Figure $3 \mathrm{C}$ and $\mathrm{E}$ ). Mean ankle excursions at week 11 were significantly different for the two WC groups compared to both non-WC groups, and also between WC groups (WC groups contracture/no contracture; Figure 3A). By week 11, WC animals without contractures had recovered sufficient ROM about the ankle to achieve weight-supported, plantar stepping (Figure $3 \mathrm{~A}$ and $\mathrm{C}$ ), while animals with ankle contractures had very low excursion ranges reflecting their very limited ankle ROM. There were no differences between non-WC groups with or without SR at any time points indicating that deficits observed using the BBB scale were in interlimb coordination and not in joint excursions during stepping.

Swimming Assessment

Swimming was used as an assessment of locomotion that does not require the ability to support body weight. Swimming ability was assessed using the Louisville Swim Scale (LSS) with supplemental cutaneous feedback and the subscore was calculated by excluding the 6 points allotted for body position and trunk stability (Smith et al., 2006b). Like the other indicators of locomotor function, both WC groups exhibited significantly lower scores for swimming than both non-WC groups during weeks 3, 5, 7 and 11 (Figure 4A). As characterized 
by the LSS subscore (Figure 4B), both WC groups had significant deficits in hindlimb movement when compared to the non-WC groups both during and after WC immobilization and/or SR interventions (weeks 3, 5, 7, 11 and 13). By week 9, one week after the WC and/or SR intervention had stopped, the WC animals with no contractures had gained $\sim 3$ points on the LSS scale and only animals that had contractures had significantly lower scores than controls for the remaining weeks. These data indicate that the greatest amount of recovery for the WC animals without contractures occurred in their ability to generate the cyclic hindlimb pattern of swimming.

These observations from the LSS Scale were supported by our 2D kinematic assessment of swimming based on angle-angle plots constructed on a stroke-by-stroke basis for the iliac crest-hip-ankle (IHA) and hip-ankle-toe (HAT) angles. Mean areas $( \pm S D)$ and representative examples of IHA-HAT angle-angle plots from the terminal time point (Figure $4 \mathrm{C}$ and $\mathrm{D}$ ) illustrate that all three groups without ankle contractures recovered the ability to generate coordinated hindlimb kicking with similar plot areas. Mean centroid positions (Figure 4E) illustrate that hindlimb restriction in the wheelchairs did alter the mean HAT position during kicking $\left(160^{\circ}\right.$ significantly extended compared to non-WC groups) while the presence of an ankle contracture resulted in a mean HAT angle of greater than $200^{\circ}$ (Figure 4E). Interestingly, all four groups were capable of generating toe velocities (relative to the hip) greater than $40 \mathrm{~mm} / \mathrm{sec}$.; animals in the WC/con group accomplished this despite the lack of ankle ROM using only their hips and knees (Figure 4F). 
No significant differences in LSS Score or LSS Subscore were found for non-WC/non-SR and non-WC/SR animals despite the significant differences previously identified in overground stepping (Figure 2). Thus, we calculated the BBB subscore for the three groups without ankle contractures and found that the non-WC/non-SR control group had significantly better subscores than the nonWC/SR and WC/no-con groups (Figure 4G). The BBB subscore, which focuses on fine aspects of stepping, could not be calculated for animals with ankle contractures. Thus, the SR protocol appears to negatively influence the recovery of stepping more strongly than the recovery of swimming.

In-vivo Micro-CT Scanning of Hindlimb Bone

We used in-vivo micro-CT scanning at 6 and 13 weeks to assess bone loss and cancellous bone turnover in the proximal tibiae of WC and non-WC animals that did not receive the SR protocol (Figure 5A - C). We assessed only non-SR animals and separated out animals with contractures, which reduced our sample size of WC/no-con animals to 2 , limiting our statistical power. There was a trend towards decreased Bone Volume/Total Volume ratio at 6 weeks, which persisted to 13 weeks for WC/no-con animals when compared to WC/con and non-WC/non-SR animals (Figure 5A). WC immobilized animals that developed contracture of the ankle, $n=3$, had bone volumes very similar to that of the control group, $n=6$. Representative scans shown in Figure $5 B$ and $C$ reveal visual differences in the density of the trabecular structure along the lateral condyle and shaft (arrows) between animals. These findings suggest that our model may be 
sufficient to induce bone remodeling, and daily in-cage movement may be related to preservation of bone structure.

Body, Muscle Weight and Sciatic Assessment

We assessed body weight on a regular basis, and left hindlimb muscles

TA and MG/LG, terminally, as indicators of possible negative effects of intermittent immobilization and unloading (Figure 6). WC immobilized animals lost weight during the first few weeks after injury and then gained weight thereafter. WC groups gained weight at a similar rate as non-WC groups however they were significantly lighter throughout the 8 weeks of WC and/or SR interventions, but no differences existed for weeks 8 through 16 (data not shown). We used the left side hindlimb muscle wet weight, rather than the right, based on the criteria that the ankles have no apparent lack of ROM and therefore a greater probability of having been plantar loaded. This criteria was true for $n=7$ out of 10 animals ( $n=4$ WC/no-contracture bilaterally, plus $n=3$ contracture on the right ankle only), and the animals with bilateral contracture ( $n=3 \mathrm{WC} /$ contracture) would have had equal compensation on both sides; we therefore avoided disproportionate representation of a limb that had greater disuse and less loading. The MG/LG (ankle extensor) muscle wet weights were $10 \%$ less for both WC groups at the terminal time point and no differences were found for the TA (ankle flexor; Figure 6A, ps.05). Cross-sectional area of left side sciatic nerve was significantly lower for WC animals that did not receive the SR protocol as compared to non-WC animals that received SR intervention only (Figure 6B). Interestingly, these data indicate a possible positive effect of SR for fiber area. 
EMG Recordings of Gastrocnemius Muscle

At the end of WC and/or SR interventions (week 8), we assessed all

animals for co-contraction (as a surrogate of spasticity) of right and left

gastrocnemius muscles in an effort to identify possible contributions to the severe loss of ROM about the ankle (Trudel et al., 2008) (Figure 7). Electrophysiological assessment consisted of several 1-minute at-rest recordings, with a series of brief tail or paw pinches that consistently elicited visually obvious hindlimb responses. Figure $5 \mathrm{~F}$ shows the percentage of co-contraction of bilateral LG muscles during tail and paw pinches revealing that WC animals had significantly greater co-contraction than non-WC animals for tail pinch (WC $71.2 \% \pm 6.87$ versus non-WC $55.8 \% \pm 16.66, n=10 / 11$ respectively), but no significant differences were found in the paw pinch condition. The inset lists the rank order of percent co-contraction found among all 4 original groups (Figure 7). WC animals that did not receive SR had the highest percent of co-contraction (72\%) while the control animals had the lowest percent $(47 \%)$, and these differences were found to be significant $(p \leq .05)$.

Histological Assessment

Using a one-way ANOVA, no significant differences were found in the percent of spared white matter (cross sectional area), gray matter loss or cavity volume $\left(\mathrm{mm}^{3}\right)$ at the epicenter among any groups (Table 1). The amount of spared white matter at the epicenter $(30 \%)$ is consistent with the functional recovery achieved by the control group as measured by the BBB Scale $(\sim 18)$ (Magnuson et al., 2005a). BBB scores of $\sim 10$ at week 1 and terminal scores of $\sim 18$ for injured controls are higher than expected with a $200 \mathrm{kD}$ contusion injury 
using the Infinite Horizons system. We attribute the relatively mild injuries to the custom-made system we used to stabilize the vertebrae, which was novel for us at the time. Nonetheless, all the animals were injured in a consistent manner.

\section{DISCUSSION}

It has been more than three decades since Sten Grillner and his talented trainees began referring to "the central generation of locomotion" while studying the low spinal cat model (Grillner and Zangger, 1979; Barbeau et al., 1999a). Since then, this model of acute transection has been used to characterize the capabilities of the lumbar spinal cord and the principles underlying locomotor training on the treadmill. The work of many laboratories including those of Edgerton, Rossignol and Barbeau have identified several issues including phasic limb loading, cutaneous paw contact and load progression as critical to training (Harkema et al., 1997; Barbeau et al., 1999a; Bouyer and Rossignol, 2003b; Cote and Gossard, 2004; Timoszyk et al., 2005; Cha et al., 2007; Frigon and Rossignol, 2008). In addition, training is thought to be task-specific in that improvements are largely limited to the trained task (Edgerton et al., 1997; de Leon et al., 1998; Smith et al., 2006a; Bigbee et al., 2007).

Translating these key principles to the rodent and human models, however, has not been straightforward. Inherent to these three models, human, cat and rat, are a number of critical differences, as reviewed by Majczynski and Slawinska (2007), which may hinder translation and complicate the interpretation of experimental results. For example, most rodent studies use mid-thoracic 
transections/contusions or incomplete lacerations, and acute or sub-acute training. In contrast, most clinical studies involve chronic incomplete or dyscomplete cervical or high-thoracic contusions. These three models also have very different locomotor characteristics: bipedal vs. quadrupedal, high vs. low vs. very low center of gravity. The characteristics of balance, trunk stability and posture, and the consequences of errors in stepping vary considerably and dramatically influence the capabilities of each species during the critical acute and sub-acute post-injury time period. Rodents are very active within days of injury while patients are largely immobile for several weeks or months, experiencing little more than transfer, stretching and passive range-of-motion therapies during this period. Apart from the substantial studies conducted by Dobkin and colleagues, rarely will patients enter an activity based rehabilitation setting before 3-6 months post-injury (Dobkin et al., 2006; Dobkin, 2007). Importantly, step training of spinal cord injured rodents consistently demonstrates task-specific changes in hindlimb function, but rarely achieves frank improvements in overground stepping. Fouad and colleagues used daily treadmill training in an over-hemisection rat model and were unable to uncover improvements in overground stepping using the BBB Scale and kinematics as outcomes (2000). As also alluded to earlier by de Leon et al. (1998), Fouad concluded that spontaneous recovery resulting from self-training was substantial and that treadmill training provided no added benefit (2000). Subsequently, Multon et al. (2003) used manually assisted treadmill training of rats with T7 microballoon compression injuries to demonstrate that small improvements in 
BBB Scores could be achieved. Notably, these animals were housed individually, without enrichment. Invariably, studies using rats with all but the most severe incomplete injuries report substantial improvements in overground locomotion over the first few weeks post-injury (Fouad et al., 2000; Basso et al., 2002;

Schucht et al., 2002; Multon et al., 2003; Magnuson et al., 2005a; Heng and de Leon, 2008). Similar reports for cats following thoracic contusion or incomplete laceration injuries are limited (Gorska et al., 1993) with the majority of studies being focused on task-specific improvements of limb function on the treadmill.

Taken together, these observations suggest that the in-cage activity exhibited by rats with acute incomplete SCls should be viewed as cumulative step training. Therefore, we hypothesized that incompletely injured rats maximally train themselves within the first few weeks after injury. To test this hypothesis we designed a 4-wheeled cart or wheelchair that dramatically restricts hindlimb movement by holding the limbs in an extended-drag position, and alters the normal pattern of afferent input while still permitting good in-cage mobility. Anticipating that hindlimb immobilization might induce pathologies of muscles and joints, we also developed a stretching protocol based on standard of care clinical physical therapy (Harvey et al., 2009). We found that neither intermittent hindlimb immobilization (WC) nor stretch (SR) initiated at 4 days post-injury, prior to the resolution of spinal shock, had any influence on the dramatic early increase in hindlimb function. Both SR and non-SR, WC and non-WC groups had similar BBB scores at 7 days post-injury $(10.6 \pm 1.43$ and $11.3 \pm 1.85$, respectively), suggesting that this phase of recovery is likely not dependent on 
activity or patterns of afferent input. Thereafter, WC (SR and non-SR) animals experienced a dramatic loss of hindlimb function leaving them unable to generate weight-supporting plantar steps by 3 weeks post-injury. Poor hindlimb function assessed using the BBB Scale and kinematically, persisted for the 8 week duration of WC immobilization. When returned to standard housing at 8 weeks post-injury function rebounded somewhat during weeks $8-10$, but remained significantly below the non-WC/non-SR control group out to week 16 suggesting that the spinal cord circuitry was not as responsive to in-cage training beyond 8 weeks post-injury. Both WC/SR and WC/non-SR groups also developed ankle pathologies at a $40-50 \%$ rate that dramatically limited ROM. Only modest decreases in muscle weights were observed for the LG and MG only (not the TA) and the WC/SR group experienced loss similar to the WC/non-SR group. The SR protocol did appear to ameliorate a decrease in Bone Volume Ratio seen in the WC/non-SR group, however our group sizes were too small to achieve significance with this measure. Importantly, when the animals with ankle pathologies were excluded, a significant difference in the BBB scores and kinematic measures persisted showing that WC immobilization had a dramatic and lasting negative influence on locomotor function.

Conceptually, there are interesting parallels between the current work, the literature discussed above and the work of Maier et al. (2008) using a rat model of unilateral corticospinal tract injury. Maier and colleagues showed that constraining the use of one limb, thus forcing the use of the impaired limb, lead to dramatic improvements in function that were correlated to increased contralateral 
collateralization of corticospinal fibers in the cervical gray matter. When not forced to use the forelimb on the injured side, it remains immobile and the longterm functional (and anatomical) consequences of dies-use are dramatic.

In contrast to the anticipated loss of function brought about by WC hindlimb immobilization, we also observed a significant and surprising difference between the SR and non-SR groups of non-WC animals indicating that our stretch protocol was having a detrimental effect on hindlimb function. The differences between groups developed somewhat gradually between weeks 2 and 5 suggesting that the SR protocol was bringing about a functional plateau earlier than observed in the non-SR control animals. Despite stretching/ROM being a standard of care practiced clinically, systematic literature reviews have suggested that these approaches have mixed benefits or the data appears inconclusive at best (Harvey et al., 2009). While many dubious explanations may be put forward for our observations, the most feasible is that our stretching protocol was activating barrages of afferent activity that disrupted lumbosacral circuitry and plasticity. The Grau laboratory, using an instrumental learning paradigm has demonstrated that randomly applied (uncontrollable) noxious inputs can disrupt spinal cord plasticity associated with both the short-term establishment phase and longer term maintenance phase of instrumental learning in the fully transected rat preparation (Crown et al., 2002; Patton et al., 2004). Importantly, they also uncovered evidence that only six minutes (cumulative) of uncontrollable noxious input (tail shock) can negatively alter the normal profile of locomotor recovery in a contusion model of spinal cord injury 
(Grau et al., 2004). Thus, we posit that our SR protocol, acting as an uncontrollable input, had a negative influence on the process of plasticity that leads or allows in-cage activity-dependent functional recovery. The non-WC/SR animals in our study were high functioning; they exhibited consistent weightsupported stepping as measured by the BBB and were not different from controls when assessed kinematically while moving freely overground and swimming, indicating the stretch protocol was not debilitating. The clinical implications of these observations are significant and future work should address the utility of stretch-based therapies for avoidance of peripheral pathologies versus the potential for negative long-term consequences on spinal learning and locomotor training.

WC immobilized animals also exhibited higher levels of antagonist muscle co-contraction in response to tail pinch, as a surrogate measure of spasticity, compared to non-WC animals. In addition, we routinely observed bouts of bilateral hindlimb spasms during wheelchair immobilization, which were not quantified. The combination of ankle contractures and spasticity suggests that the immobilization was not just preventing movement-related afferent input, but was possibly resulting in uncontrollable, noxious afferent input. Thus, while immobilization or inactivity may prevent optimal in-cage training, it remains to be determined how an absence or lack of activity-dependent afferent input compares to an abundance of activity-independent (uncontrollable), possibly noxious input to influence functional recovery (Grau et al., 2004). 
Recently, we discovered that providing $50-60 \%$ body weight support with $5 \mathrm{~cm}$ of water allows young adult female Sprague-Dawley rats with moderatelysevere $(25 \mathrm{~g}-\mathrm{cm})$ T9 contusion injuries to generate high-quality plantar hindlimb stepping with near-normal kinematics as early as 1 week post-injury in the absence of an applied training strategy (Kuerzi et al., 2010). In an elegant piece of work, Courtine and colleagues (Courtine et al., 2009) showed in fully transected rats that a combination of serotonergic agonists, epidural stimulation and weight support allowed near-normal stepping (albeit bipedal) to be expressed at 1 week post-injury. In the current study, nightly WC immobilization on days 4,5 and 6 post-injury, as spinal shock was resolving, had no influence on the day 7 BBB scores. Taken together, these findings suggest that a robust ability to generate a stepping pattern is present immediately post-injury, and that this capacity is not dependent on an acute re-training phenomenon. Importantly, however, in our shallow-water stepping model, we found that the acute stepping capacity was not maintained in the absence of continued exposure to the activity ("training"). This is in good agreement with the work of Courtine et al. (2009), described above, who found that excellent patterns induced at 1 week (with the "full combination" treatment) were not present at 9 weeks in animals that did not receive treadmill training in the intervening period. Thus, it follows that hindlimb immobilization of animals with mild to moderate spinal cord injuries sufficiently disrupts the patterns of afferent input from the hindlimbs to prevent the maintenance of the acute capacity leading to a dramatic drop in locomotor function over the first few weeks post-injury. With the caveats that intense acute 
activity may be detrimental to traumatically injured spinal cord (Smith et al., 2009), our results support suggestions that appropriate activity, applied acutely, may enhance or optimize overall functional outcomes following a contusive spinal cord injury.

\section{Conclusion}

The current findings suggest that limiting early in-cage activity can dramatically alter functional recovery of locomotion in a rodent model of mildmoderate spinal cord injury. Surprisingly, we also show that a hindlimb stretch protocol can have a negative effect on recovery in normally housed animals, preventing the development of forelimb-hindlimb coordination, while having no apparent influence on recovery in hindlimb immobilized animals or on the development of ankle contractures. These findings imply that the immobility experienced by the majority of spinal cord injured patients, and some aspects of standard of care therapies, may significantly hinder functional recovery acutely, and also potentially limit the responsiveness of the spinal cord circuitry to training efforts initiated at later time points. 


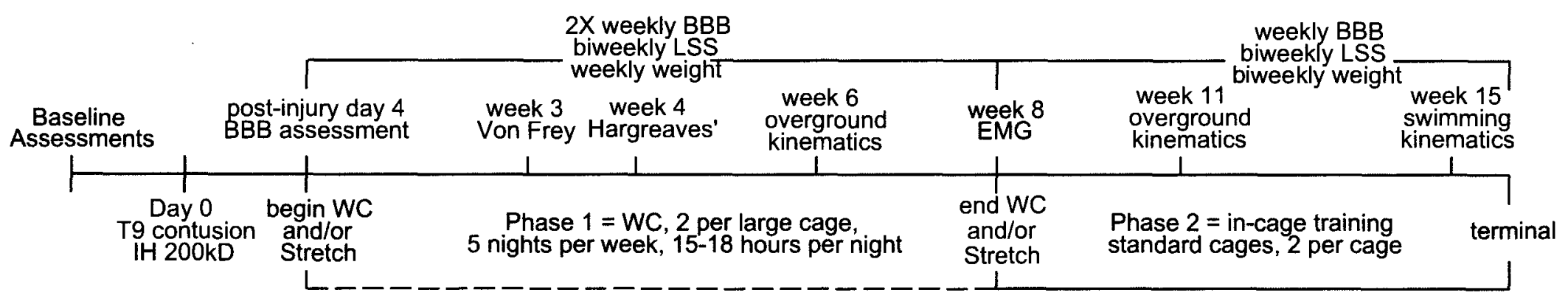

Figure 1. Timeline WC Hindlimb Immobilization.

Wheelchair immobilization and stretch protocol was for an 8 week period (dashed line) followed by 8 weeks of normal/standard ( 2 per cage) housing. The schedule of injury and treatments are shown below the timeline and the schedule of assessments is shown above. 
Figure 2. BBB Scores WC Hindlimb Immobilization.

A. Shown is an injured animal in a wheelchair. Velcro straps held the animal and hindlimbs in place. B. Shown is a 3D kinematic analysis of the right hindlimb during wheelchair immobilization. The anatomical landmarks represented are the iliac crest (IC), hip, knee, ankle and toe. Some movement of the knee or ankle was sometimes seen during spasms, as shown here for the knee. C. Shown are the BBB scores over time for all 4 experimental groups (wheelchair - WC; stretch $\mathrm{SR})$. All animals scored 21 pre-injury. Significant differences were found for both WC groups ( $n=5$ each) compared to non-WC/non-SR controls $\left({ }^{*}, n=6, \pm S D, p \leq .05\right)$. Animals receiving the $S R$ intervention (non-WC/SR, $n=5$ ) had BBB scores that were significantly different from non$W C / n o n-S R$ controls $(* *, n=6, \pm S D, p \leq .05)$. D. Shown are the BBB scores over time for groups based on presence (con) or absence (no-con) of ankle contractures. WC/con $(n=3)$ and WC/nocon $(n=4)$ groups have significantly lower BBB scores compared to controls for weeks 2-16 ( ${ }^{*}$, $n=6, \pm S D, p \leq .05)$. Scores for the WC/con group are significantly different from the WC/no-con group at weeks 2,6 and $10-16(\&, n=3 / 4, \pm S D, p \leq .05)$.
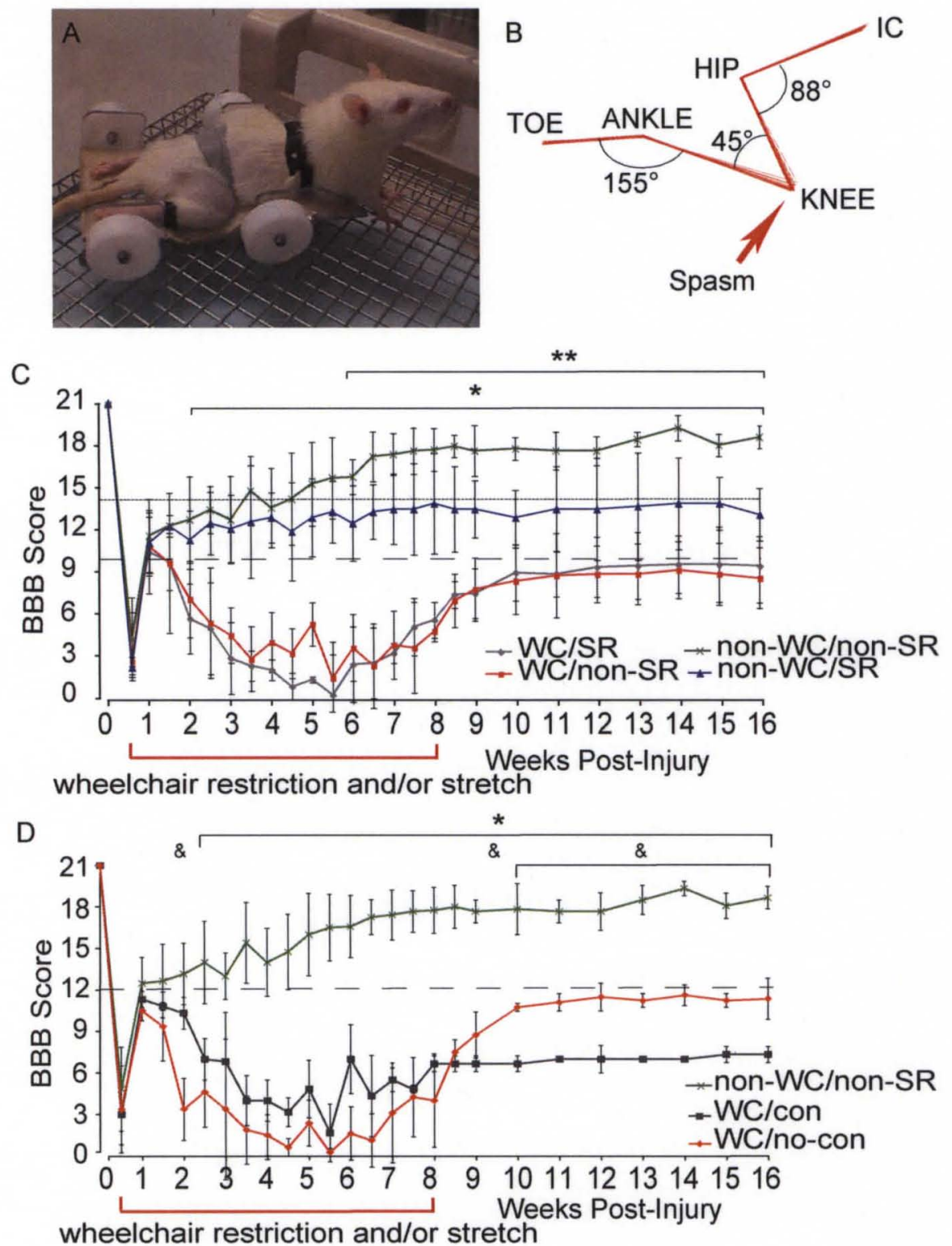


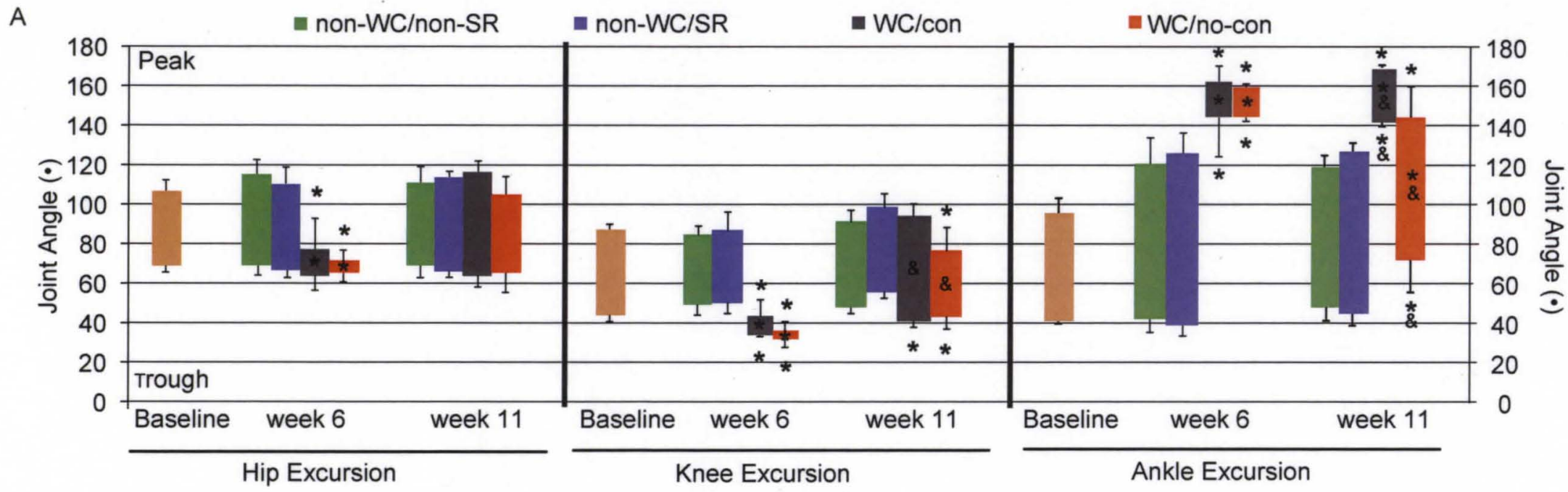

Figure 3. Intralimb Coordination Overground Stepping WC Hindlimb Immobilization.

A. 3D kinematic analysis of hindlimb joint excursions overground stepping. Joint excursion is presented as the range of angles through which the joint moves and is calculated as maximum peak angle (extension) minus the trough angle (flexion). Baseline excursion data represents the pre-injury average for all animals. Symbols within each bar represent significant differences in average excursion, symbols above and below represent significant differences in average peak or trough angles; $\left(^{*}\right)$ indicates significant differences for both WC groups (WC/con, $n=3$ and $W C / n o-c o n, n=4$ ) as compared to both non-WC groups (non-WC/SR, $n=5$ and non-WC/non-SR controls, $n=6, \pm S D, p \leq .05)$, and $(\&)$ represents significant differences between the two WC groups $(n=5 / 5, \pm S D, p \leq .05)$. 

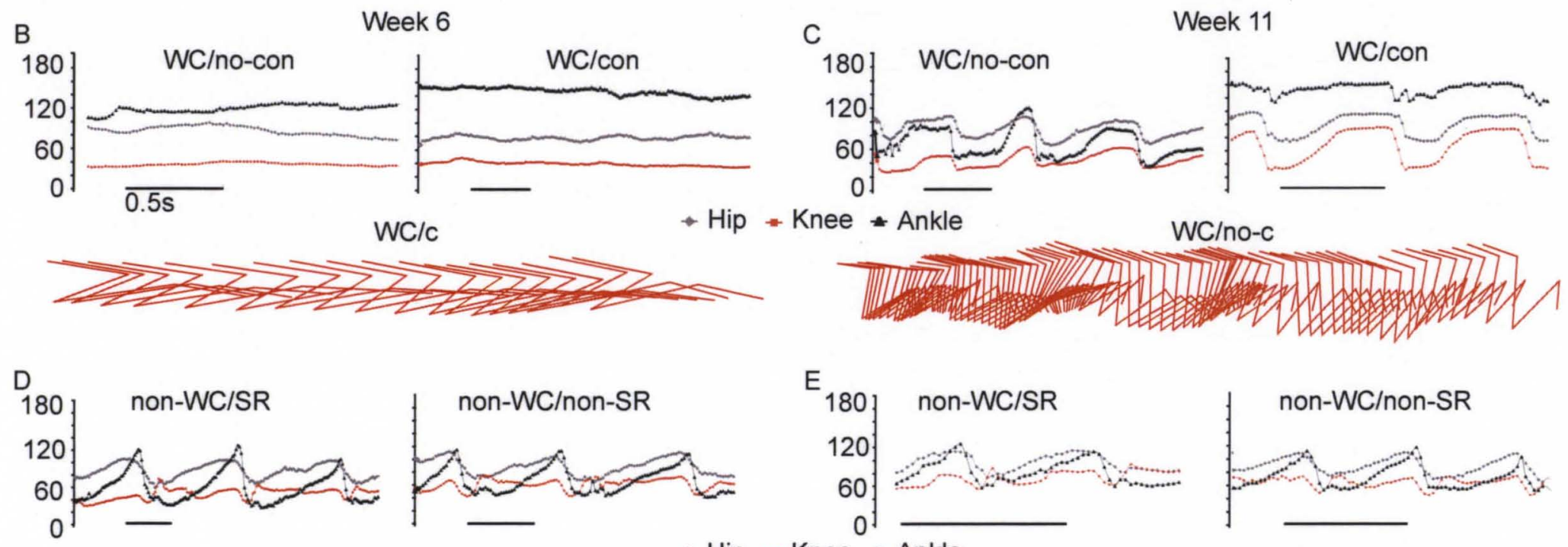

E
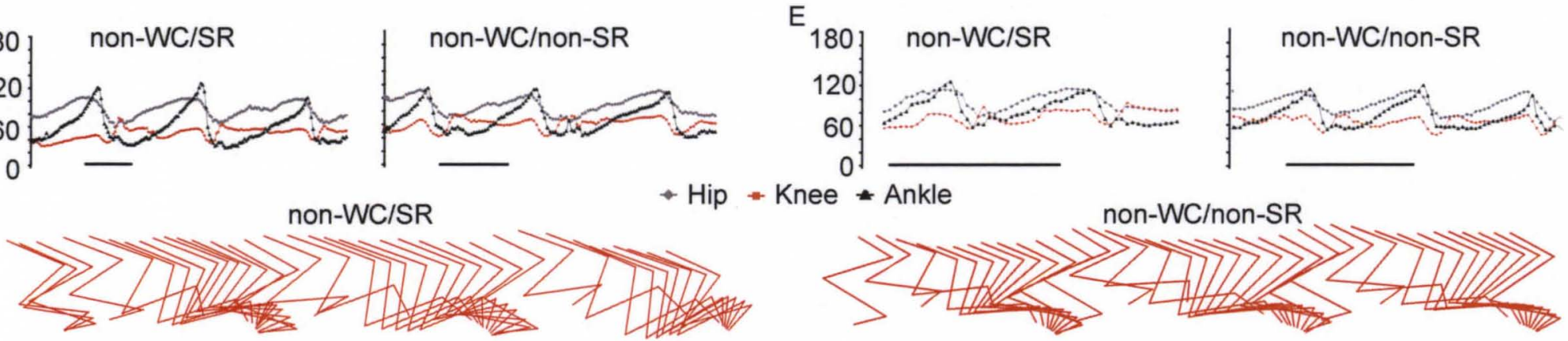

Continued Intralimb Coordination Overground. B through E shows two examples of excursion plots (joint angle changes over time) and examples of stick figures. B and $\mathrm{C}$ show excursion plots for WC rats with and without contractures and stick figures showing extended-limb dragging (B) and flexed limb dragging (C) in WC animals with and without contractures, respectively. WC/con animals displayed cyclic hip and knee movement despite the reduced ankle ROM. D and E show excursion plots and stick figures for nonWC animals that represent near normal stepping of the hindlimbs that was not different from baseline at either time point. 
Figure 4. Swimming Assessments WC Hindlimb Immobilization.

A. Both WC groups, regardless of ankle contracture, had significantly lower LSS scores compared to non-WC/non-SR controls for weeks $3,5,7$ and $11\left({ }^{*}, n=3,4 / 6, \pm S D, p \leq .05\right)$. Following the 8 weeks of WC and/or SR interventions (dashed line under x-axis), WC/no-con animals experienced a sharp gain of swimming function (WC/no-con, weeks 9 and 15, peak LSS score of $9.50 \pm .903$ ), and only WC/con animals had significantly lower LSS scores (LSS 6) compared to controls $\left({ }^{*}, n=3 / 6, \pm S D, p \leq .05\right)$. B. The LSS subscore indicates that both WC groups lacked the ability to produce hindlimb locomotor strokes as compared to controls (weeks 3, 5 and 7 during WC and/or SR interventions, and weeks 11 and 13 after the interventions had ended $\left({ }^{*}, n=3,4 / 6, \pm S D, p \leq .05\right)$. WC/no-con animals showed significant improvement in hindlimb function during swimming once WC and SR interventions ceased, weeks 9 and 15 ( $p \leq .05)$.

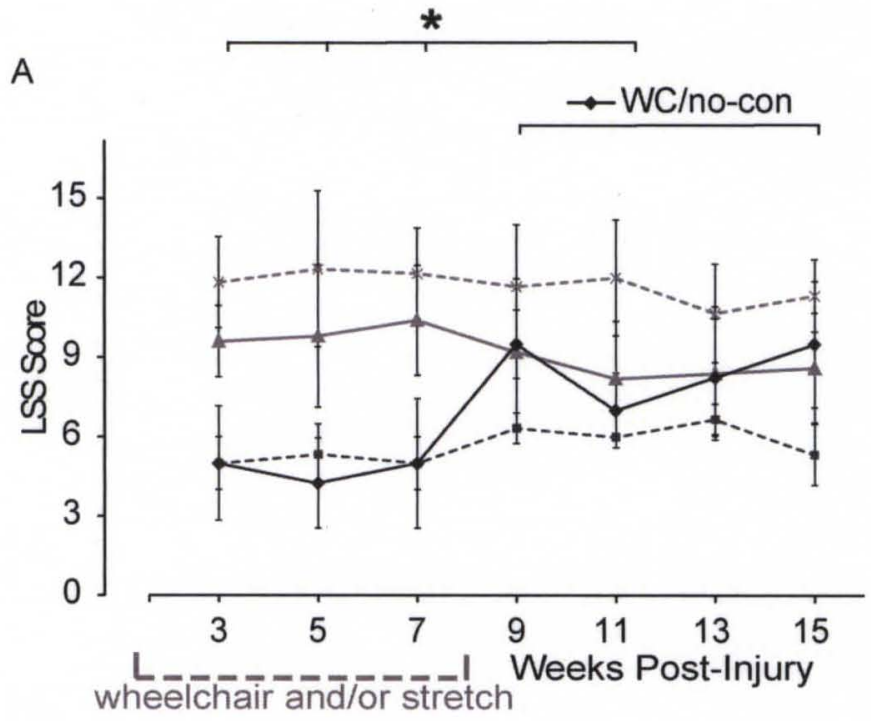

B

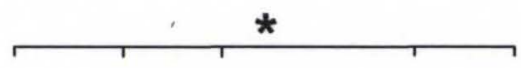

$$
\begin{aligned}
& -- \text { WC/con } \\
& \rightarrow \text { WC/no-con } \\
& \star \text { non-WC/SR } \\
& --* \text { non-WC/non-SR }
\end{aligned}
$$
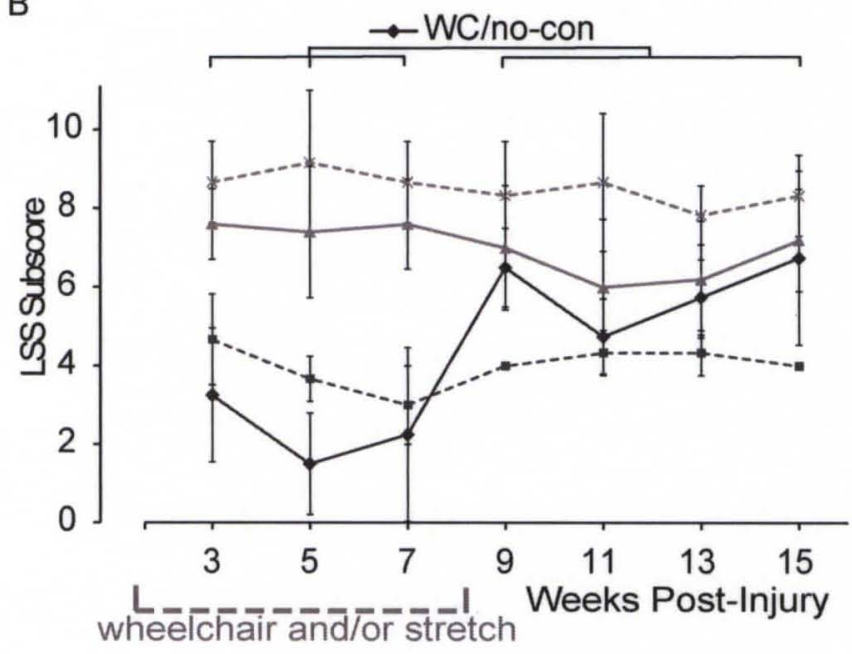

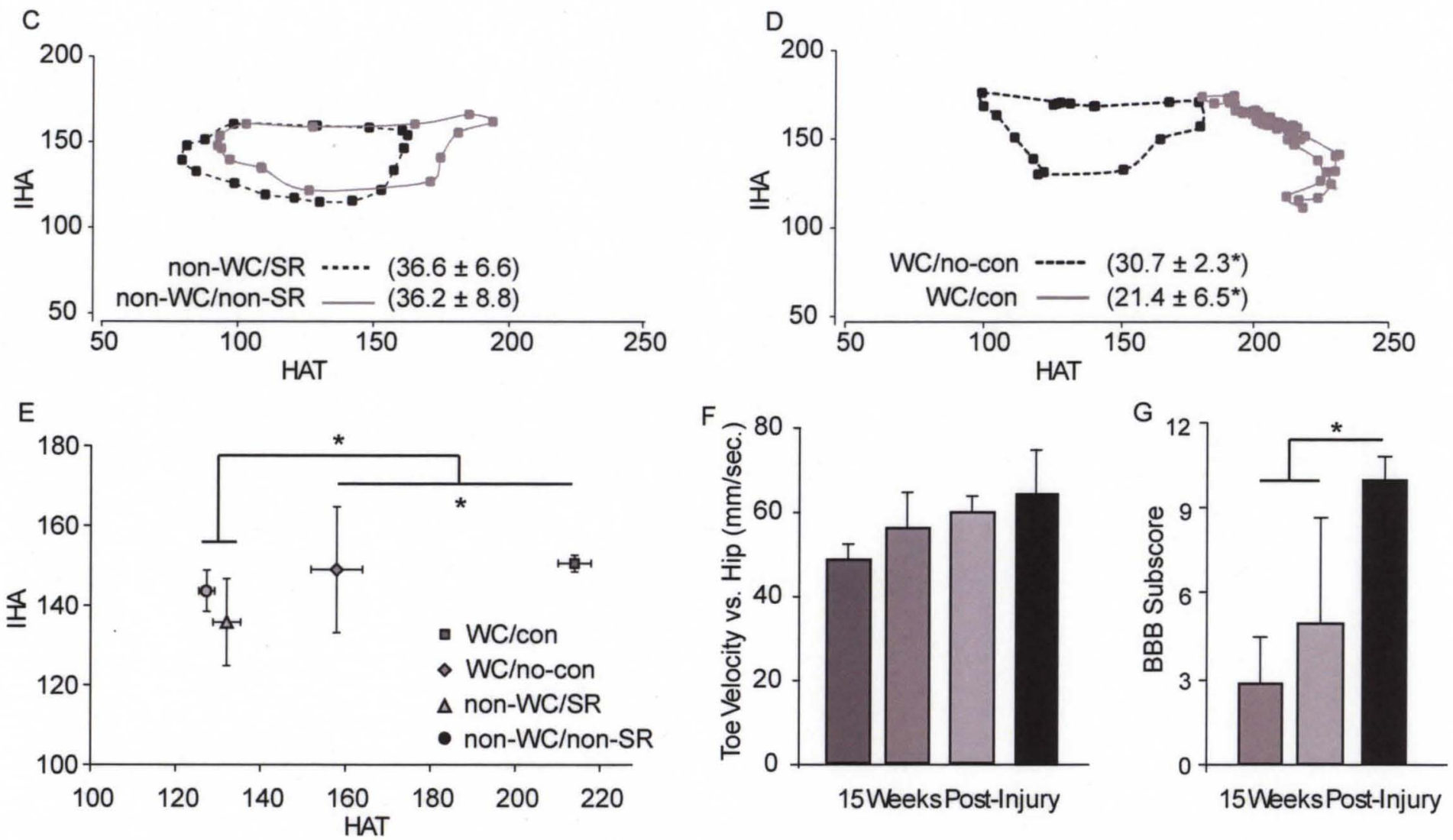

Continued Swimming Kinematic Assessment. C and D. Shown are sample (single strokes from one animal) angle-angle plots (IHA vs. HAT) for non-WC (C) and WC (D) groups and the average plot areas as calculated using elliptical Fourier analysis, which were significantly different based on the presence/absence of contractures (WC/con, $n=3$ and WC/no-con, n=4, ${ }^{*}, \pm S D p \leq .05$ ). E. The centroids of the angleangle plots showed significant differences for WC/con and WC/no-con HAT angle, compared to both non-WC groups at 15 weeks postinjury $\left({ }^{*}, \pm S D, n=3,4 / 5,6, p \leq .05\right)$. F. Kinematic analysis of the peak velocity of the toe, relative to the hip, shows that all four groups (based on contractures) could generate velocities greater than $40 \mathrm{~mm} / \mathrm{sec}$, and there were no significant group differences. G. As a complement to the LSS subscores (hindlimb components only), we determined the subscores for the BBB Scale (at 15 weeks post-injury) and found that both the WC and non-WC/SR groups retained significant deficits in the fine aspects of stepping. 
Figure 5. In-vivo Micro-CT Bone Architecture WC Hindlimb Immobilization.

A. In-vivo Micro CT-scans of cortical and cancellous bone of the proximal tibial metaphysis showed no significant differences between WC immobilized animals and controls. B. \& C. Sample scans of the proximal tibial metaphysis and lateral condyle from one non-WC $(B)$ and one $\mathrm{WC} /$ no-con $(\mathrm{C})$ animal. Arrows indicate apparent differences in the trabecular density in the shaft and cortical bone of the lateral condyle.

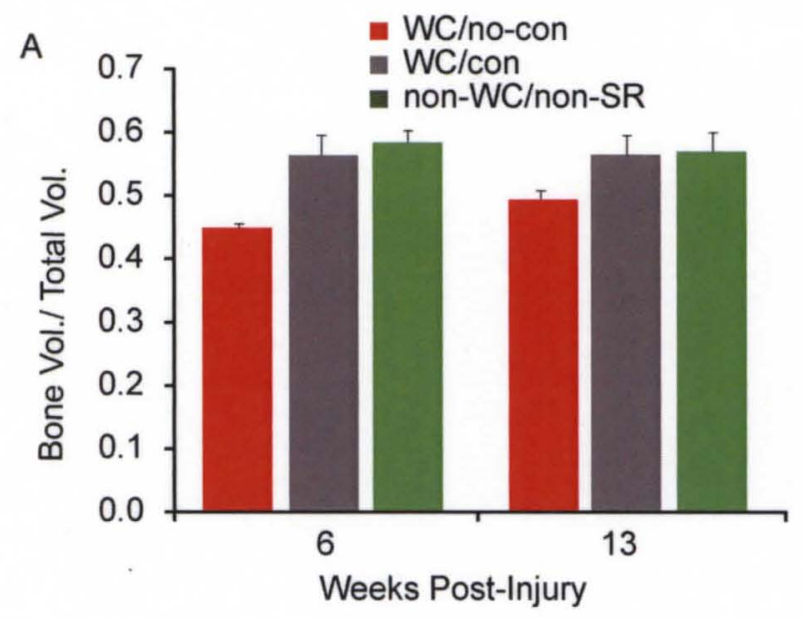

Images of MicroCT Scans of Proximal Tibia non-WC/non-SR

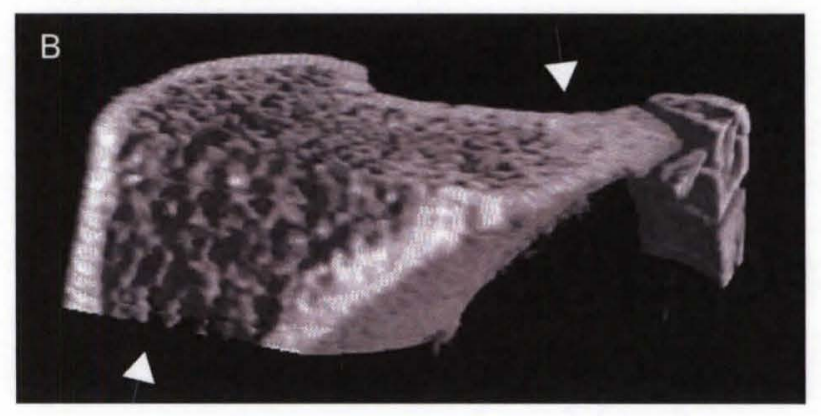

\section{WC/no-con}

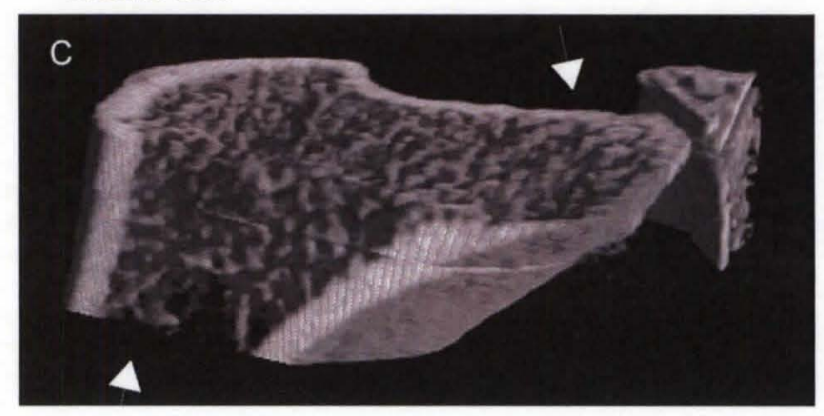


Figure 6. Muscle Weight WC Hindlimb Immobilization.

A. Wet weights of the left tibialis anterior (TA muscles) were not different for the WC and nonWC groups, however, the left gastrocnemius muscles (medial and lateral dissected together) from the WC groups were significantly lighter than those of the non-WC groups ${ }^{*}, n=5,5 / 5,6$, $\pm \mathrm{SD}, \mathrm{p} \leq .05)$. B. Sciatic nerve cross-sectional area was significantly lower for WC animals that did not receive stretch as compared to non-WC animals that did receive stretch (WC/non-SR, $0.104 \pm 0.0136 n=5$ vs. non-WC/Stretch $\left.0.148 \pm 0.0146,{ }^{*}, n=5 / 5, \pm S D, p \leq .05\right)$.
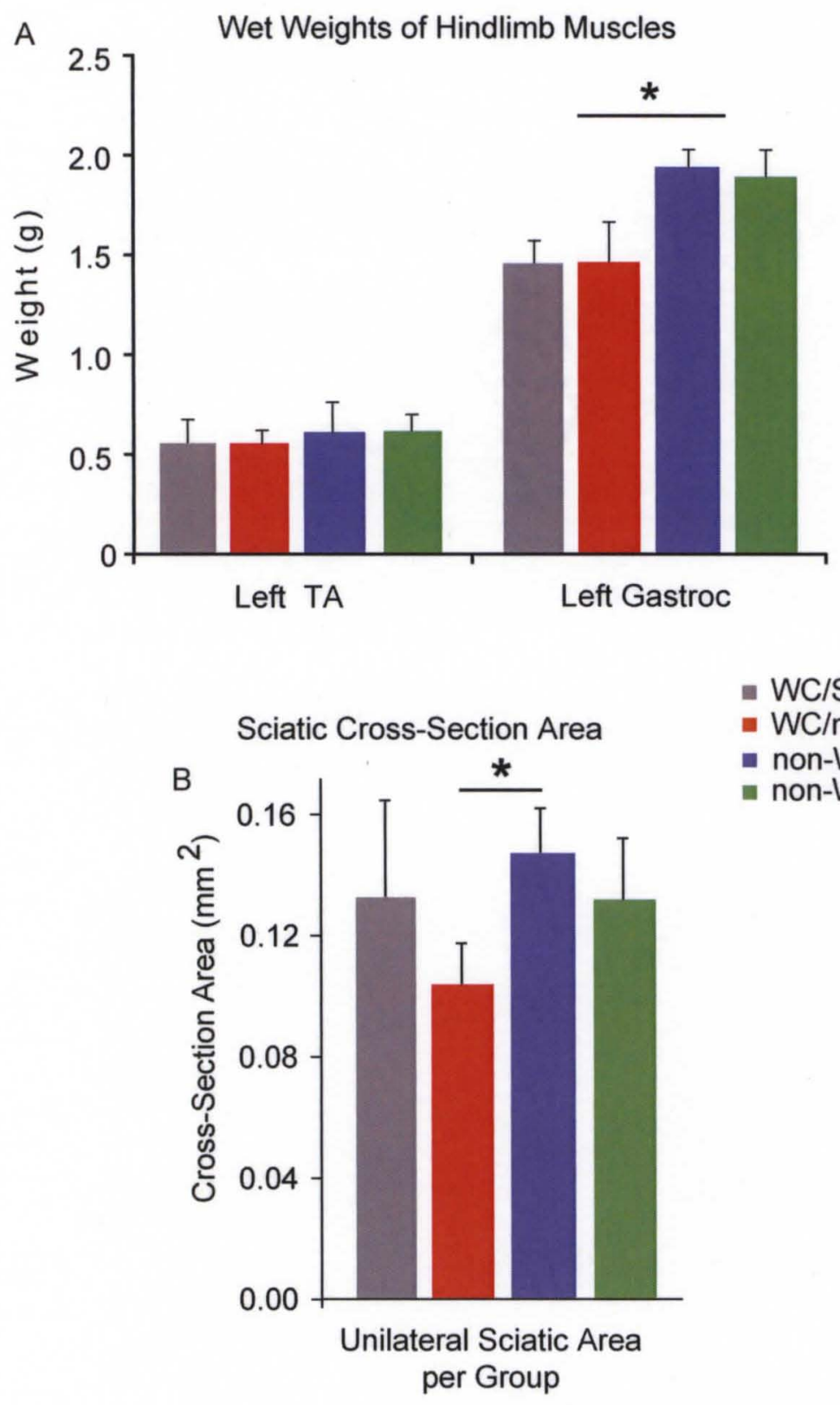

- WC/SR

- WC/non-SR

- non-WC/SR

- non-WC/non-SR 
Figure 7. Muscle Co-contraction WC Hindlimb Immobilization.

The percent co-contraction of right and left LG muscles was calculated from EMG recordings from each group for both tail and paw pinch conditions. WC animals had significantly greater co-contraction during tail pinches as compared to non-WC (SR and non-SR, ${ }^{*}, n=10 / 11$

respectively, $\pm S D, p \leq .05$ ).

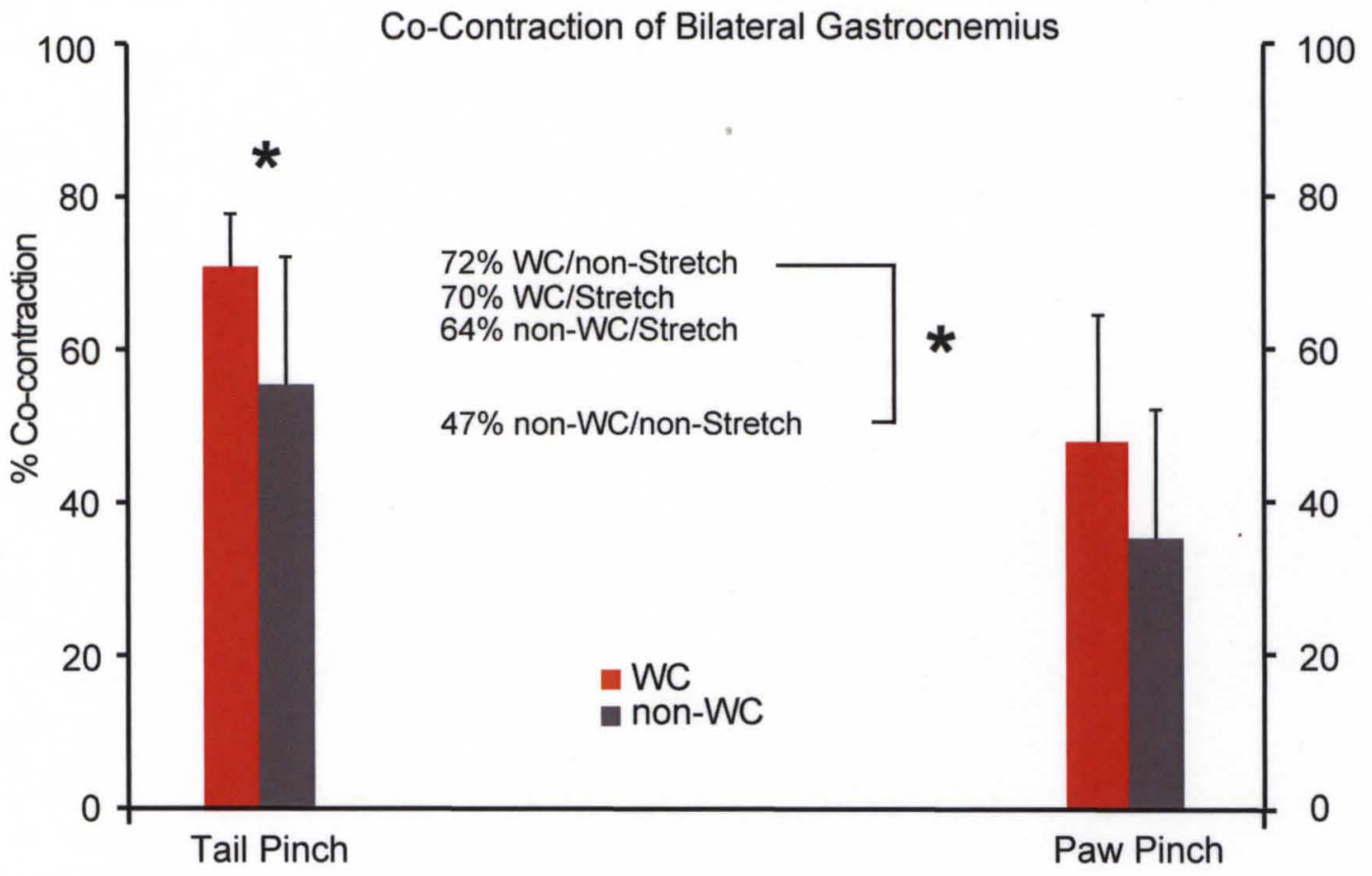


Histological Analysis of Injury Epicenter.

\begin{tabular}{llcl}
\hline Group & White Matter CSA & Gray Matter Volume & Cavity Volume \\
\hline WC/SR & $28.8 \pm 14.13$ & $160.2 \pm 24.75$ & $17.2 \pm 7.72$ \\
\hline WC/non-SR & $21.4 \pm 7.16$ & $152.3 \pm 30.03$ & $21.6 \pm 21.08$ \\
\hline non-WC/SR & $24.4 \pm 7.10$ & $152.7 \pm 52.62$ & $36.8 \pm 27.38$ \\
\hline non-WC/non-SR & $32.4 \pm 6.36$ & $143.8 \pm 25.85$ & $53.7 \pm 29.8$ \\
\hline
\end{tabular}

Table 1. Histology WC Hindlimb Immobilization.

Shown is the mean spared white matter cross-sectional area (CSA) at the injury epicenter $\left(\mathrm{mm}^{2}\right)$, gray matter volume loss (mm ${ }^{3}$ ) and cavity volume $\left(\mathrm{mm}^{3}\right)$. No significant differences were found among groups. 


\section{CHAPTER III}

\section{FUNCTIONAL LOCOMOTOR RECOVERY IS NEGATIVELY ALTERED IN A TASK-SPECIFIC MANNER FOLLOWING CONTUSIVE SPINAL CORD INJURY IN THE ADULT RAT}

\section{INTRODUCTION}

The concept of task-specificity in activity-based rehabilitation posits that general activity does not promote learning of skilled actions, but it is both the quality and intensity of specific sensorimotor afferent information that engages neural circuits and induces skilled motor learning (Basso and Hansen, 2011). Body weight supported treadmill (BWST) step training studies in completely transected animals have repeatedly demonstrated that activity-based rehabilitation leads to improved abilities exclusive to the trained task. Separate components, or qualitative aspects, of afferent input such as proprioception and pattern of cyclic hindlimb movements, cutaneous paw input, and extensor muscle loading determine different features of functional locomotor recovery. For example, spinalized cats can be trained to stand, but their ability to step on a treadmill deteriorated; conversely, if stepping was trained the positive effects of stand training were not evident (Edgerton et al., 1992; Hodgson et al., 1994; de Leon et al., 1998; de Leon et al., 1999b; Tillakaratne et al., 2002). Combining the afferent components of increased extensor loading with specifically patterned phasic inputs during step training resulted in greater locomotor recovery on a 
treadmill in the complete transected cat model (Timoszyk et al., 2005), as well as in patients stepping on a treadmill (Harkema et al., 1997; Kojima et al., 1999; Harkema, 2001, 2008; Harkema et al., 2012b). Oppositely, swim training provides a high number of locomotor cycles (high intensity) and can be combined with cutaneous input, but does not require extensor loading (a quality or component of afferent input). The ability to weight support during overground stepping was not improved with swim training, but after only a few weeks of daily swim training the pattern of intralimb coordination during in an incomplete lower thoracic contusion injury (Smith et al., 2006a; Smith et al., 2006b; Magnuson et al., 2009). Proper intensity refers to optimizing the amount (or duration of) and thus the repetition of specific afferent input during a training regimen. A certain minimum amount of step training with specific sensorimotor input is essential to bring about the desired improvements in hindlimb trajectory, speed of stepping, and coordination (Edgerton et al., 2004; Majczynski and Slawinska, 2007). Studies from the de Leon lab have shown in a neonatal rat transection model that treadmill step training sessions with 1000 , versus only 100 , steps resulted in higher quality locomotor recovery, increased ability to perform more weightsupported steps at faster treadmill speeds, and improved hindlimb trajectory (Cha et al., 2007; de Leon et al., 2011). Unfortunately, in all these studies the positive effects of activity-based training does not translate to increased ability to perform voluntary unassisted stepping. As Marsh and colleagues have outlined, sufficient retention of the task is evident since trained animals can repeatedly perform stepping, but generalization of the specific cues and conditions of 
practice on treadmill may represent the greater hurdle to transferring the skilled locomotor performance to overground stepping (2011).

Treadmill step training is task-specific in that it provides a moving platform that induces extension of the hip to cue the CPG to produce locomotor output (Dobkin et al., 1995), while overground stepping takes cues from balance and vestibular systems as well as joint extension (Dobkin and Duncan, 2012). The relative benefits of overground step training versus higher intensity BWST step training are debatable (Hornby et al., 2005; Wirz et al., 2005; Wessels et al., 2010), but regardless of the methods used, increasing volitional control of movement is the primary goal. Our laboratory recently studied shallow water walking (SWW) as a model of activity-based rehabilitation after T9 contusion injuries in the rat, and was developed with respect to the history and summation of years of treadmill step training data from transected cat and rat studies. SWW represents a seemingly optimal combination of voluntary quadrupedal stepping: partial weight support and lateral stabilization provided via buoyancy in the water, the water level is adjustable to challenge the extensor loading and weight support during training, and cutaneous feedback provided from the textured surface of the training tank (Kuerzi et al., 2010). There were significant task-specific improvements for animals that were SWW trained in that hindlimb kinematic trajectory approached normal and increased plantar weight-supported stepping, but only while stepping in the shallow water, and no improvement in overground stepping ability was evident (Kuerzi et al., 2010). Results from SWW training parallel treadmill step training studies showing robust improvements due to 
training but only when stepping on a treadmill. These studies indicate that spared spinal cord circuitry is capable of producing motor output when given a specific environment with all the proper cues provided extraneously. However, to be clinically relevant an activity-based training paradigm should result in training of volitional control of a skill that can also be applied to other tasks and environments (Basso and Hansen, 2011). We have not yet elucidated the best combination of afferent input qualities and intensity of training to produce an activity-based locomotor rehabilitation strategy consistent and robust enough for $\mathrm{SCl}$ patients whose goal is to walk independently again.

The expected results from transection studies have been elusive in $\mathrm{SCl}$ patients. An obvious difference is that in both transection and incomplete $\mathrm{SCl}$ animal models training is initiated acutely, within the first week, a clear distinction from $\mathrm{SCl}$ studies on patients in which the earliest activity-based rehabilitation began was 4.5 weeks post-injury on average (Dobkin et al., 2006; Dobkin et al., 2007). The impact of this may be most evident when considering the natural course of recovery of rats with incomplete $\mathrm{SCl}$. As introduced in Chapter 2 (Caudle et al., 2011), functional locomotor recovery in these animals has been recognized, by our lab and others, as early spontaneous recovery that is not receptive to activity-based locomotor training aimed to improve overground stepping (Fouad et al., 2000; Smith et al., 2006a; Heng and de Leon, 2008; Magnuson et al., 2009; Kuerzi et al., 2010; Alluin et al., 2011). There are no additional benefits of an activity-based rehabilitation strategy because of a ceiling effect; the locomotor recovery before the training is applied is already substantial 
(Kuerzi et al., 2010). We developed a model of wheelchair immobilization in an effort to test the hypothesis that rats with low thoracic contusive $\mathrm{SCl}$ achieve maximal locomotor recovery due to auto-training (or in-cage training). In our previous study we found that rats intermittently wheelchair immobilized, overnights only for 5 nights a week for 8 consecutive weeks, gained substantial locomotor recovery (BBB 12) by 1 week, but subsequently experienced a marked loss of locomotor recovery that persisted at least to sixteen weeks (Figure 2). If auto-training within the first few weeks post-injury contributes to early locomotor recovery, then preventing auto-training 24 hours per day $/ 7$ days per week (24/7) may prevent the initial gain of locomotor function. To test this notion, moderate contusions $(12.5 \mathrm{~g}-\mathrm{cm})$ using the NYU impactor were performed at the T9 segmental level, and experimental groups consisted of a non-WC group allowed to recover in standard cages for 8 weeks, and a 24/7 wheelchair group starting at 4 days post-injury through 6 weeks. In an effort to prevent the pathological ankle contracture produced in the previous WC study, wheelchairs used in the current study were designed to allow the hindlimb joints freedom to move within a limited range-of-motion but still prevented extensor loading and plantar paw contact in order to test task-specificity of recovery. WC animals were delayed in locomotor recovery of plantar stepping as measured by the BBB Open-Field Locomotor Scale and 3D kinematic analysis of overground and shallow water walking. Our data suggests that functional locomotor recovery is limited in a task-specific manner, and even minimal amounts of proprioceptive afferent input promotes locomotor recovery specific to intralimb coordination. 


\section{MATERIALS AND METHODS}

Spinal Cord Injury and Experimental Design

Ten female adult Sprague-Dawley rats (190-215g, Harlan) were used for

this study. All procedures involving experimental animals were performed according to the guidelines of the University of Louisville Institutional Animal Care and Use Committee. Prior to injury, animals were randomly assigned to the experimental group: ( $24 / 7$ in wheelchairs $(W C), n=5)$ or to the control group (non $/ \mathrm{WC}, \mathrm{n}=5$ ). Each animal was anesthetized with pentobarbital $(55 \mathrm{mg} / \mathrm{kg}$ i.p.) and given prophylactic antibiotics (Gentamicin sulfate $15 \mathrm{mg} / \mathrm{kg} \mathrm{sc}$.). A single level laminectomy was performed at the T9 vertebrae and a moderate contusion injury $(12.5 \mathrm{~g}-\mathrm{cm})$ was performed at the T10 cord level using the NYU Impactor (W. Young, Rutgers University, NJ) as previously described (Magnuson et al., 1999). Body temperatures were maintained at $36-37^{\circ} \mathrm{C}$ throughout the procedure and until the animals had recovered from anesthetic. After injury, wounds were closed in layers using silk sutures with topical antibiotics applied to the incision. Animals remained in recovery cages on heating pads until fully recovered from anesthesia. Upon waking from contusion surgery, day 0 , non-WC animals were housed socially, two or three per cage, in standard cages. All cages included Alpha Dry bedding with food and water provided ad libitum, all animals were housed in the same room with 12-hour light dark cycle and received daily postoperative care, including manual bladder expression as needed. Wheelchair use began at 4 days post-injury, and continued for 24 hours per day $/ 7$ days per week, 
for duration of 6 weeks. WC cage mates stayed together, paired, in large breeding cages while non-WC animals remained paired in standard cages. All assessments continued until 8 weeks post-injury, at which point all animals were sacrificed (terminal).

Wheelchairs used in this study were designed to hold the trunk in a stationary position with free movement of the forelimbs to allow for maneuvering about the cage. Wheelchairs were constructed as previously described (Chapter 2 methods) however in the current study a lightweight thermoplastic material was used for the platform. The thermoplastic was shaped into a series of bends along the caudal half of the wheelchair allowed the hindlimb joints to move through a range-of-motion without achieving weight support or paw stimulation (Figure 8A). A representative sample of unilateral kinematic analysis (see methods below) of a hindlimb at rest (bold red lines) while in a wheelchair is shown in Figure 8B. The rays originating from each joint (shorter red lines) represent the range of possible positions each limb segment could achieve. Velcro straps around the ribcage, hips and both ankles kept the animal securely fastened. Wheelchairs were cleaned daily and no animal exhibited autophagia.

\section{Daily Care}

All animals received standard post-operative and daily care as described in Chapter 2 methods. Briefly, WC animals were taken out of wheelchairs daily and immediately received a daily 2-minute massage of the hindlimbs in a distal to proximal direction for pressure relief and to aid in circulation (non-WC controls also received the daily 2-minute massage). Skin care was proactive and straps 
were adjusted several times per 24-hour period for inspection; topical antibiotics and layers of fresh clean gauze were applied frequently. The criterion for temporary exclusion from the wheelchair beyond 2 hours was a skin abrasion with non-blanchable redness, with or without sloughing of the epidermis, which did not respond to topical antibiotics within 8 hours. Only 2 out of 5 WC animals were temporarily excluded on 2 occasions each for no more than 48 hours per occasion. Immediately following daily care animals were allowed to recover in a standard cage with bedding for no more than two consecutive hours. On occasion, WC animals were in standard cages during or in between behavioral testing sessions. We closely monitored general health and provided NutriCal supplement to WC animals frequently. Animals were weighed preoperatively and every 4 weeks thereafter. Hindlimb muscles tibialis anterior (TA, ankle flexors), lateral/medial gastrocnemius (LG/MG, ankle extensors), biceps femoris (BP, knee flexors), and vastus lateralis/medialis/intermedius, and rectus femoris (quadriceps, knee extensors) were harvested upon termination.

\section{Behavioral Assessment}

Hindlimb movement during overground stepping was assessed using the BBB Open Field Locomotor Scale as previously described (Kuerzi et al., 2010). The low end of the scale is characterized by movements of individual hindlimb joints without weight-supported stepping, and the high end of the scale is characterized by consistent weight-supported stepping, consistent coordination, toe clearance, paw position and tail position (Basso et al., 1995). BBB evaluation was performed preoperatively, on day 4 , week 1 and every other week thereafter 
to minimize any possible effect of auto-training during stepping assessment. The animals were presented at random to the same two observers, both blinded to experimental groups, for all behavioral scoring.

The Louisville Swim Scale (LSS) is a $0-17$ point scale designed in our laboratory to assess swimming performance during 4 minutes of lap swimming as previously described (Smith et al., 2006b). Swimming assessments were performed preoperatively, once at post-injury week 1 and then terminally at 8 weeks. The animals were briefly reintroduced to the water two days prior to every LSS assessment to decrease stress response. Briefly, LSS scores in the 0-5 range indicates poor swimming with little or no hindlimb movement, dependence on their forelimbs for forward motion, and severe trunk instability (body rotation) and/or body angle (the animals back is far below the water surface). Intermediate scores (6-11) indicate retained dependency on forelimbs for forward movement with varying degrees of hindlimb movement, trunk instability, and body angle. Animals that score greater than 12 have consistent hindlimb movement, little to no forelimb dependency, frequent to consistent alternating hindlimb movements, less trunk instability and body angle. The LSS subscore has a maximum score of 11 points and accounts only for the hindlimb cycling abilities by excluding the trunk stability and body angle portions of the assessment.

ROM of the Ankle

The movement trajectory (quality) and gait (pattern) of locomotor output results from the status of the central and peripheral nervous system as well as from peripheral musculoskeletal structures. Joint capsule, ligament, soft tissue 
and muscle tightness, joint extensibility, as well as joint or bone abnormalities are all possible contributors to limited ROM (O'Sullivan and Schmitz, 2001). Passive range-of-motion (pROM) is motion across a joint that is performed by an examiner without the assistance of the animal/subject. Since we saw severe ankle contracture in WC animals in the previous study we focused on measuring pROM flexion and extension of the ankle, using a universal goniometer (Figure $8 C)$, on day 4 and every two weeks thereafter. Each rat was wrapped in a terry cloth towel such that only the hindlimbs were accessible for passive manipulation. The knee was bent at $\sim 75^{\circ}$ flexion while 2 examiners measured ankle pROM using the goniometer aligned on toe, ankle and knee bony landmarks. In the case of discrepancy both examiners had to come to an agreement on the final measurements and rounded to increments of $5^{\circ}$. Total pROM for the ankle is $135^{\circ}, 15^{\circ}$ of flexion and $150^{\circ}$ of extension.

Kinematic Assessment of Stepping, Swimming and Shallow Water Walking To quantitatively assess intralimb coordination during overground locomotion, three-dimensional (3D) kinematic analysis was performed at 2, 4, 6 and 8 weeks. Bilateral hindlimb trajectory was averaged for both sides from a minimum of 3 passes on each side in which the animal did not hesitate, or at lease 5 full step cycles. Data are presented as averaged total angle excursions with the peaks of the angle corresponding to the greatest amount of extension and the troughs as the greatest points of flexion through the step cycles. Bony landmarks of hindlimb iliac crest $(I)$, hip $(H)$, ankle $(A)$ and toe $(T)$ were marked, as previously described (Magnuson et al., 2009; Kuerzi et al., 2010), and 
hindlimb movement was recorded with two Basler $602 \mathrm{f}$ high-resolution digital cameras running at $60 \mathrm{~Hz}$ connected to a $\mathrm{PC}$ using the video software DVR Explorer (Advanced Digital Vision, Natick, MA). The digital AVI files from both cameras were opened in MaxTraq3D (Innovision Systems, Columbiaville, MI) where they were digitally identified in a semi-automated fashion on each frame. The two dimensional files were digitally combined to form a three dimensional file (.mqf) from which all stick figures and angle excursion data is derived in Excel or Maxmate (Innovision Systems). At week 4 we used 3D kinematic analysis to capture movement of the hindlimbs while the rats were in their wheelchairs. Stick figure representations were derived using the Maxmate plug in for Excel as described above.

Kinematic assessment of intralimb coordination during shallow water walking (SWW) was performed at 2, 4, 6 and 8 weeks, but swimming was assessed only at baseline and week 8 . As previously described, the bony segments are marked and digitally identified using side-view 2D kinematic analysis (Chapter 2). Three hindlimb segments, iliac crest (IC) to hip $(H)$, hip $(H)$ to ankle (A), and ankle (A) to toe (T), represent the phase relationships of hindlimb trajectory during SWW and swimming cycles in the form of peak, trough and excursion data described above. The relationship of the two angles formed by the segments (IHA, HAT) form an ellipsoid that is a visual representation of the hindlimb through a swimming stroke cycle (Magnuson et al., 2009). Averaged swim stroke cycles per group are represented as centroid plots of the relationship of IHA and HAT angles. 
Interlimb coordination was assessed during SWW from ventral view digital recordings using a single camera (2D gait analysis) mounted under the Plexiglas clear tank filled with $5 \mathrm{~cm}$ water. As described for the traditional footprint analysis technique (Kunkel-Bagden et al., 1993) the plantar surface of each paw was digitally marked using the same video software described above. Digital identification of plantar and dorsal steps, paw placement order and coupling was calculated as previously described (Kuerzi et al., 2010). Regularity Index (RI) originally described by Hamers and Koopmans (2001; Koopmans et al., 2005) was calculated as the number of correctly patterned plantar step cycles/total cycles (dorsal and plantar cycles) and gives an indication of interlimb coordination of plantar only stepping. For rodents with $\mathrm{SCl}$ that have no reasonable prospect for coordinated stepping we adapted the Plantar Stepping Index (PSI) (Cheng et al., 1997; Kuerzi et al., 2010). PSI is calculated as the number of hindlimb plantar steps/forelimb plantar steps and indicates how consistently the animal is able to achieve plantar stepping. Since animals in our model are capable of producing consistent dorsal stepping we have developed the Central Pattern Index $(\mathrm{CPI})$ to indicate the quality of coordination during dorsal stepping. CPI is calculated as the number of correctly patterned cycles (dorsal and plantar cycles) divided by the total number of cycles (dorsal and plantar cycles) with a "rolling" reference paw. This value indicates whether the animal can achieve coordination regardless of the ability to properly place the paw on its plantar surface. Using a rolling reference paw makes the calculation more sensitive to incorrect patterns and double steps with the same paw. 
Interlimb coordination was also assessed using the timing of the stance phases (homolateral, homologous, and diagonal) relative to an entire step cycle (Leblond et al., 2003). The coupling relationship represents the percentage of the step cycle that any two limbs are in stance; presumably weight-supporting plantar or dorsal stepping based on $12.5 \mathrm{~g}-\mathrm{cm}$ T9 injuries in the current study. Couplings were calculated for uninjured rats: homolateral coupling (on the same side of the animal, $\sim 50 \%$ ) homologous coupling (limbs on the same girdle, $\sim 60 \%$ ) and diagonal coupling (LF vs. RH and RF vs. $\mathrm{LH}, \sim 90 \%$ ). Additionally, we calculated a diagonal distance measurement to assess the how stride length might be affected by compensatory intralimb kinematics.

Ex-vivo Micro-CT Scanning of Hindlimb Bone

Following termination, all femora and tibiae were removed for ex-vivo micro-CT scanning using a custom scanner (150/225-Ffi-HR-CT, BIR, Lincolnshire, IL) that includes a $225 \mathrm{KV} \mathrm{X-ray} \mathrm{source} \mathrm{with} \mathrm{a} \mathrm{focal} \mathrm{spot} \mathrm{size} \mathrm{of} 5$ $\mu \mathrm{m}$ and an image intensifier with a $1024 \times 1024$ pixel digital camera (ACTIS, BIR, Lincolnshire, IL) (Voor et al., 2012). The basic X-ray power settings were $86 \mathrm{kV}$ and $110 \mu \mathrm{A}$. Scans represent a full $360^{\circ}$-plus-fan-angle rotation, with 1000 projections per revolution covering a $3 \mathrm{~mm}$ distance below the knee joint. Two complete rotations covering 214 slices were performed for each scan. A custom MATLAB program (v6.5, The Mathworks, Matick, MA) was used to isolate the compact bone from the less dense cancellous or trabecular bone, and to calculate the bone volume fraction (BV/TV) of the distal femora and proximal tibiae bilaterally. 


\section{Electrophysiological Assessment}

To assess ankle flexor and extensor tone and spasticity we performed bilateral electromyographic (EMG) recordings of LG and TA (heteronomous muscles) at rest and during tail pinches for all animals at weeks 2 and 8 as previously described (Chapter 2 methods). Briefly, animals were held securely with a cloth stockinette on a wooden platform so the hindlimbs were suspended and accessible for fine wire electrode placement. In order to confirm proper electrode placement, pROM was performed by a handler to show that muscle groups bursted in isolation when stretched. We then allowed the animals to settle, and recorded 1.5-minute periods during which no stimulus or touch was given. Following the at rest recordings a rubber clamp was used to pinch the tail in a series of 5 times to elicit bursts of movement responses in the hindlimbs. The percent bilateral co-contraction was calculated as an indication of spasticity. The duration of both muscles firing divided by the duration of either muscle firing per set of bursts was calculated and converted to a percentage. The absolute duration of elicited bursts is represented in seconds.

Histology of Spinal Cord Injury Epicenter

All animals were euthanized at week 8.5 with excess anesthetic (pentobarbital, $110 \mathrm{mg} / \mathrm{mL}$ i.p.) and perfused transcardially with $4 \%$ paraformaldehyde and calcium free tyrodes. Spinal cord segments T7 through L6 were removed for histological processing and analysis as previously described (Hadi et al., 2000, Magnuson et al., 2005, Chapter 2 methods). Briefly, adjacent sets of spinal cord slides were stained for white (eriochrome cyanine) or gray 
matter (cresyl echt violet for Nissl bodies) and processed for spared white matter at the epicenter. Every $5^{\text {th }}$ section from the epicenter was photographed so the total area of white matter, healthy gray matter, unhealthy gray matter, cavity and total area could identified and traced using a Wacom Intuos (Vancouver, WA) drawing tablet. Traced images were opened in ImageJ (NIH software) for the area calculation of each tissue region as previously described (Chapter 2 methods).

Statistical Analysis

All data are presented as group means with standard deviations $( \pm S D)$. Outcome measures were analyzed using repeated measures analysis of variance (ANOVA) with groups as a factor, and were followed by Tukey's HSD and Bonferroni post hoc t-tests when appropriate. Group differences were considered statistically significant for at least $p \leq .05$. The binomial proportions test was used when appropriate, no corrections to the proportions were required.

\section{RESULTS}

Behavioral Assessment Recovery of Hindlimb Locomotion

Our prerequisite goal of mitigating severe pathological ankle contracture in the current study was achieved. In contrast to our previous study in which a daily hindlimb muscle stretch protocol was incorporated, but had no effect on preventing ankle contracture in WC animals, in the current study we did not include stretching and purposely designed the wheelchair to allow more movement of the hindlimbs. Passive ROM of the ankle joint was assessed by 
manual goniometer measurements and revealed significant loss of pROM for WC animals during weeks 2 and 6 (WC group averages: $108.0 \pm 12.55$ at week 2 , $112.5 \pm 15.91$ at week 6 , vs. non-WC: $135.0 \pm 0.00$ at all weeks, $p<.05$ ) that resolved by week 8 (WC $123.0 \pm 12.55$ week 8 ) such that no differences were evident from non-WC animals. It should be noted hypomobility was only evident in the flexion end point values, rather than extension, of the ankle joint for WC animals.

Beginning at week 2.5 the BBB scores for the WC group were significantly lower than non-WC and remained lower throughout the remainder of the 8 week study, (Figure 8D). The dashed horizontal line represents occasional weightsupported plantar stepping $(B B B=10)$, so while the loss of function for the WC group is not as severe as in our previous study, the separation of groups above and below this milestone of locomotor recovery is physiologically relevant. In support of this distinction we plotted the response rate of BBB subscores for the groups, and significant differences are evident for weeks 2, 4, and 6 (Figure 8E). Qualification for a BBB subscore is dependent upon consistent weight support, regardless of whether the steps are dorsal or plantar placed. At day 4 (week 0.5) all animals, as expected, were dragging their hindlimbs with no weight support and had varying degrees of hindlimb joint movement. No animal, regardless of group, had a BBB score that indicated the ability to produce any plantar weightsupported steps at 1 week (Figure 8D), therefore both groups have a response rate of 0 for the BBB subscore at weeks 0.5 and 1 (Figure 8E). Between weeks 1- 2.5 the non-WC animals recovered consistent weight support, and therefore all 
received a BBB subscore. From week 2.5 and later non-WC animals had consistent weight support and frequent/consistent plantar stepping, and was their maximal locomotor recovery throughout the remainder of the study. As a group, the WC animals recovered consistent weight support only after the $24 / 7$ in wheelchairs stopped, after week 6 during the 2 weeks of in-cage activity, so that $80 \%$ received a BBB subscore. However, the stepping was frequently dorsal, therefore the scores never reached a BBB score of 11 . These data indicate a task-specific delay in recovery of locomotor function for WC animals.

Kinematic Assessment of Intralimb Coordination During Overground Stepping There were significant differences in overground locomotion between groups due to the lack of plantar stepping, as measured by BBB scoring, for WC animals. The relationship between joint angles and trajectory of hindlimbs during overground stepping during side view kinematic analysis did not take into account whether stepping was plantar or dorsal, and there were no differences in hip and knee excursion during overground stepping at any time point; weeks 2, 4, \& 6 in wheelchairs, and after 2 weeks of in-cage activity, week 8 (Figure 9). However, the average ankle excursion was significantly different between groups at weeks 4 and 6 for both the peak (maximal extension) and trough (maximal flexion) values, but at week 8 the trough (flexion) values were closer to that of the non-WC controls. These data indicate that WC animals stepped overground with greater extension of the ankle especially during the weeks in wheelchairs.

Swimming Assessment

For this study swimming was used as an assessment of hindlimb pattern that requires no weight support, and was assessed at baseline and terminally 
(Figure 10A,B). We purposely avoided swimming assessments throughout the study to reduce any extra hindlimb afferent input, which may work to train hindlimb trajectory during swimming. Unlike the previous wheelchair study, there were no significant differences in LSS or LSS subscores between the two groups terminally. Kinematic analysis of hindlimb swim cycles, defined as the relationship between the HAT and IHA angles, showed that HAT angles were significantly extended for WC animals (Figure 10C). However, representative ellipses shown in Figure 3D illustrate that the shape and areas of the IHA and HAT relationship angle-angle plots were not different between groups.

Hindlimb Movements While in Wheelchairs

Overall the WC animals recovered a substantial level of hindlimb locomotor function. We were unable to prevent the initial rise in recovery that occurs by 1 week $(B B B=9)$, even though these animals did not experience afferent input associated with in-cage activity. Unlike the previous WC study, we observed varying degrees of ankle, knee and hip movement while the animals were maneuvering about their cages in their wheelchairs (Figure 11). All WC animals presented the hindlimb movements $(n=5$, some more frequently than others) at various times during the 6 weeks in wheelchairs. Figure 4 shows sequential still photos taken from videos of WC animals at week 4 while moving along the length of kinematic assessment tank. Stick figure representations of the right hindlimbs are presented below each animal. In Figure 11A, the animal moves her ankle from against the wheelchair padding (flexed) through to full extension $\left(\sim 150^{\circ}\right)$ twice while moving forward down the tank (red arrows). Figure 11B shows an animal that generates obvious knee movements, while the ankle 
and hip move only slightly. Knee and hip movement in this case is most evident when the black mark on the ankle moves rostrally and becomes hidden by the ankle strap (red arrows). The hindlimb movements were only evident when the animals were pulling themselves forward with the forelimbs, so these movements are loosely associated with the steps on the forelimbs. We were unable to study any relationship of the forelimbs and hindlimbs during these movements because they were random and were not induced by handlers.

Kinematic Assessment During Shallow Water Walking

Shallow water walking (SWW) has been used as both an activity-based locomotor training strategy as well as an assessment tool in our lab. SWW body weight support and lateral stabilization in $5 \mathrm{~cm}$ water for rats in our model. We analyzed IHA and HAT angles for hindlimb trajectory using a side view camera during SWW assessments at weeks $2,4,6$, and 8 (Figure 12). IHA overall excursion was significantly lower for WC animals only at week 6 . HAT overall excursions were significantly lower for WC group during weeks 2,4 , and 6 as compared to non-WC controls, due mainly to significantly higher HAT peak flexion trough values during the same weeks (Figure 12). The IHA angle represents movements through both the hip and knee while HAT angle represents both knee and ankle movements. Interestingly, these data suggest that our IHA and HAT assessments during SWW with $\sim 50 \%$ body weight support may be a more sensitive measure of intralimb coordination in WC animals, perhaps because it involves two joints at a time. This provides $\sim 50 \%$ assessment revealed significant deficits in hip, knee, and ankle movements throughout the 
weeks in wheelchairs that were not evident during overground kinematic analysis.

Interlimb Coordination and Plantar Stepping During Shallow Water Walking We analyzed ventral videos at $2,4,6$, and 8 weeks during SWW for footfall pattern, or interlimb coordination, and plantar paw placement to understand the role of weight support in recovery of locomotion. The CPI and RI measures are significantly lower for WC animals at weeks 2,4 , and 6 as compared to non-WC controls (Figure $13 \mathrm{~A}$ and C). PSI reveals that WC animals were unable to plantar step at weeks 2 and 4 , but all WC animals ( $n=5)$ recovered at least $\sim 50 \%$ hindlimb plantar stepping by week 6 resulting in no differences between groups at 6 and 8 week time points (Figure 13B). These data indicate that WC animals regained hindlimb plantar stepping at some point between 4- 6 weeks, even though they were still in wheelchairs $24 / 7$; yet these animals still had no forelimb-hindlimb coordination at week 6 (Figure 13A CPI and $13 \mathrm{C} \mathrm{RI).} \mathrm{Once} \mathrm{the} \mathrm{animals} \mathrm{were} \mathrm{no} \mathrm{longer} \mathrm{in} \mathrm{wheelchairs,} \mathrm{weeks} 6-8$, there was no dramatic improvement in forelimb-hindlimb coordination as revealed by the PSI scores. Only $n=2$ of the 5 animals showed increases in CPI and RI scores. There was no apparent link between being removed from the wheelchairs for 48 hours on 2 occasions and any measures of gait or locomotion. (see methods for exclusion criteria).

Interlimb Coordination Coupling During Shallow Water Walking We also assessed interlimb coupling using duty cycles of paired limbs. The proportion of the step cycle that homolateral, homologous, and diagonal 
paired limbs were in stance phase together was calculated from underneath camera view during SWW (Figure 14). Phase values for uninjured controls were calculated for comparison (in parenthesis on graph): homolateral and homologous limbs should be out of phase $\sim 0.5$ and $\sim 0.6$ respectively, and diagonal limbs should be in phase $\sim 0.9$. As a whole, coupling phases for WC animals were lower for all three paired limb relationships at weeks 2 and 4 . Specifically, homolateral and homologous coupling was significantly lower at week 2 , and diagonal coupling was significantly lower at week 4 . Similar to the PSI data (Figure 13B), homologous and diagonal coupling (Figure 7A) as well as diagonal distance (Figure 14B) changed dramatically for WC animals between weeks 4 and 6 . Even with the dramatic increase, significant differences were still evident for homologous coupling between the groups at weeks 6 and 8 . These data suggest that gaining hindlimb plantar stepping for WC animals at week 6 is also associated with forelimb-hindlimb coupling phases that approach uninjured values. Interestingly, coupling phases that approach controls at weeks 6 and 8 (Figure 14A) are not associated with better forelimb-hindlimb coordination as measured by $\mathrm{CPI}$ and $\mathrm{RI}$ at the same weeks (Figure $13 \mathrm{~A}$ and $\mathrm{C}$ ).

EMG Recordings of Muscles Controlling the Ankle

At weeks 2 and 8 , we assessed all animals for co-contraction (as a surrogate of spasticity) of hindlimb ankle extensor and flexor (LG and TA, respectively) muscles to understand if chronic unloading of the hindlimbs contributed to the inability for WC animals to achieve consistent plantar stepping (Figure 15). Electrophysiological assessment consisted of several 1.5-minute 
recordings of a series of brief tail pinches that consistently elicited visually obvious hindlimb responses. Additionally, we assessed spontaneous bursting when animals were at rest with no stimulation from handlers. Figure 15A shows the percentage of co-contraction of bilateral LG/TA muscles at rest and during tail pinches revealing that non-WC animals had significantly greater co-contraction than WC animals during tail pinch at both 2 and 8 weeks, but no significant differences were found at rest. The burst duration was significantly longer for WC animals when we compared bouts of activity in the ankle muscles while the animals were at rest.

Ex-vivo Micro-CT Scanning of Hindlimb Bone We used ex-vivo Micro-CT scanning to assess bone loss and cancellous bone turnover in the proximal tibiae and distal femora bilaterally (Figure 16). There were no significant differences between groups for cancellous and cortical bone volumes in tibiae and femora. Consistent with previous studies in our lab (Voor 2012) of $12.5 \mathrm{~g}-\mathrm{cm}$ T9 injuries, tibiae cortical bone volume fraction $\sim 0.55$ and cancellous bone volume fraction was $\sim 0.25$ (Figure $16 \mathrm{~A}$ and $\mathrm{B}$ ).

Body, Muscle Weight and Histological Assessment Body weights were not significantly different between groups at time points assessed (Table 2). At terminal week 8 hindlimb muscles LG/MG, bicep femoris, and quadriceps were significantly lighter for WC animals as compared to non-WC controls (Table 2). There was decreased spared white matter at the injury epicenter for non-WC group, $\sim 11$ vs. $\sim 17.5 \mathrm{~mm}^{2}$, which approached significant difference between groups, $p=.056$. 


\section{DISCUSSION}

Task-specificity and Locomotor Recovery

Just as task-specific improvements in functional recovery (stepping,

standing, swimming) can be trained, we show here that a task-specific delay in recovery is evident following a moderate $\mathrm{SCl}$ in which animals routinely have substantial functional recovery. During daily handling and while in wheelchairs $24 / 7$, plantar paw surface and weight support afferent input components of locomotor recovery were prevented. However, WC animals showed the ability to generate stereotypic movements (not quantified) of their hindlimb joints while in their wheelchairs (qualitatively described in Figure 11). Nichols' laboratory has shown that localized feedback of proprioceptive afferent input is necessary for proper intralimb coordination during stepping and postural stability (Nichols et al., 1999; Abelew et al., 2000), so avoiding the severe ankle contracture we observed in the previous WC study was a primary goal. Indeed, WC animals recovered overground intralimb coordination quite well, similar to the non-WC group, within 2 weeks (Figure 9). Even though flexor and extensor joint endpoints are not accessed during uninjured normal stepping, a moderate $(\sim 20 \%)$ loss of PROM in ankle flexion may have contributed to the observed deficits in ankle flexion (trough) values during the swing phase in later weeks (4 and 6) for WC animals (Figure 9). WC animals also exhibited greater ankle extension peak values. Our kinematic analysis does not separate ankle extension at toe off from extension at toe down, but taken together our data indicate that stance phase 
distance may have been shorter and the distal most portion of limb (ankle to toe segment) was oriented more caudally with reference to the hip when stepping. Our data for ankle extension peak values is inconsistent with the group average ankle kinematic data presented in Canu et al. model of hindlimb unloading (HU) for two weeks. The authors found greater flexion at toe off and toe down for HU animals as compared to controls, however they mention that a few $\mathrm{HU}$ animals showed hyper-extension at toe off (2009). Our data indicate that chronic hindlimb unloading was not a major factor in the kinematics of overground stepping.

Alternatively, although the evidence is limited and far from conclusive, our data is suggestive that the ankle ROM, $\sim 90-150^{\circ}$ (Figure 4), and related proprioception from the repeated hindlimb movements while the animals were in wheelchairs effectively maintained or trained, albeit with some deficits in ankle excursion, intralimb coordination.

There were short periods during which WC animals experienced load and plantar afferent input during the 6 weeks of wheelchair housing. After each daily pressure relief, massage, and skin care routine the animals were allowed to rest in standard cages for at least 1 hour. Importantly, the WC animals spent most of that daily hour of pressure relief in standard cages grooming, and activity in the form of moving around the cage was rare. We tried to avoid afferent input and possible training effects just due to activity during assessments by only performing SWW and BBB assessments every other week, and eliminated swimming throughout the study except at baseline and terminal time points. These opportunities for load and cutaneous afferent input represent chances for 
training locomotion early after injury, and may have contributed to the notable dramatic recovery for WC animals' plantar placement of the hindlimbs between weeks 4 and 6 ( $n=5$ out of 5 WC animals increased score PSI, Figure 13B) even though they were still in wheelchairs $24 \pi$. Certainly, WC animals had in-cage activity between 6 and 8 weeks in standard cages with the combination of intralimb coordination, hindlimb loading, and recently recovered plantar placement, so improvements were expected over that period. While we are confident that extensor load and plantar cutaneous components were largely prevented we cannot yet quantify the incremental amounts, or intensity, of these afferent inputs. It has been established that the greater the number of steps taken, by an order of magnitude, can positively affect stepping kinematics for transected animals trained on a treadmill (Cha et al., 2007; de Leon et al., 2011). If in-cage activity is related to recovery of locomotor function, then one might equate the conditions of natural recovery for incompletely injured animals in standard cages as the best combination of early afferent components for optimal recovery. Quantified measurement of the amount and components of in-cage activity is forth coming.

Training vs. Maintenance Injury severity and location are limiting factors for functional locomotor recovery. The issue of training versus maintenance was discussed in the SWW study from our lab. As noted in Kuerzi et al., there may be a separation of the recovery of pattern formation as occurring early and spontaneously, but may only be evident if a certain degree of weight support is recovered (or provided) as well (2010). Only non-WC animals recovered interlimb (forelimb-hindlimb) 
coordination (CPI $\sim 60 \%$ ) and plantar stepping (PSI $\sim 50 \%$, Figure 13) with partial weight support in SWW. It is reasonable to assume that plantar paw placement is concomitant with the ability to support body weight during stepping. However, it is a distinct possibility that the WC animals were capable of supporting substantial amounts of body weight during weeks of virtually exclusive dorsal stepping (weeks 2 and 4). Furthermore, hindlimb kinematics during overground stepping suggests that WC animals were actually supporting their weight, otherwise the peak angles achieved by extensor muscle groups controlling the hip and knee would have been significantly lower in WC controls. As shown in other studies (Harkema et al., 1997; Timoszyk et al., 2005) the afferent input associated with limb loading during the assessment may have contributed to intralimb coordination in WC animals so they were not different from non-WC controls about the hip and knee. Since $\sim 50 \%$ body weight is provided during SWW there was less required extensor activation during the stance phase that may have negatively influenced intralimb kinematics for WC animals during SWW.

Therefore one might conclude that assessment (not training) in shallow water for animals already capable of frequent/consistent weight-supported stepping overground is not an indicator of recovery since extensor limb loading was not optimal during this task. This interpretation does not contradict the BBB scores indicating a lack of consistent weight support in WC animals because BBB scoring considers the cumulative stepping abilities throughout an entire 4-minute assessment period. On the other hand, kinematic analysis samples the 3 best stepping passes an animal achieved, at a minimum. We do not analyze passes 
in which the animal falls or stops; therefore it is an assessment of the best of what the animal can do. We will need to study hip height or other measures of weight support during overground stepping more carefully in future studies. Furthermore, we will need to incorporate measures of forelimb-hindlimb coordination during overground stepping, not just during SWW.

The CPG is capable of producing near-normal intralimb coordination/hindlimb trajectory kinematics and plantar stepping within a few weeks post-injury when partial body weight support is provided (Heng and de Leon, 2008; Courtine et al., 2009; Kuerzi et al., 2010). Other studies using more severe injuries have shown that after 6 weeks the fundamental locomotor ability of intralimb coordination/hindlimb trajectory kinematics/pattern generation begins to decrease, and training is required to maintain this ability out to several weeks or even months post-injury (de Leon et al., 1999a; Heng and de Leon, 2008; Courtine et al., 2009; Kuerzi et al., 2010). However, in the current study both intralimb kinematics (Figure 12) and plantar stepping (Figure 13B PSI) were maintained in our non-WC control animals, likely due to the less severe moderate $12.5 \mathrm{~g}-\mathrm{cm}$ contusion. Interestingly, there was a decrease in SWW indices involving forelimb-hindlimb coordination from week 6-8 (Figure 13A CPI and 13C $\mathrm{RI})$, indicating that finer aspects locomotor function congruent with the less severe injury are also vulnerable to decline as the time post-injury passes without training intervention. On the other hand WC animals were prevented from incage training that contributed to or maintained plantar stepping, and forelimbhindlimb coordination for non-WC animals very early within the first several 
weeks post-injury. The balancing act of training lost locomotor functions with maintaining intrinsic CPG pattern generation is a complex interactive process in which both function to either limit or amplify potential for locomotor recovery longterm. If $50 \mathrm{~g}-\mathrm{cm}$ severely injured animals were capable of producing and maintaining intralimb pattern, via hindlimb movements while in wheelchairs, our model may be able to maintain that capability for a wider window of plasticity so the specific component of weight support could be trained to optimize functional locomotor recovery long-term.

Chronic Unloading

The deficit in locomotor recovery in WC animals is not likely due poor health since their body weight was not different from controls at the time points we measured. Granted, there was a significant loss of muscle mass for WC animals likely due to prolonged unloading of hindlimbs (Table 2). Hindlimb unloading $(\mathrm{HU})$ has been studied extensively in animal models of hindlimb disuse by orthopedic immobilization via casting, suspension using a body harness, and tail suspension (Morey-Holton and Globus, 2002). Decreased mass and crosssectional area, torque production, peak tetanic force, and increased rate of shortening are common consequences of chronic unloading (Edgerton and Roy, 1994; Edgerton et al., 2002; Shah et al., 2006), but neuromuscular rehabilitation in the form of step training with body weight support (Stevens et al., 2006; Jayaraman et al., 2008; Liu et al., 2008b) or passive cycling (Roy et al., 1998) can mitigate these losses. Neural adaptations to unloading include potentiation of sensory feedback, more motor units arerequired to achieve a given motor task and resultant increase in fatigability (Edgerton et al., 2002), and overall 
decreased excitability of motor neurons (Cormery et al., 2005). While muscle atrophy may seem substantial in more sever models of disuse (space flight or denervation), the loss of maximal peak force in $\mathrm{HU}$ and suspension models is not substantial enough to evoke gross changes in locomotion such losing the ability to support body weight (Roy et al., 1991). Prior to 2012 our wheelchair model of hindlimb immobilization presented in Chapter 2 was one of very few animal studies that combined both hindlimb muscle unloading and clinically relevant incomplete SCl; Castro et al. used complete transection and the Vandenborne laboratory has recently published a metabolic study of hindlimb muscle after joint restriction limb casting with incomplete SCI (Castro et al., 1999; Ye et al., 2012). Additional studies using models that closely represent the combined effects of $\mathrm{SCl}$ with unloading are needed to better understand the intensity (short bouts of high intensity, intermittent, or long-term aerobic) and qualities (weight-bearing standing, passive cyclic) of afferents needed to maintain muscle. WC animals muscle weight was $\sim 25 \%$ lighter than controls in the load bearing ankle and knee extensor muscle groups (Table 3); however ankle flexor muscle TA was not subject to muscle loss in WC animals. We do not know how much muscle loss was evident at weeks during $24 / 7$ wheelchair use, so it is possible that muscle atrophy was more severe at weeks 4 or 6 , but partially recovered during the 2 weeks of in-cage activity weeks 6-8. As discussed above, the loss of $20 \%$ of ankle flexion passive ROM indicates a loss of extensibility of ankle extensors, but active flexion of the ankle is determined by TA activation, so both of these muscle groups may have contributed to suboptimal overground hindlimb intralimb 
coordination of the ankle in WC animals during weeks 4 and 6 (Figure 2).

Terminal muscle mass was reported in both WC studies, however mass and cross-sectional area do not always explain the loss of muscle strength (Berg et al., 1997; Deschenes et al., 2002) indicating a neural component. Therefore, we cannot assume that the muscle loss observed in WC animals had absolutely no adverse affect on locomotion, and repeated measures of specific anatomical and physiological outcome measures in muscles are needed in future studies.

As reviewed by Battaglino (2012) and Dolbow (2011) studies on temporal profile of lower extremity bone loss after $\mathrm{SCl}$ have shown that rapid loss of bone density occurs within days or weeks, up to $33 \%$ within the first few months and up to $50 \%$ out to three years post-injury. Furthermore, severe bone loss due to $\mathrm{SCl}$ can be partially reversed but the benefits are not easily maintained when loading and/or FES stimulation interventions were used, and as a whole data from these types of studies are quite variable (Dolbow et al., 2011). SpragueDawley rats with lower thoracic transection experienced $28-40 \%$ loss in bone mineral density in the epiphyses of femora and tibiae at 6 months post-injury (Jiang et al., 2007). A study lead by our colleagues in the Voor laboratory found that rats with clinically relevant contusion $\mathrm{SCl}$ that were able to obtain the functional threshold of occasional weight-supported stepping were able to mitigate bone loss (2012). In our current study there was no significant bone loss of femora and tibiae of WC animals for cortical and cancellous bone volume, even though their hindlimbs were unloaded for 6 weeks (Figure 16A and B). In accordance with data on patients (discussed above Battaglino and Dolbow) if 
bone loss was going to occur we would have seen it our model by 6 weeks postinjury. This data supports the notion that even though the WC animals were largely prevented from achieving extensor loading they were none-the-less capable of supporting their weight. Sympathetic activation via repeated episodes of autonomic dysreflexia have been shown to induce bone loss after $\mathrm{SCl}$, so it is possible that our low thoracic moderate contusion $\mathrm{SCl}$ is not severe enough to induce these neurogenic components of bone loss (Zaidi, 2005; Jiang et al., 2006; Morse et al., 2011; Masi, 2012). Unlike Liu and colleagues (2008a) we did not find a positive correlation of gastrocnemius mass and BV/TV in the tibiae indicating that muscle mass suffered greatly due to chronic unloading but bone volume did not. Therefore, some other factor contributed to the lack of bone loss that was expected for the WC animals in that proprioceptive movement achieved by the hindlimbs while in wheelchairs may represent loads sufficient enough to preserve bone structure. Indeed, Rubin and colleagues have shown that low intensity, high frequency oscillations of muscle activation invoke anabolic processes in bone (2001). The bone data from our novel model of locomotor recovery in the hindlimbs suggests a low intensity intervention that emphasizes proprioception may mitigate bone loss in patients.

Drawbacks of the current study are low group numbers ( $n=5$ per group) and lower spared white matter for the non-WC group that approached significance ( $p=.056$ independent T-test with equal variance assumed) (Table 2). We do not posit that 6 weeks of $24 / 7$ in wheelchairs somehow spared white matter damage; rather an untoward happenstance left us with more severe $\mathrm{SCl}$ 
in our control group even though we randomly assigned animals to each group prior to the moderate $12.5 \mathrm{~g}-\mathrm{cm}$ injury. No spared white matter values in the current study were outside the normal distribution $( \pm 3 S D)$, and variance in this measure in our previous studies ranges are comparable ( $\sim 3-6 \%$ Smith et al., 2009, 2- 4\% Kuerzi et al., 2010, 3.5- 5\% Table 2), indicating the NYU system has a consistent degree of variability in our hands. An additional drawback is that the co-contraction results (Figure 15A) are unexpected; we assumed chronic unloading would have contributed to greater spasticity in opposing ankle muscles TA and LG. On the contrary, WC animals in this study had less co-contraction in ankle muscles at both time point assessed, weeks 2 and 8 (Figure 15). These data may indicate that WC animals' ability to move the hindlimbs without hindlimb loading while in wheelchairs may have reduced co-contraction, but this is not supported in the literature. It is possible that WC animals would have had the expected higher co-contraction if the EMG assessments had been performed at weeks 4 and 6 since these weeks revealed the most significant deficits in overground stepping and walking in shallow water for WC animals, which were not evident at the earlier 2 week time point. Furthermore, $\sim 80-90 \%$ co-contraction in non-WC animals that were able to perform frequent-consistent weightsupported stepping, and had near-normal ankle excursion kinematic while stepping overground (Figure 9), may not seem feasible. We are not aware of such a high percent co-contraction of opposing muscles in the literature. The degree of spasticity in our model may not be comparable to other studies, and is $10-20 \%$ higher than observed in the WC animals in the previous study. It is most 
likely that our methods of EMG recording are not ideal because we are not assessing activation of ankle muscles while the rats are performing a motor task that requires alternation. During EMG assessments the hindlimbs were suspended while the tail was pinched. The movement response is often air stepping, a cyclic activation across all or some of the joints of the hindlimb, however the response is not consistent. It is possible that we were agitating the animals during the tail pinch assessments and a generalized activation of musculature was the prominent response. We plan to assess EMG activity in the hindlimbs during functional locomotor activities like stepping or swimming to better assess spasticity in future studies.

Conclusion

In conclusion, we have shown that after a moderate low thoracic contusion animals that usually recover considerable functional locomotor recovery can be task specifically delayed due to lack of specific afferent components. Preventing plantar paw placement and weight support by placing animals in wheelchairs for 24 hours per day/7 days per week limits consistent plantar weight-supported stepping overground and forelimb-hindlimb coordination while walking in shallow water. Proprioceptive afferent input was not prevented and may have contributed to intralimb coordination evident very early, 2 weeks, post-injury. Furthermore, just as specific components of afferent input can be exploited during locomotor training to achieve incremental improvements in locomotion we have shown that locomotor capabilities can be parsed and become vulnerable to loss of function. These data support the hypothesis that animals with low thoracic moderate contusion $\mathrm{SCl}$ achieve maximal locomotor 
recovery via in-cage activity. Our two related wheelchair studies taken together suggest that the necessary means of functioning in daily life for $\mathrm{SCl}$ patients, mobility via a wheelchair, is detrimental to lower limb function and limits the potential for adaptive plasticity long-term. 
Figure 8. BBB scores WC 24/7.

A. Animals were in wheelchairs that allowed hindlimb joints to move through an open ROM 24 hours per day $/ 7$ days per week, for 6 weeks. B. Shown are kinematic representation of static positioning of the right hindlimb (thick red lines) with possible excursions of hindlimb segments (lighter colored red lines). C. Passive ROM was assessed weekly using a manual goniometer placed on bony landmarks as identified by manual palpation. D. Overground stepping recovery as assessed by the BBB reveals that WC animals had significantly lower BBB scores (could not produce consistent weight supported plantar steps at any point). However, WC animals did recover dorsal weight supported stepping upon removal from wheelchairs weeks $6-8$. E. The proportion of animals per group that qualified for BBB subscore was significantly different between groups. $\left({ }^{*}, n=5 / 5, \pm S D, p \leq .05\right)$.
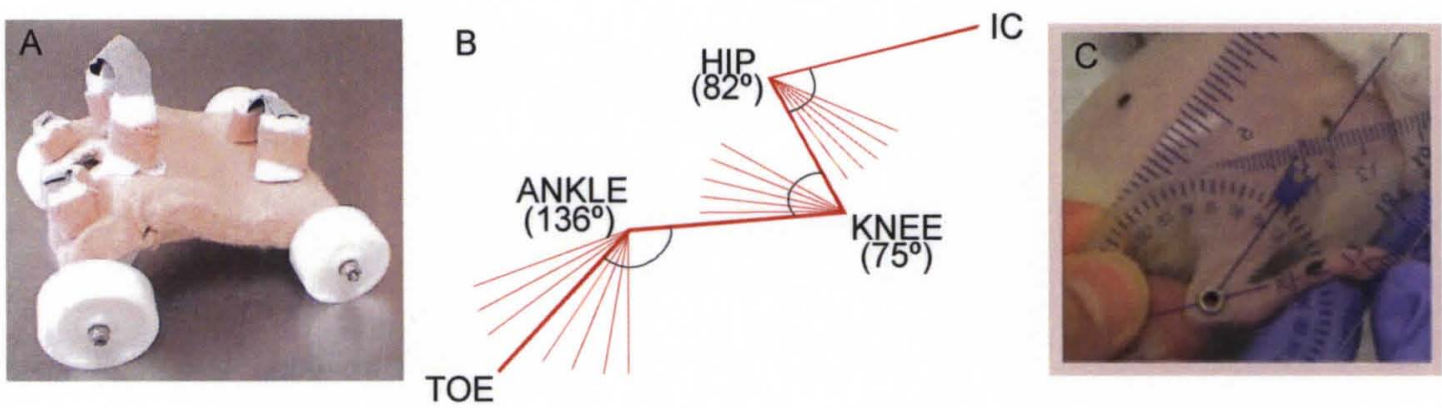

D

E
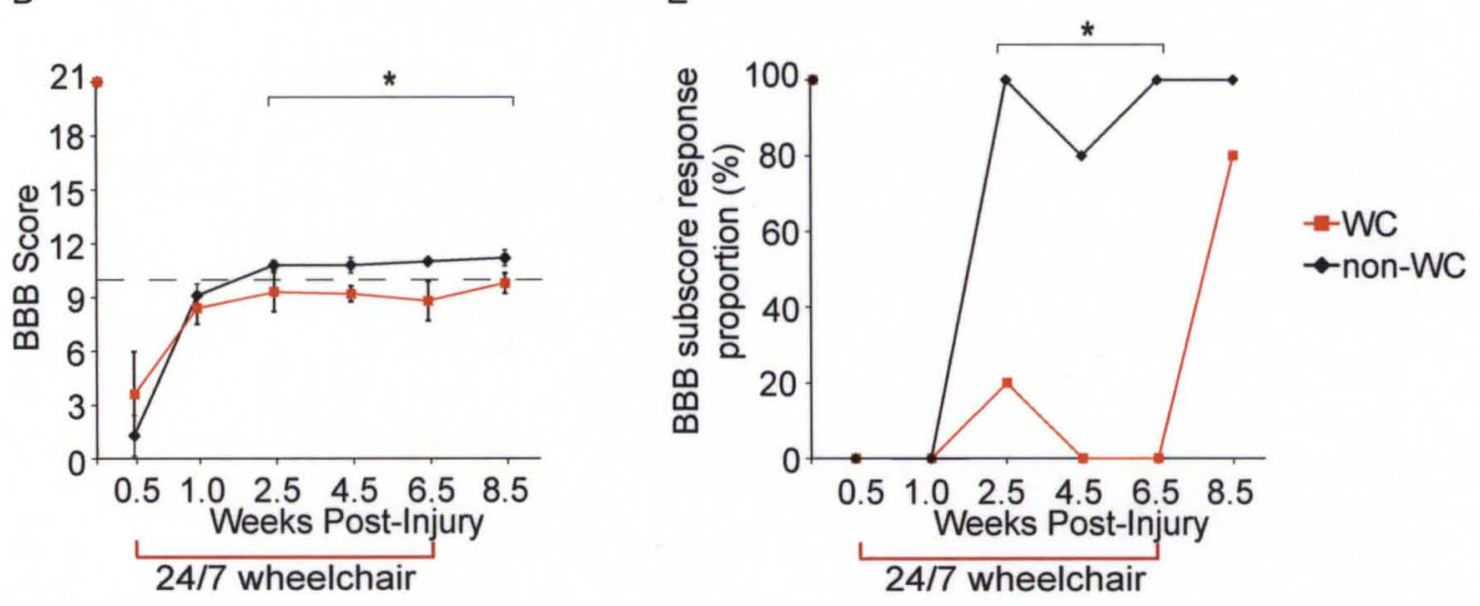
-WC non-WC

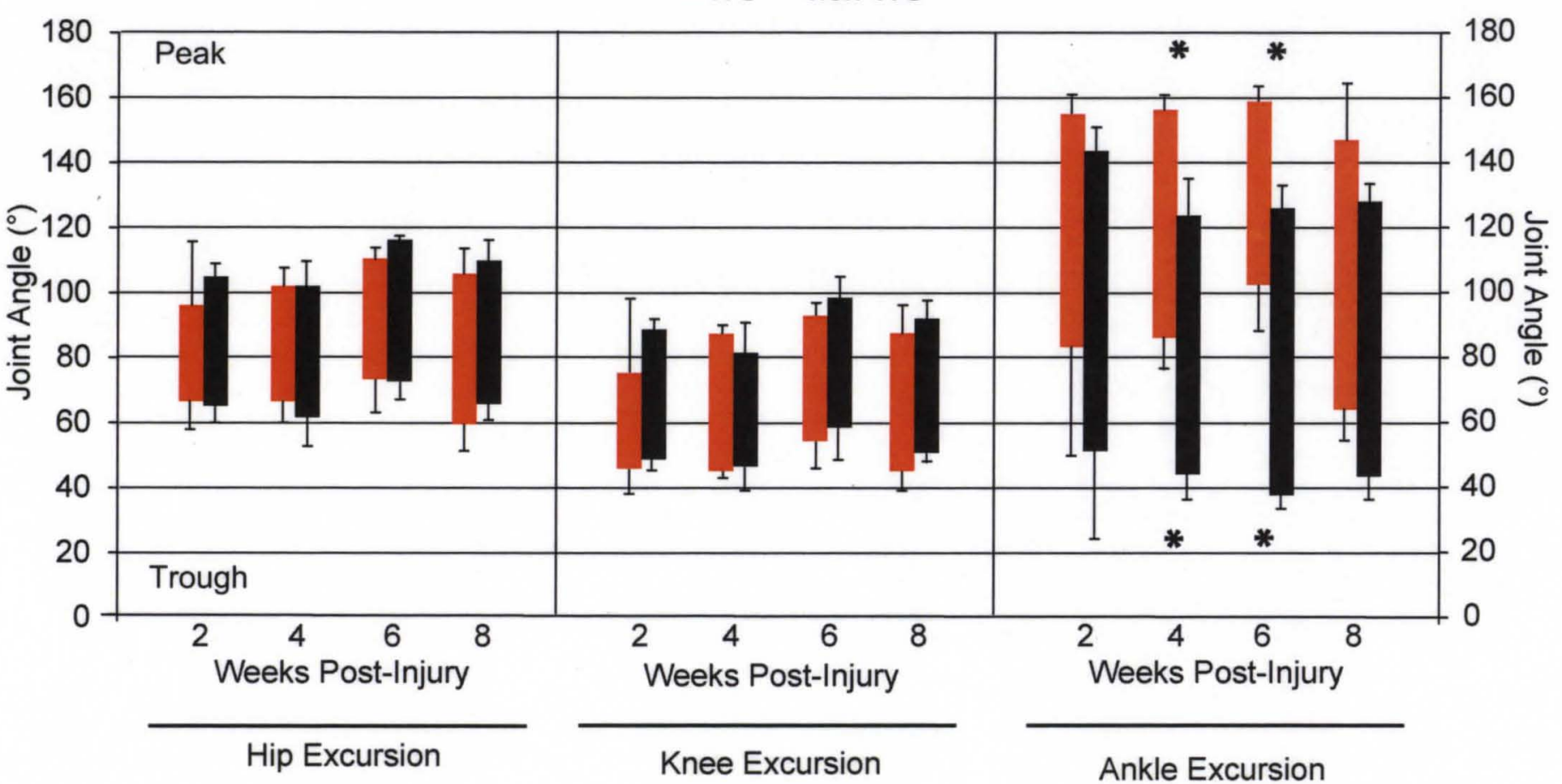

Figure 9. Intralimb Coordination Overground Stepping WC 24/7.

WC and non-WC groups show no difference in hip and knee joint excursion while stepping overground throughout the duration of the 8 week study. Ankle excursion showed significantly greater extension (peak) and less flexion (trough) only during weeks 4 and 6. WC animals show transient compensation of intralimb coordination only about the ankle $\left({ }^{*}, n=5 / 5, \pm S D, p \leq .05\right)$. 
Figure 10. Swimming Assessments WC 24/7.

A and B. Swimming ability, as assessed by the LSS and LSS subscore, showed no significant differences between WC and non-WC controls. C. Kinematic analysis of hindlimbs during swimming revealed that the HAT angle was significantly more extended through its excursion $\left({ }^{*}, n=5 / 5, \pm S D, p \leq .05\right)$. D. Representative examples of angle-angle ellipses from a single swim cycle show no significant differences in the shape and area (inset) between groups.

A

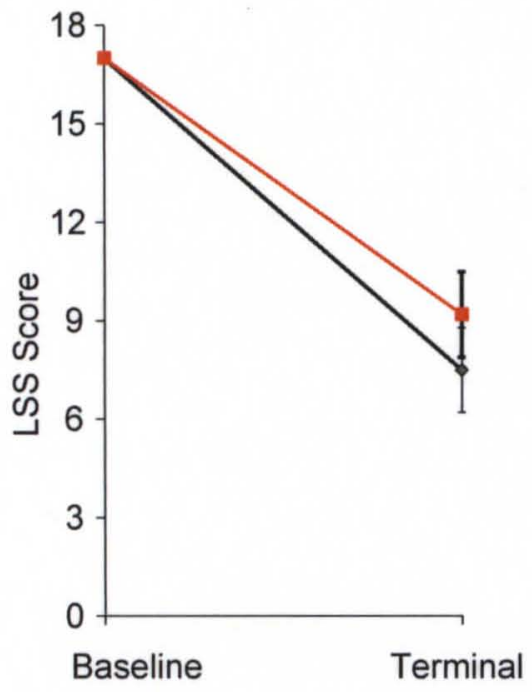

B

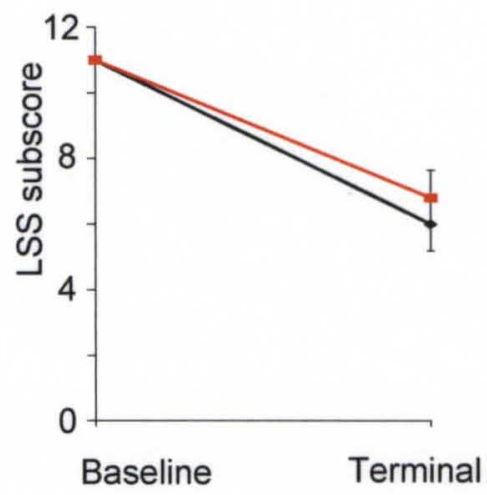

$\because \mathrm{WC}$ non-WC
C

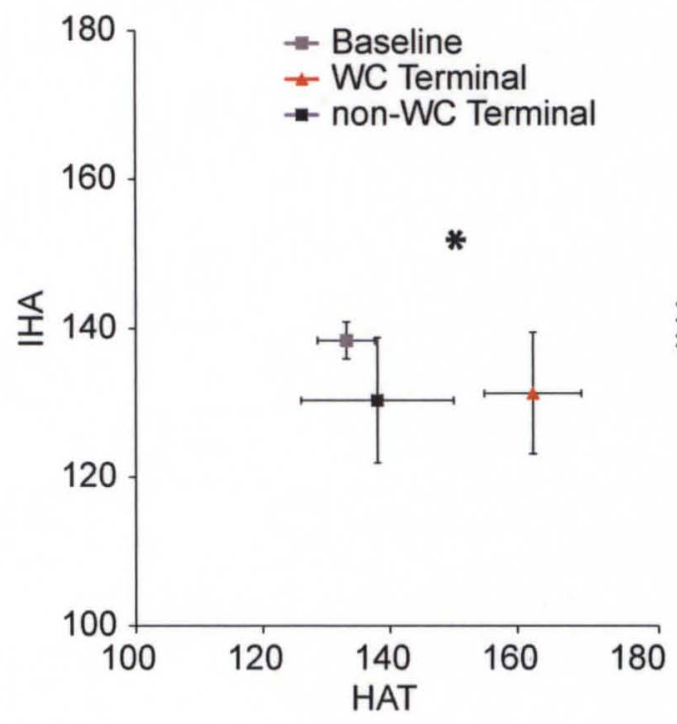

D

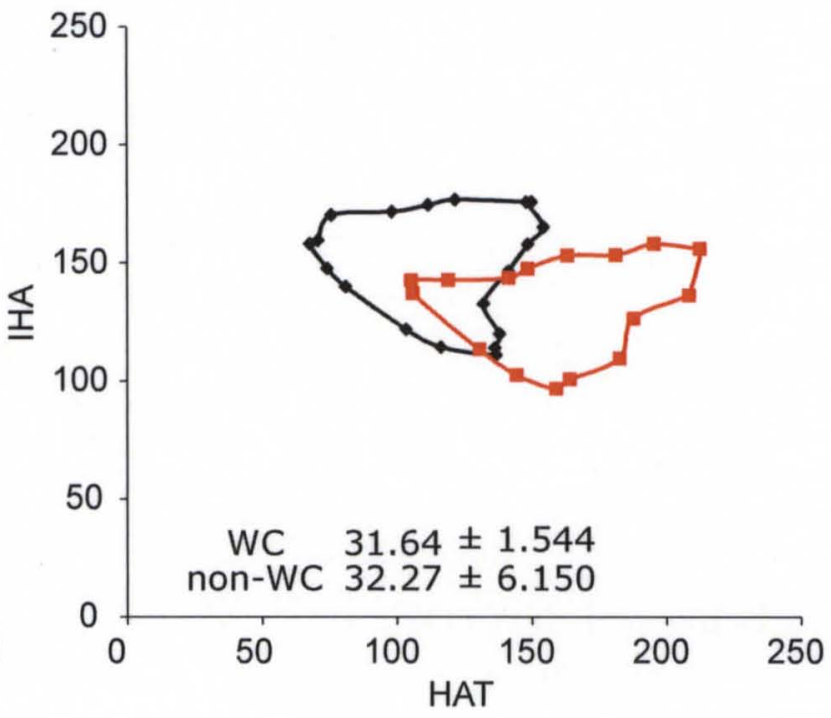


A
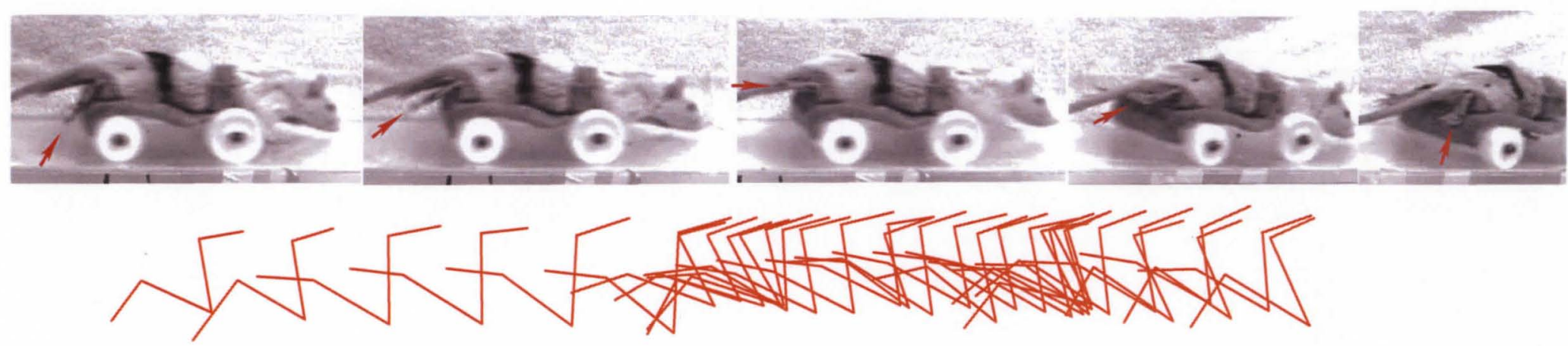

B
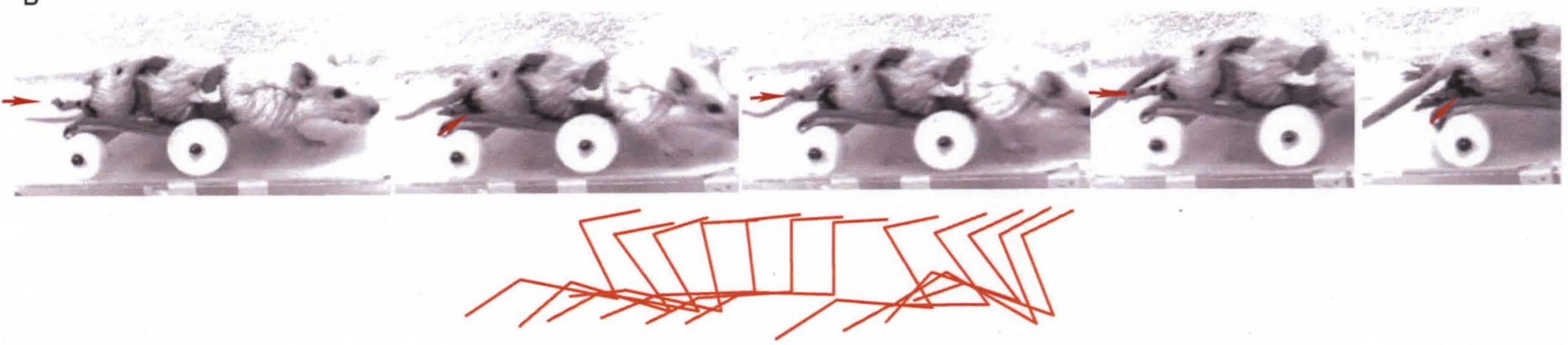

Figure 11. Hindlimb Movements While in WC 24/7.

Proprioceptive input from hindlimbs may have contributed to locomotor recovery for the WC group. A and B. Shown is a series of still photos derived from side camera videos of hindlimb movements from 2 representative WC animals. Stick figures from kinematic analysis illustrate the movements. Hindlimb movement while in wheelchairs was random and episodic, no stimulation was required, and varying degrees of movement through the hip, knee (B) and ankle (A) were observed. 
Figure 12. Intralimb Coordination Shallow Water Walking WC 24/7.

Overall excursion of the IHA angle was significantly lower for WC animals at week 6 as compared to non-WC controls (left side of graph). HAT overall excursions were also significantly less for WC animals (right side of graph) at weeks 2,4 , and 6, due mainly to significant deficits in maximal flexion (trough) values $\left({ }^{*}, n=5 / 5, \pm S D, p \leq .05\right)$.

A

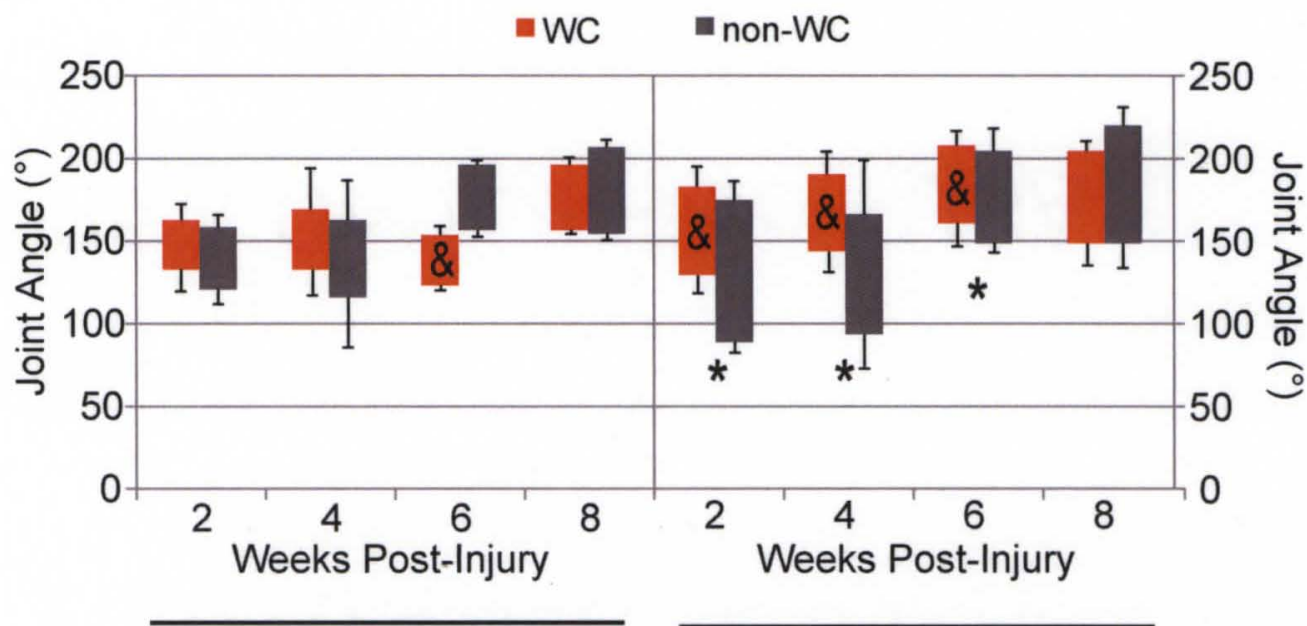

IHA Excursion

HAT Excursion 
Figure 13. Pattern Formation and Plantar Stepping During Shallow Water Walking WC 24/7. $\mathrm{A}$ and $\mathrm{C}$. CPI and RI measures for correct pattern of footfall sequences are significantly lower for WC animals as compared to controls for weeks 2,4 , and $6\left({ }^{*}, \pm S D, n=5 / 5, p \leq .05\right)$. B. The ratio of plantar placed hindlimb steps to forelimbs steps (PSI) shows significant delay of recovery for WC animals through week 4, but plantar stepping recovered before week 6 even though animals were still in wheelchairs $24 / 7\left({ }^{*}, n=5 / 5, \pm S D, p \leq .05\right)$.
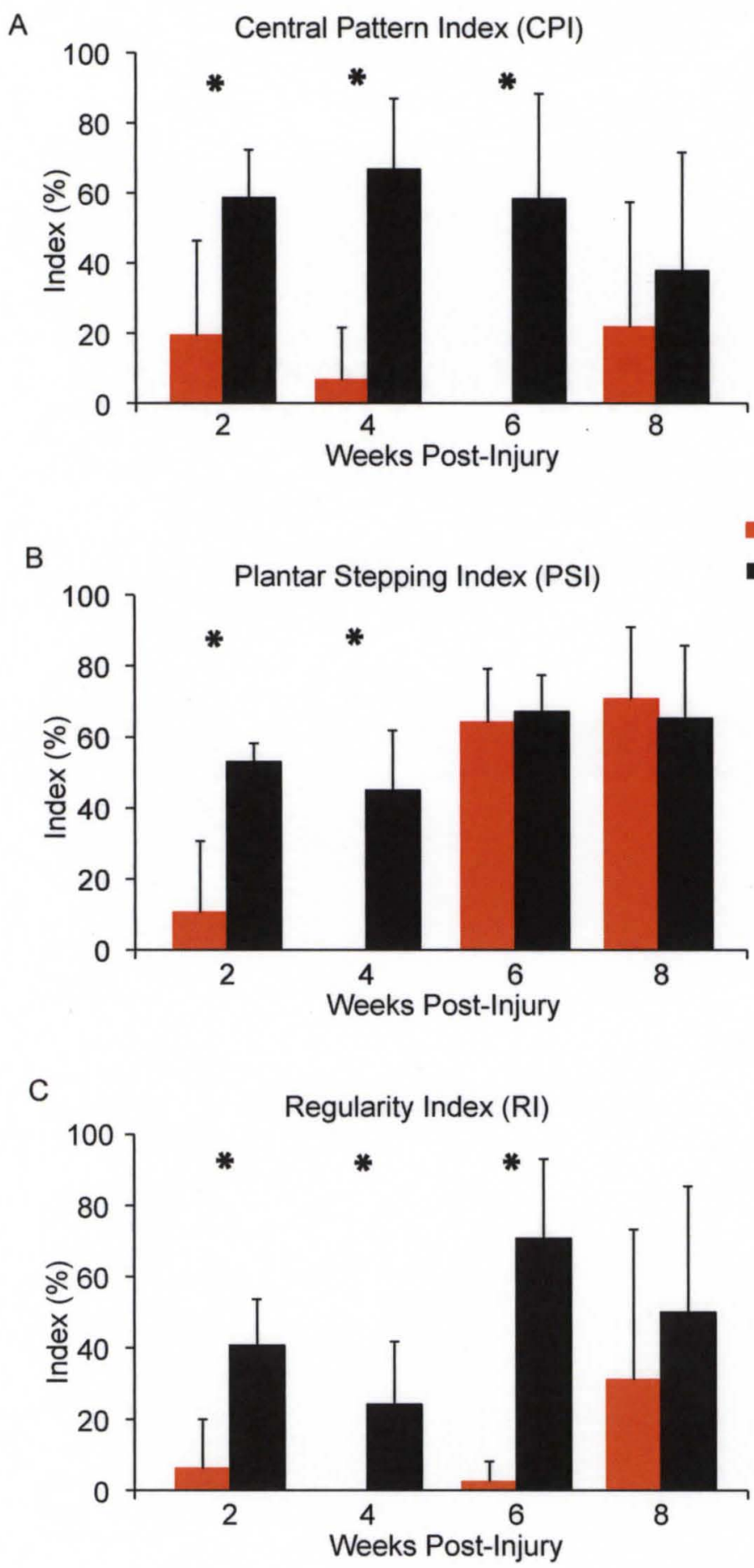
Figure 14. Interlimb Coupling Shallow Water Walking WC 24/7.

A. The stance phase portion of step cycle was evaluated for homolateral, homologous, and diagonal limb pairs. The uninjured normal phase values are in parenthesis below each axis label. WC animals have a significantly lower proportion of step cycle in which paired limbs are in phase: Homolateral coupling is significantly lower at week 2, homologous coupling is significantly lower at weeks 2,6 and 8 , and diagonal coupling is significantly lower at week 4 $\left({ }^{*}, n=5 / 5, \pm S D, p \leq .05\right)$. Dramatic increases in homologous and diagonal coupling was evident for WC animals beginning at week 6 (approaching normal). B. Similarly, the distance between diagonally paired limbs during stance was significantly shorter for WC at week 2 , approached significance at week 4, but increased dramatically at week 6 to values close to non-WC controls.

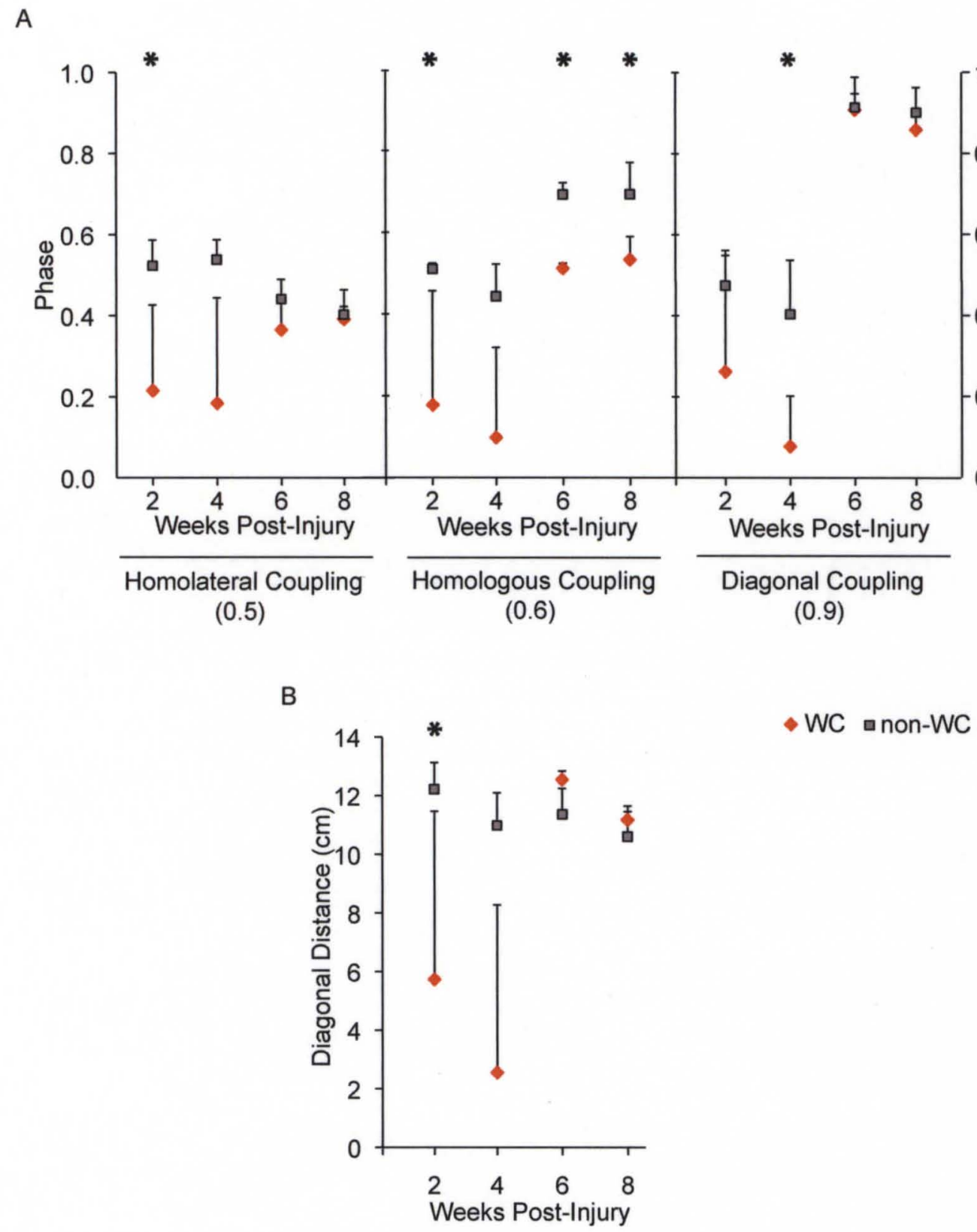


Figure 15. Muscle Co-contraction WC 24/7.

A. The percent co-contraction was calculated for ankle extensor and flexor activation at rest and in response to tail pinch stimulus. WC animals had significantly lower co-contraction during tail pinches as compared to non-WC controls. B. The total duration of spontaneous ankle muscle bursting while animals were at rest, with no stimulus, was significantly greater for WC group as compared to non-WC control ( $\left.{ }^{*}, n=5 / 5, \pm S D, p \leq .05\right)$.

A
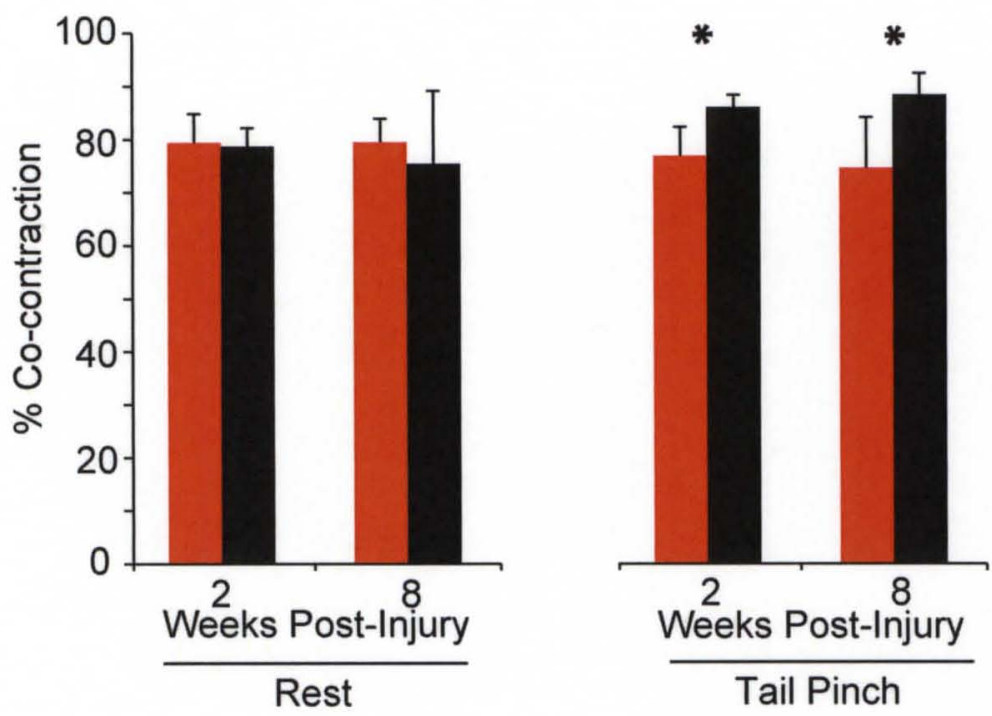

B
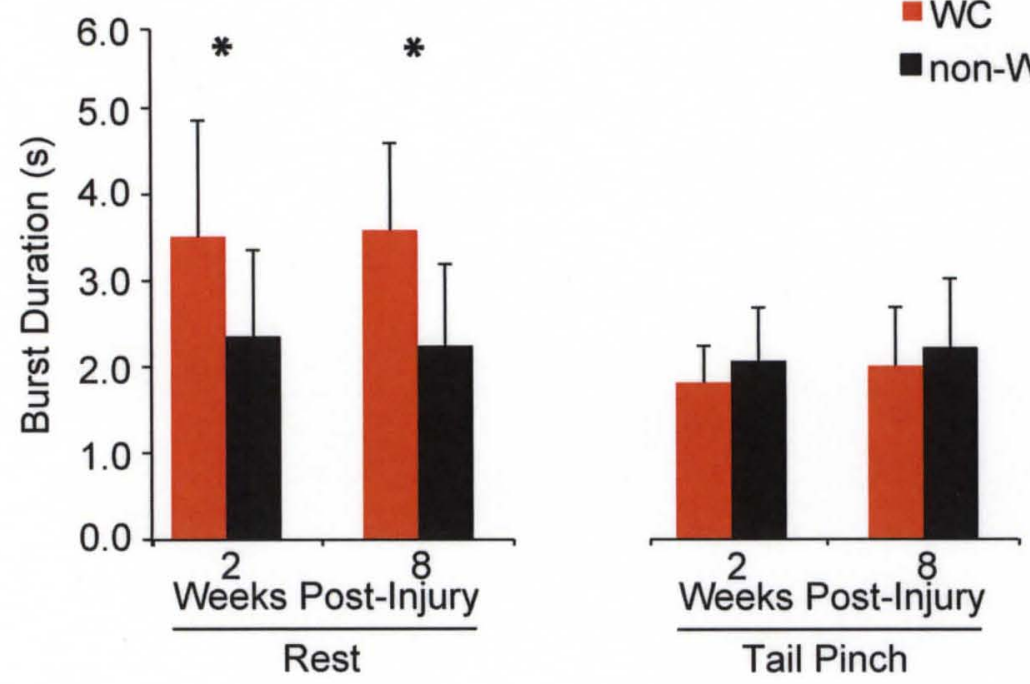

Weeks Post-Injury

Tail Pinch 
Figure 16.. Ex-Vivo Micro-CT Hindlimb Bone Architecture WC 24/7.

$A$ and $B$. No significant differences were evident between groups for cortical and cancellous bone volume fractions.
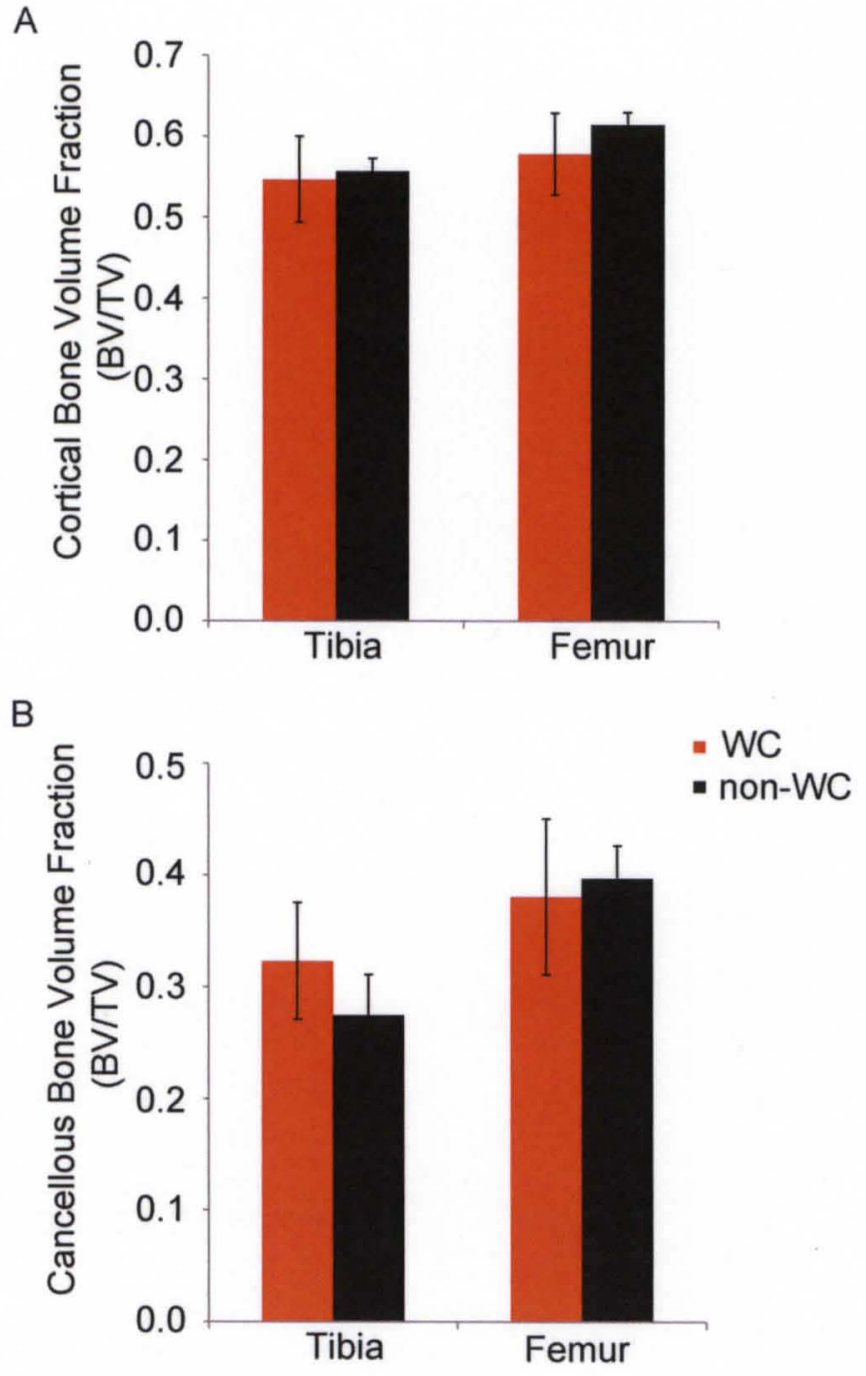
Body Weight (grams).

\begin{tabular}{lccc}
\hline Group & Baseline & wk 4 & wk 8 \\
\hline WC & $203.0 \pm 4.90$ & $201.4 \pm 13.99$ & $245.0 \pm 9.14$ \\
\hline non-WC & $201.0 \pm 8.22$ & $230.8 \pm 12.19$ & $248.8 \pm 14.17$ \\
\hline
\end{tabular}

Hindlimb Muscle Weight Significant Differences (grams).

\begin{tabular}{lccccc}
\hline Group & ankle flexors (TA) & ankle extensors $(L G, M G)$ & knee flexors (pBP) & knee extensors (quad) \\
\hline WC & $0.5024 \pm 0.0692$ & $\star 1.3374 \pm 0.1827$ & $\star 1.6177 \pm 0.1312$ & $\star 1.8107 \pm 0.1317$ \\
\hline non-WC & $0.5215 \pm 0.0627$ & $1.7072 \pm 0.1563$ & $1.8462 \pm 0.1781$ & $2.1322 \pm 0.2277$ \\
\hline
\end{tabular}

Spared White Matter at Injury Epicenter $\left(\mathrm{mm}^{2}\right) \mathrm{p}=.056$.

\begin{tabular}{l|l}
\hline Group & White Matter CSA \\
\hline WC & $17.4 \pm 3.57$ \\
\hline non-WC & $10.9 \pm 4.69$ \\
\hline
\end{tabular}

Table 2. Muscle Weight and Histology WC 24/7.

Hindlimb muscle weights of ankle extensors (LG/MG), knee flexors (BP) and knee extensors (quad) were significantly lighter for the WC group ( ${ }^{*}, n=5 / 5, \pm S D, p s .05$ ), however overall body weight was not different. Differences in spared white matter at the epicenter approached significance ( $p=.056, \sim 17 \%$ SR group, $\sim 11 \%$ non-SR group). 


\section{CHAPTER IV}

\section{THERE IS A NEGATIVE EFFECT OF HINDLIMB STRETCH ON LOCOMOTOR RECOVERY FOLLOWING CONTUSIVE SPINAL CORD INJURY IN THE ADULT RAT}

\section{INTRODUCTION}

Widely accepted standard of care manual therapies for spinal cord injury (SCl) patients have been developed largely in rehabilitation clinics, by physical and occupational therapists and physiatrists, with the goal of preventing further sensorimotor impairment, promoting functional recovery and improving quality of life (O'Sullivan and Schmitz, 2001). Guidelines for manual therapies cite promising preclinical data from animal studies, case studies, anecdotal evidence, and historical references to present the rationale for prescribing these treatments (Harvey et al., 2011). Loss of extensibility of soft tissues spanning a joint including, but not limited to, ligaments, muscles and joint capsules, has been the focus of efforts to maintain function both in peripheral tissues and for optimizing neurological plasticity. Significant health care resources, in the form of stretching and ROM treatments, are allocated in an effort to prevent these issues and maintain joint mobility after $\mathrm{SCl}$. Stretch can be applied by trained therapists or self-administered in the form of manual therapy, splints, serial casts or using intelligent control devices (Zhang et al., 2002; Katalinic et al., 2011). However, as Harvey and colleagues have rigorously analyzed in SCI patient studies, when 
stretch therapy applied for the common regimen of 30 minutes per day for several weeks is subjected to the standards of randomized controlled trials the results show no benefit or the results are inconclusive (Harvey and Herbert, 2002; Harvey et al., 2008; Harvey et al., 2011). The dosage, or duration, of stretch needed to achieve greater joint mobility is also unclear (Harvey et al., 2000; Harvey et al., 2003b; Harvey et al., 2011). As outlined in Ben et al. there is an abundance of animal studies showing a positive effect of stretch on muscle remodeling, sarcomere and collagen arrangement, increased force production and extensibility (2010). However, the authors point out that these findings may not apply to the same degree in patients (a lack of translation), and in many stretch studies extensibility outcomes are measured immediately upon removal of the stretch and initial positive effects are not sustained beyond that immediate time point. This is an important consideration in determining long-term effects that patients seek since contracture management requires lasting increases in tissue extensibility to be effective (Harvey et al., 2011, Katalinic et al., 2012).

$\mathrm{SCl}$ is not the only patient group for which stretch is routinely applied. A review of 25 clinical trials on neurologically impaired patients including stroke, brain injury, $\mathrm{SCl}$, cerebral palsy, muscular dystrophy, and Charcot-Marie-Tooth Disease found little or no short-term or long-term effects of stretch on joint mobility, pain and spasticity (Katalinic et al., 2010; Katalinic et al., 2011). Small but significant increases in ankle dorsiflexion were evident after 5-30 minutes of ankle extensor stretch in individuals with no neurological damage, but the clinical relevance of the findings is questionable (Radford et al., 2006). Increased 
tolerance to stretch, but not improvement in muscle extensibility, is associated with greater ROM of the hip after a stretching regimen in uninjured individuals (Ben and Harvey, 2010). Thus, the dosage required and whether stretch is even efficacious in uninjured subjects is debatable as well. Through more than 10 years of research, researchers have concluded that there is no added benefit from stretch, and methods of effective contracture management need to be reappraised (Katalinic et al., 2011). Harvey (2011) and others (Radford et al., 2006) do not recommend that stretch for patients be discontinued, but encourage further emphasis on evidence-based practices.

As a whole, laboratory animal care in experimental $\mathrm{SCl}$ studies does not usually include traditional stretch or ROM interventions, however the practice simply may not be reported often. A few researchers have noted these efforts in the methods sections on animal care, however testing any effect of these treatments was not the goal of these studies and any influence they had on locomotion was not mentioned (Roy 1992, Hodgson 1994, Ichiyama 2009). Case studies have reported on the use of physical interventions to increase mobility after canine SCl; stretching in the form of traction (Speciale and Fingeroth, 2000), passive ROM, isometric stretch and locomotor training (Smarick et al., 2007) have been used. However, authors admit improvements could not be attributed solely to the physical interventions in those animals. Hypothesis driven studies on the effect of stretch and/or ROM interventions on functional locomotor recovery after $\mathrm{SCl}$ in animal models cannot be found. The lack of supporting data in animal studies and the prolific use of these therapies on SCI patients 
necessitates the investigation of stretch in a translational model.

Our first study included hindlimb stretch of "control" animals that were never wheelchair immobilized (Chapter 2, Caudle et al., 2011). We showed that 8 weeks of a daily bilateral hindlimb stretch negatively influenced the course of recovery for rats mildly contused in the 9th thoracic level (T9) even though they were never in wheelchairs. Group averages for animals that received the stretch protocol revealed an inability to achieve forelimb-hindlimb coordination as measured by the Basso Beattie Bresnahan (BBB) Open Field Locomotor Score $\mathrm{BBB} \leq 14$, a significant deficit as compared to the stepping ability of controls $B B B \leq 18$. Furthermore, the stretch protocol had no influence on the prevention of ankle contracture for animals that were immobilized in wheelchairs during the same 8-week period. These data were surprising to us so we engaged in a second study, not on the effect of stretching on muscle extensibility, but focused on the influence stretching had on preventing the recovery of coordination, a property of stepping exquisitely mediated by the central pattern generator (CPG) for locomotion. We hypothesize that static passive hindlimb stretch, applied early after contusion injury, represents an afferent input stimulus (noxious or aberrant/inappropriate) that confuses or disrupts central pattern generation and functional locomotor recovery. The current study sought to determine whether deficits in stepping would also be evident in a more severe $12.5 \mathrm{~g}-\mathrm{cm}$ T9 contusion injury, in animals that were never wheelchair immobilized. Our data suggests that functional locomotor recovery is significantly limited by only 30 minutes of daily hindlimb stretch in with lower thoracic moderate contusion SCI. 


\section{MATERIALS AND METHODS}

\section{Spinal Cord Injury and Experimental Design}

Nineteen female adult Sprague-Dawley rats $(190-215 \mathrm{~g})$ were used in this

study and all procedures were performed according to the guidelines of the University of Louisville Institutional Animal Care and Use Committee. Animals were randomly assigned into two experimental groups: Acute Stretch (SR), $n=7$, or controls $\mathrm{n}=12$. Each animal was anesthetized with pentobarbital $(55 \mathrm{mg} / \mathrm{kg}$ i.p.) and given prophylactic antibiotics (Gentamicin sulfate $15 \mathrm{mg} / \mathrm{kg} \mathrm{sc}$.) prior to surgery. A single level laminectomy was performed at the T9 vertebrae before receiving a moderate contusion injury $(12.5 \mathrm{~g}-\mathrm{cm})$ performed at the T10 cord level using the NYU Impactor (W. Young, Rutgers University, NJ) as previously described (Magnuson et al. 1999, methods Chapter 3). After injury, wounds were closed in layers using silk sutures with topical antibiotics applied to the incision, and allowed to recover from anesthesia on heating pads. After recovery all animals were housed socially, two per cage, in standard cages for the duration of the study. Cages included Alpha Dry bedding with food and water provided ad libitum. All animals were housed in the same room with 12-hour light/dark cycle and received daily post-operative care, including manual bladder expression as needed. Beginning at post-injury day 4 (Monday, week 0.5 ), the Acute SR group received a daily hindlimb stretch protocol administered each morning, Monday thru Friday, for 8 weeks (Figure 17). Assessments and daily care was continued for another 5 weeks until the terminal time point of 13 weeks. At 10 weeks a 
subset of the original control animals were chosen to undergo 6 days, Monday thru Friday and the following Monday, of the daily stretch protocol (chronic stretch group, $n=6$; Figure 17). All control animals $(n=12)$ were handled daily throughout the study.

Daily Stretch Protocol

Animals were wrapped in terry cloth towels so that the hindlimbs were exposed. The animals were held securely, in a supine position, and it was common to observe rats resting calmly or grooming the terry cloth towel during the stretching. Stretch protocols were administered in a group setting with all handlers present simultaneously. A moderator kept time and monitored finger placement to ensure that each stretch maneuver isolated a single muscle group. The handler to rat pairings were randomized throughout the study. The stretch protocol lasted $\sim 30$ minutes: 1 minute static stretch-and-hold of each major hindlimb muscle group in the order shown in Figure 18A-F bilaterally, for two sets.

The hindlimb stretch positions are shown in Figure 18A-F. Threedimensional (3D) kinematic analysis is derived from the two camera angles shown along the right hand side. The digitized stick figure representations are presented in red along the left hand side. In order to stretch the ankle flexors (tibialis anterior or TA) the ankle was positioned in extension (Figure 18A). Bifunctional muscles include the rectus femoris (assists with both hip flexion and knee extension), the biceps femoris (assists with both hip extension and knee flexion), and the gastrocnemius (assists with both knee flexion and ankle extension); therefore three of the positions required specific positioning of 2 
joints. To stretch the ankle extensors, triceps surae (lateral, medial gastrocnemius and soleus), the ankle was positioned in flexion in combination with knee extension, with care not to stimulate the Achilles tendon with the index finger (Figure 18B). The knee flexors hamstring stretch (posterior biceps femoris and semitendinosis) was achieved by positioning the knee in extension in combination with hip flexion. The knee extensor quadriceps stretch (vastus lateralis, intermedius, medialis and rectus femoris) required the combination of hip extension and knee flexion (Figure 18C,D). In order to stretch the hip adductor muscles (adductors brevis, longus, magnus, minimus, pectineus, gracilis and obturator) hip abduction was achieved by pressing both knees laterally at the same time. Oppositely, hip abductors (gluteus, iliopsoas) muscles were stretched by crossing one hindlimb over the other medially via stabilizing the hips and placing pressure on the thighs/knees (Figure 18E,F). Immediate Stretch Response (ISR) Scores

The intensity, or force applied by the handlers, during each stretch position was monitored carefully by examining the joint angles achieved. The handlers' ultimate goal was to avoid forces that would likely injure the animal. There was a soft end-feel for all stretch positions except for ankle extension, which had a firm capsular end-feel as described by O'Sullivan and Schmitz (2001) and assessed by Darryn Atkinson, PT. All animals had some degree of reflexive and/or volitional responses "Immediate Stretch Responses" (ISRs) during the 1-minute hold of the stretch and/or immediately upon release of the hold. We recorded and tabulated all observed ISRs for each stretch position during every stretch protocol for each animal. Qualitative categories were spasm, 
kick, resistance, airstep, trunk twisting/torsion, writhing of the tail, vocalization and grinding of the teeth. Responses not localized to a hindlimb (trunk torsion, writhing tail, vocalization, and grinding teeth) were always allocated to the ipsiliateral side response; therefore ipsilateral group averages are higher in many cases. As a rule we stretched each muscle group to maximal stretch in which an ISR was elicited but the degree of discomfort was minimized. Handlers judged, on a case-by-case basis, the appropriate intensity based on the end-feel resistance and any ISRs of the animal for a given stretch position. Occasionally a vocalization occurred; in this case the position was adjusted to lessen the intensity of the stretch, and then held for the remainder of the 1 minute for that position.

Goniometer Measurements of Passive ROM

Flexion and extension passive ROM (pROM) of the hip, knee and ankle was assessed using a universal goniometer at baseline and every other week there after. Maximal pROM measurements assess joint mobility without the influence of muscle stretch, therefore extension and flexion angles are greater in pROM than the angles achieved during stretch. Rat hindlimbs have bifunctional muscles crossing more than one joint (see stretching paragraph above); therefore $\mathrm{pROM}$ can vary based on limb position. To assess $\mathrm{pROM}$ the proximal and distal joints on either side of joint being measured must be positioned mid range, and the amount of force applied to the measured joint is virtually none. To ensure that measurements represented maximal flexion and extension for the joint, rather than muscle stretch endpoints, the following general positions were used for PROM measurements: hip extension was measured with the knee 
extended (to prevent stretch of rectus femoris), hip flexion was measured with the knee flexed (to prevent stretch of biceps femoris), knee flexion was measured with the hip flexed (to prevent stretch of rectus femoris), knee extension was measured with the hip extended (to prevent stretch of biceps femoris), ankle dorsiflexion was measured with the knee flexed (to prevent stretch of gastrocnemius). We did not measure pROM of hip adduction or abduction. Total pROM per hindlimb joint was sampled from naïve controls under isoflurane anesthesia to determine the normative values: The hip joint measured $20^{\circ}$ at maximum flexion and $130^{\circ}$ at maximum extension (for notation $20-130^{\circ}$ or $110^{\circ}$ full range), knee $45-150^{\circ}$ (105 full range) and ankle $15-150^{\circ}$ (135 full range). At least 2 examiners measured each joint and many times both examiners helped to stabilize bone segments distal and proximal to the joint assessed in order to prevent multiple joints and structures from moving. In the case of discrepancy the examiners came to an agreement on the final measurements and rounded to the nearest $5^{\circ}$, therefore standard deviations were frequently \pm 0 .

Behavioral and Sensory Assessment

Overground stepping was assessed using the BBB Open Field Locomotor Scale as previously described (Chapter 2 methods). BBB testing began at 4 days (week 0.5 ) on every Monday am (before stretch protocol) and every Monday pm (three hours after stretch protocol) as well as Friday pm (after 5 days of consecutive daily stretch protocol) for 8 weeks, (red bar on $x$ axis, Figure 3A). During the following 5 weeks, after daily stretching stopped, we no longer included the Monday pm BBB testing session. 
Swimming was assessed using the Louisville Swim Scale (LSS) as previously described (Chapter 2 methods) beginning at week 2 and every other week thereafter. Animals in the current study scored within the 0-5 range indicating poor swimming with little or no hindlimb movement, a high dependence on their forelimbs for forward motion, severe trunk instability and/or body angle, or in the intermediate range, $6-11$, with retained dependency on forelimbs for forward movement and with varying degrees of hindlimb movement, trunk instability, and body angle.

Sensory testing was performed at single time points during weeks 5 and 11 to assess hindlimb allodynia or somatosensory hypersensitivity as previously described (Chapter2 methods). In short, animals were arranged one in each of six partitions and allowed to acclimate in each apparatus for $\mathbf{3 0}$ min prior to testing. Both hind paws were sampled five times, with a minimum of two minutes between consecutive samples. At week 5 , the majority of the stretch group animals could not be tested because they were not capable of plantar placement. Regardless of how we prompted the animals to change position or move around the testing surface, there was not sufficient access to the dorsal surface of the paw. At week 11, neither the von Frey test for mechanical allodynia nor the Hargreaves' test for thermal hyperalgesia showed differences between groups.

Kinematic Assessment of Stepping and Shallow Water Walking Kinematic analysis of overground stepping was performed as previously described (Chapter 2 methods). Briefly, hindlimb movements were recorded with two Basler $602 \mathrm{f}$ high-resolution digital cameras running at $60 \mathrm{~Hz}$ connected to a 
PC and using the video software DVR Explorer (Advanced Digital Vision, Natick, MA). Digital AVI files from both cameras were opened in MaxTraq3D (Innovision Systems, Columbiaville, MI) where sharpie marks overlying the iliac crest (I), hip $(H)$, ankle $(A)$ and toe $(T)$ were identified in a semi-automated fashion on each frame. The two dimensional files were digitally combined to estimate a 3D limb position for each frame. Representative examples of stick figures and angle excursion data is derived in Excel or MaxMate (Innovision Systems) and shown in Figure 22. Uninjured normal stepping excursions are referenced within the figure legend.

Digital videos taken with single cameras from the ventral view during shallow water walking (SWW) were processed to calculate three different indices of coordination, the central pattern index (CPI), the regularity index (RI), and the plantar stepping index (PSI) as previously described 2010 . Briefly, the CPI is calculated as the number of correctly patterned cycles (dorsal and plantar cycles) divided by the total number of cycles (dorsal and plantar cycles) regardless of whether the animal can achieve coordination with plantar stepping. The regularity Index (RI), originally described by Hamers and Koopmans (2001 and 2005, respectively), was calculated as the number of correctly patterned planter step cycles/total cycles (dorsal and plantar cycles) and gives an indication of interlimb coordination of plantar only stepping. The PSI is calculated as the number of hindlimb plantar steps/forelimb plantar steps and indicates how consistently the animal is able to achieve plantar stepping.

Magnetic Evoked Potentials from Tail Stimulation 
Magnetically evoked muscle responses (MER) were assessed at 3.5, 6.5 and 9.5 weeks. Each animal was positioned and secured on a piece of wood using a cloth stockinette as previously described (Magnuson 1999). A figure eight magnetic transducer coil placed at the base of the tail was used to directly stimulate afferent nerves resulting in electromyographic responses (EMG, motor output) in hindlimb muscles. Three stimulus amplitudes were used $(60 \%, 70 \%$, and $80 \%$ of maximal output) as previously described (Beaumont 2006). The transducer coil produces a $4.6 \mathrm{~T}$ magnetic field with a $\sim 1 \mathrm{~cm}$ deep by $\sim 1 \mathrm{~cm}$ width peak magnetic field. The transducer coil was positioned by the same research assistant, with care to precisely angle the coil relative to the tail to avoid direct stimulation of the hindlimb muscles and/or the spinal cord. The research assistant was blinded to the groups. EMGs were recorded bilaterally from the lateral gastrocnemius muscles using $26-\mathrm{G}$ needle electrodes connected to Al 405 head stages and a CyberAmp 380 (Axon instruments). Responses were processed for identification of the onset latency, peak-to-peak amplitude and recovery to baseline, and then compared between groups for latency and amplitude.

Histology of Spinal Cord Injury Epicenter Animals were euthanized with excess anesthetic (pentobarbital, 110 $\mathrm{mg} / \mathrm{mL}$ i.p.) at 12 weeks post-injury. The lateral and medial gastrocnemius, and tibialis anterior were blunt dissected free to be weighed and flash frozen before the animal was perfusion fixed. Animals were then perfused transcardially with 4\% paraformaldehyde and calcium free tyrodes. Spinal cords were harvested and prepared for analysis of spared white matter as previously described 
(Magnuson 2005). Briefly, adjacent slide sets containing every fifth section were stained with eriochrome cyanin and Nissl, thus allowing the total area of white matter, healthy gray matter, unhealthy gray matter, cavity and total area to be identified in micrographs and traced using a Wacom Intuos (Vancouver, WA) drawing tablet. Traced images were opened in Image ( NIH software) for the area calculation of each tissue region.

Statistical Analysis

All data are presented as group means with standard deviations $( \pm S D)$. Outcome measures were analyzed using repeated measures analysis of variance (ANOVA) with groups as a factor, and were followed by Tukey's HSD and Bonferroni post hoc t-tests where appropriate. Group differences were considered statistically significant for $p$ values of at least $p \leq .05$. The binomial proportions test was used were appropriate, no corrections to the proportions was required. The nonparametric Wilcoxon signed-rank test was used in the case of ROM measurements in which standard deviations were \pm 0 . Pearson correlations of at least $p \leq .05$ were used to determine the relationships of the Immediate Response Scores (IRS) and BBB Scores.

\section{RESULTS}

Behavioral Assessment Recovery of Hindlimb Locomotion

All BBB scores are shown in Figure 19A and Friday pm only scores are shown in Figure 19B. Unlike the previous studies using WC immobilization, daily hindlimb stretch that began at 4 days prevented the initial rise in recovery that is 
expected for animals with a moderate lower thoracic contusion SCI. Similar to our wheelchair study, the overall profile of recovery features a dramatic deficit in overground stepping in stretched animals through $\sim 4$ weeks, followed by some recovery during weeks $\sim 5-8$ even though the stretch protocol was still being administered daily (Figure 19A,B). Notably, chronic deficits in BBB scores persisted even 5 weeks after the daily stretch protocol ended at week 13 , such that stretching limited recovery to a BBB score of 11 (frequent weight-supported stepping, horizontal solid black line in Figure19A), as compared to the controls that achieved a of BBB $\sim 13$ indicating occasional forelimb-hindlimb coordination and the ability to produce consistent hindlimb stepping. At 10 weeks we chose, based on the tolerance of the animals to a trial of hindlimb stretching (without vocalization), a subset of the original control animals to stretch for 6 days, 5 consecutive days plus an additional Monday ( $n=6$, grey trace, chronic stretch Figure 19C). These animals had lower BBB scores than the remaining animals in the group (controls, black trace $n=6$ ). We separated the data from these groups beginning at week 8 to show that the control animals with BBB> 16 would not tolerate an abbreviated stretch protocol (30-second hold of triceps surae and quadriceps stretches) and were excluded from the chronic stretch group (Figure 19C). We did not compare among groups in Figure 19C. We performed daily BBB assessments on the chronic SR animals (hatched red bar along $x$ axis), and compared daily BBB scores within the chronic SR group. Similar to the acutely stretched animals, the chronic SR group had a saw tooth BBB profile of loss of function. Importantly, after only two days of hindlimb stretch these animals were 
no longer capable of producing weight support $(B B B>9)$. As anticipated, some recovery of BBB scores occurred after the chronic daily stretch ended the next week (Figure 19C).

Interestingly, there is an acute loss of locomotor function for BBB scores on Monday pm (Figure 19A) which would not have been evident if we had only performed BBB assessments weekly, as is customary (Figure 19B). The dramatic loss of locomotor recovery following a single stretching session was first observed on the second Monday of the experiment, week 2, (individual BBB scores are presented in Figure 19D). Animals were only stretched on weekdays; no stretch protocol was administered on weekends and there was a rise in BBB scores from Friday afternoons to Monday mornings (see $x$ axis Figure 19D). The BBB scores show a "saw tooth" functional profile recovery (Figure 19A). Large standard deviations (Figure $3 \mathrm{~A}$ ) exist because not all SR animals were vulnerable to such dramatic loss of function after a single stretch protocol on Mondays (Figure 19D). Indeed one rat had an increase in BBB score by Monday pm (\#72 wk2), and \#58 showed the most consistent scores from Monday am to $\mathrm{pm}$. In order to determine if this loss of locomotor function was due to a specific handler, we plotted the drop in BBB scores for every Monday per handler. No apparent relationship exists for any handler (Figure 19E). Furthermore, there were no significant changes over time for handlers.

\section{Swimming Assessment}

Swimming was used as an assessment of locomotion that does not require the ability to support body weight. Swimming ability was assessed using 
the Louisville Swim Scale (LSS) with supplemental cutaneous feedback (Smith et al., 2006a). Daily SR negatively influences swimming ability during weeks 1-4, the same period that showed the greatest loss of locomotor function (Figure 20A). As a group, the non-SR animals were intermediate swimmers with more hindlimb kicking, greater trunk stability and better body angle (LSS 11) throughout the study. The SR group remained dependent on their forelimbs for forward movement with only occasional hindlimb kicking, severe trunk instability and poor body angle as hallmarks of their swimming during weeks 1-4 (LSS 3). Similar to the BBB profile, LSS scores increased in weeks 4-8 and reached a plateau for the final chronic time points at $10-12$ weeks. We tested the animals for swimming ability on Monday (am and pm) at week 4.5 to determine if a transient loss of function occurs for swimming as in overground locomotion. At that time point only a single point drop in swimming ability was evident (Figure 20A). The LSS scores taken as a whole reveal that the SR protocol appears to negatively influence the recovery of swimming in a manner similar to that of locomotion. Swimming was not significantly affected at week 10 for the chronic stretch group, however we did not test these animals for swimming ability daily (Figure 20B).

Passive ROM of Hindlimb Joints

Passive ROM for flexion and extension of the hip, knee and ankle was assessed at baseline and every even week thereafter (Figure 21). All animals regardless of SR or non-SR group experienced a significant loss of pROM of hip extension $\left(\sim 90^{\circ}\right.$ versus normal hip extension $130^{\circ}$, no group differences existed 
at any time point) up to at least week 4 , but by week 6 pROM of the hip recovered for both groups (Figure 21A) Only SR animals showed a significant loss of $\mathrm{pROM}$ in extension of the knee beginning at 4 weeks which lasted throughout the remainder of the study, even at the 10 and 12 week time points several weeks after the daily SR protocol ended (Figure 21B). No pROM was lost in the flexion values for the hip or knee at any time point for either group. Oppositely, pROM for the SR group in the ankle was characterized by only loss in the flexion values and not for extension, though these differences are not significant (Figure 21C). It is striking that the SR group had loss of pROM beyond that of the non-SR group. These data indicate that the stretch protocol may have induced some degree of inflammation in muscles, ligaments, and other soft tissues that limited pROM; however at no point during the study was inflammation or edema noticeable to handlers and examiners. It is equally plausible that the cumulative loss of locomotor function immediately following hindlimb stretch protocol (Figure 19A) led to an inability for these animals to move about in their cages; they were, in essence, hindlimb immobilized which is associated with loss of locomotor function in our previous studies using immobilization in wheelchairs. Any loss of pROM in the hindlimb joints may contribute to significant differences in intralimb coordination.

Kinematic Assessment of Intralimb Coordination During Overground Stepping Time points were chosen to show overground stepping kinematics within the 8 weeks of daily stretch protocol, week 3 , and several weeks after daily stretch ended, week 13 (Figure 22). Excursions for uninjured normal stepping is 
listed parenthetically within the figure legend for comparison. At week 3, SR animals dragged their hindlimbs with knee contact and very little excursion of any joint (see arrows, Figure 22A). SR animal \#60 exhibited drag with the hip highly extended (grey trace $125^{\circ}$ ), and the knee and ankle held relatively flexed. SR animal \#67 showed a few degrees of excursion about the hip at week 3 (grey trace, Figure 6A). In contrast, at week 3 the non-SR animals stepped with toe down and knees did not touch the surface (Figure 22B). Already at week 3 the non-SR animals had cyclic excursions of all three hindlimb joints (traces \#70 and \#74, Figure 22B), although they were in phase indicating poor intralimb coordination. By week 13 the SR animal \#60 recovered cyclic excursion of the hip of $\left(\sim 40-140^{\circ}\right)$ that was greater than the average excursions for naïve animals (see legend) indicating that the hip excursion may compensate for the lack of knee and ankle excursion that persisted at week 13 (traces, Figure 22C). SR animal \#67 achieved only minimal movements in the knee and ankle, however it did have hip excursion (Figure 22C). The kinematics of overground stepping of non-SR animals did not change drastically from week 3 to week 13 (Figure 22D). These data concur with the BBB scores at 3 weeks in which SR animals were dragging their hindlimbs with movement in no more than 2 joints (BBB 3), meanwhile non-SR animals already exhibited weight-supported stepping (BBB 12), with cyclic excursions of all three joints. By week 13 non-SR \#70 recovered hindlimb joint kinematics of hip, knee, and ankle (traces, Figure 22D) that approached intralimb coordination of higher locomotor function as seen for controls in our previous study (Chapter 2 Figure 3 ). These data indicate there 
may be group differences for intralimb coordination in the current study and group averages for kinematic analysis bilaterally will be helpful to understand how SR affected the kinematics of overground stepping.

Interlimb Coordination and Plantar Stepping During Shallow Water Walking Ventral view videos of the paws during shallow water walking (SWW) allows for assessment of plantar stepping versus dorsal stepping, and whether or not the animal can produce the correct footfall pattern for interlimb coordination when some weight support is provided by the buoyancy of the water (Kuerzi 2010). Group data from weeks 3 and 13 are compared for CPI, PSI, and RI; week 3 is during the 8-week timeframe of daily stretch protocol when BBB scores were very low, while 13 weeks represents several weeks after daily stretch ended and BBB scores had recovered and reached a plateau (Figure 23A-C). Significant differences are evident for all three indices at week 3 since the SR animals were not capable of stepping, and had only minimal movement of hindlimb joints with no weight support. By 13 weeks the ability to produce the correct forelimb-hindlimb coordination with either dorsal or plantar steps had improved for the SR group and was not different from the non-SR group (Figure 23A). Similarly, the SR animals also improved the ratio of plantar stepping in the hindlimbs (Figure 23B). However, the RI was significantly lower for the SR group at the 13-week time point (Figure 23C). The large standard deviation for the RI values for the SR group indicates that at least some of these animals struggled to combine plantar stepping with the finer aspect of forelimb-hindlimb coordination.

Immediate Stretch Responses (ISR) and BBB 
During each stretch protocol the rats exhibited none or any combination of ISRs (spasm, kick, resistance, air stepping, trunk twisting/torsion, writhing of the tail, vocalization and teeth grinding) for each of the static passive stretch positions. A single point was assigned for each response and tabulated for both hindlimbs (Figure 24). There was a significant increase in ISR scores from the first Monday (week 0.5) compared to the remaining Mondays for stretches of the ankle and knee flexors and extensors (Figures $24 \mathrm{~A}$ and $\mathrm{B}$, respectively). The standard deviations for ISRs on the first day of stretch, Monday week 0.5 (4 days post-injury), are very high indicating some SR animals were already showing ISRs very early after injury while others had little or no response. The flexion and extension stretches elicited significantly higher ISR scores than the positions that stretched hip adductors and abductors for all Mondays beginning with the second week (Monday 1.5; Figure 24 compare across A-C). As a whole, ipsilateral responses are greater than contralateral response because non-localized responses, for example vocalizations, were assigned to the ipsilateral side.

The daily stretch protocol occurred immediately after the Monday morning BBB scoring. After stretch, the animals were then placed in their cages for no less than 3 hours and we repeated BBB scoring later on that afternoon. Since there was such a dramatic drop in BBB scores after the stretch protocol (Figure 24D) we wanted to determine if there was a relationship between the ISR score for each stretch position and BBB scores that afternoon. We performed Pearson's correlation analysis for ipsilateral and contralateral group ISR scores each Monday (for each stretch position) with the BBB scores from that afternoon. 
Significant correlations ( $60 \%$ of the data is accounted for) are listed in Table 3: each Monday (weeks), BBB pm scores with the total drop from am to pm in parentheses, which muscle group stretch, which ipsilateral/contralateral ISR was significant, and Pearson $r \& r^{2}$ values. There are only two negative correlations for ISR and pm BBB score; both occurred during the early time point of week 1.5 for ankle flexor stretch ISR and knee extensor stretch ISR (Figure 24E, see Table). This relationship indicates that at week 1.5, when pm BBB scores first indicated a dramatic loss of locomotor function after a single stretch protocol (Figure 24D circled week 1.5), the higher the ISR score the lower the subsequent BBB scores that afternoon. On the other hand, there are several positive correlations of higher ISR scores with pm BBB scores. Interestingly, these positive correlations are present when the BBB scores began to increase from week to week even though the immediate drop from am to pm BBB scores was still evident (Figure 24D circled weeks 4.5 and 5.5). For example, the ISRs from ankle extensor stretch were positively correlated with BBB scores at weeks 4.5 and 5.5 (Table 3). The same knee extensor stretch that showed a negative correlation at week 1.5 had a positive correlation later on at week 5.5 (Figure 24F). Knee flexor stretch also showed a positive correlation for week 5.5 . Importantly, higher ISR scores during these weeks, which are relatively late in the 8 weeks of daily stretch, are correlated with higher BBB scores in the afternoon, even if those pm scores were lower than the am scores. If overstretching had a cumulative effect of damage to the muscles, tendons and/or soft tissues, one would not expect to see positive correlations in the later weeks 
since repeated damage would not be conducive to healing. Our ISR correlations data indicate that overstretching was not likely throughout the study, but that the nervous system became more responsive at later weeks. This suggests that higher ISRs (increased reaction to stretching) were associated with better locomotor function in the last few weeks of the stretching protocol.

Magnetically Evoked Hindlimb Muscle Responses

Magnetic stimulation at the base of the tail evokes a short-latency $(5-6 \mathrm{~ms})$ muscle response, presumably via a monosynaptic reflex, indicating the state of excitability of motor neurons innervating ankle extensors (Figure 25). Responses were induced with three stimulator settings, 60,70 and $80 \%$ of maximum. We assessed evoked responses on Monday afternoons at weeks 3.5, 6.5 and 9.5. Response amplitudes were significantly lower for the SR group only at week 6.5 (Figure 25B). A trend for decreased amplitude for the SR group exists at weeks 3.5 and 9.5 but these are not significantly different between the groups (Figure $25 \mathrm{~A}$ and $\mathrm{C}$ ). At week 6.5, the response amplitude for the SR group is positively correlated with BBB scores from Monday afternoons (Figure 25D) indicating that motor neuron excitability was recovering during week 6.5 . This recovery is consistent with the profile of BBB scores $(\sim 5-8)$ even though the stretch protocol was still administered daily (Figure 19A,B).

Body, Muscle Weight and Histological Assessment Hindlimb muscles were fresh dissected and weighed immediately. Unlike in previous WC studies, ankle extensors LG/MG as well as ankle flexors TA were significantly lighter in SR animals as compared to non-SR controls (Table 4). 
Body weights were not significantly different between groups throughout the study, and there were no significant differences in spared white matter at the epicenter between groups.

\section{DISCUSSION}

Risk of Over Stretch

It is possible that the forces applied during stretch were greatly disproportionate to the biophysical properties of a much smaller animal causing damage by over stretching muscles, tendons, ligaments or other soft tissues. However this problem may be clinically relevant. At least one study has shown the torque applied to stretch the hamstring muscle of $\mathrm{SCl}$ patients varies widely (40-fold) among trained therapists; and uninjured normal subjects would not tolerate the applied forces (30-68Nm) (Harvey et al., 2003a). Hindlimb stretching in our hands may not detect slight changes in end-feel because, barring one key handler, we are not trained physical therapists. However, we believe we did not over stretch for several reasons: 1) Temporary inflammation (edema) can infiltrate peripheral tissues inducing a loss of mobility; there were no visual indications of swelling in the current study. While we cannot rule out the presence of inflammation, any presentation was subclinical and could not be identified visually. 2) The SR animals in our first study with less severe T9 contusions were able to walk throughout the entire experiment and we never observed the severe transient loss of locomotor function; hindlimb stretch only limited the potential for greater functional recovery as compared to non-SR 
controls. 3) During the stretches and immediately upon release of the stretch, either the same leg or the opposite leg often responded with a reflexive or voluntary ISR in both studies. This indicates that the stretching was not causing gross damage to the muscle to the point it precluded the animal from moving the limb immediately. Again, we observed no signs of inflammation that may have accumulated by delayed onset during the hours following and remainder of the day. The inability to walk after the stretch protocol was administered was striking; so we incessantly inspected, manipulated and handled the animals but found no manifestation of peripheral tissue pathology. 4) Vocalizations were only occasional during the stretch protocol, yet these animals would not tolerate the needle puncture through the skin needed for the fine wire implanted EMG analysis at week 3 (cancelled). This indicates that sensory function and pain perception was present when BBB scores were lowest. In short, a 30-minute stretch protocol was relatively tolerable compared to needle stick. 5) Most importantly, the hindlimb muscles of SR animals revealed significant atrophy (Table 4). Stretch-induced hypertrophy is a reasonable result of daily stretch in our model (Khan, 1986; Kelley, 1996). Atrophy in SR animals' hindlimb muscle was likely a result of decreased weight-supported stepping during in-cage activity due to stretch induced loss of locomotor function. Taken together the data produced in our study does not support the notion that we over stretched the hindlimb muscles. Regardless, in future studies we must identify the relationship between applied forces and joint angle (flexion/extension) achieved in order to 
understand what range of forces is appropriate for maximizing stretch but mitigating any risk for over stretching.

Relationship of pROM and Overground Joint Excursion Kinematics The physiological ROM needed to step overground is represented by excursion data for uninjured normal rats during baseline assessments $(\sim 70-$ $115=45^{\circ}$ at the hip, $\sim 45-90=45^{\circ}$ at the knee, and $\sim 40-100=60^{\circ}$ for the ankle); see Figure 3 and Kuerzi et al. (2010). However pROM for the hip joint measured $20^{\circ}$ flexion and $130^{\circ}$ extension ( $110^{\circ}$ full range), knee $45-150^{\circ}\left(105^{\circ}\right.$ full range) and ankle $15-150^{\circ}\left(135^{\circ}\right.$ full range). When compared, the proportion of excursion required for normal overground stepping is less than half of the full pROM for all three hindlimb joints. These differences allow for compensation of joint excursion and trajectory of limb movement during overground stepping observed in our studies (Figures 3, 10, 21, and 22).

During pROM goniometer measurements we apply very little force to the joint's natural resistance indicating the end-range of flexion and extension; therefore the values obtained for pROM may be conservative. Unexpectedly, SR animals showed decreased $\mathrm{pROM}$ in knee extension beginning at week 4 which was somewhat reversed over the following weeks, but remained significantly lower even at the 10 and 12 week time points several weeks after the daily SR protocol ended (Figure 21B). All anatomical components of the neuromuscular system, muscle, joint capsule, ligaments and tendons, are affected during hindlimb stretch. Since pROM indicates mobility of the joint without the influence of muscle tension, our data indicate that inflammation was not outwardly visible still may have contributed to loss of function in the knee joint. Interestingly, loss 
of $\mathrm{pROM}$ in hip extension was evident in both groups after $\mathrm{SCl}$, but resolved in all animals between the 4-6 week time points. Studies on pROM in SCl patients are not abundant, most studies mentioning pROM in the hip do not address it in the context of how it shapes the ability to produce trajectory and joint excursion during locomotor training. However, the inability to extend the hip has weighty consequences on locomotor recovery since hip extension is a reflexive trigger to initiate the swing phase of stepping (Hiebert et al., 1996). Both stretching and cumulative in-cage immobility, as a direct consequence of stretch, are possible causes the reduced $\mathrm{pROM}$ in hip extension. No pROM was lost in the flexion values for the hip or knee at any time point for either group. Opposite of knee and hip where pROM is limited in extension, the ankle was characterized by only loss in the flexion values and not for extension, though these differences are not significant (Figure 21C). Although we did not quantify pROM in our first WC/SR study we can conclude that WC animals that developed contracture of the ankle had severe loss of pROM and WC animals with no contractures and the SR controls likely suffered some varying degree as well. Data on PROM has become a fundamental aspect of assessments in our models. The degree to which loss of pROM encroached upon the ROM required during overground kinematics, as evident in our 24/7 WC animals' ankle flexion, is of concern because it limits proprioceptive afferent input, induces compensatory intralimb joint movements, and trumps potential for greater functional locomotor recovery.

Gain and Loss of Locomotor Function 
The profile of locomotor recovery in the current study of daily hindlimb stretch in animals with moderate contusion is similar to that of 8 weeks of hindlimb immobilization in animals that were only mildly contused (Figure 2). There was a progressive weekly loss of the ability of walk overground through the first 4 weeks followed by gain of locomotor recovery in weeks 4-8 even though daily stretch continued to be administered daily. Daily stretch ended at 8 weeks at which no greater locomotor function was achieved and was significantly lower than control group. The second time course of gain and loss of locomotor function was an unexpected; drastic loss of function immediately following single stretch sessions such that animals were capable of frequent/consistent weightsupported stepping prior to stretch but were flaccidly paralyzed afterwards. Flaccid paralysis persisted for greater than 24 but less than 72 hours.

The similar gain and loss of locomotor recovery in the two studies is suggestive of a common mechanism evident in the two interventions, both of which model standard of care in SCI patients. The flaccid paralysis evident in WC animals and starkly noticeable after a single session of stretching in the current study indicates loss of reflex actions, a classic hallmark of spinal shock. Ditunno and colleagues classified spinal shock in stages including the primary mechanical insult, hemorrhage and ischemia causing areflexia and block of spinal axon conduction, emergence from shock in which reflexes return, and development of hyper-reflexia (Ditunno et al., 2004). During spinal shock there is a fundamental inability for neurons to fire action potentials due to multiple ionic imbalances (Agrawal and Fehlings, 1996), which alter resting potential, and ion 
channel kinetics. The resulting central conduction deficit was described as hyperpolarization by Ditunno et al. (2004); however a state of depolarization is more likely given enhanced $\mathrm{Na}^{+}$channel conductance and copious extracellular $\mathrm{Ca}^{2+}$. Loss of excitatory input from supraspinal centers and decreased descending inhibition of spinal inhibitory pathways also contribute to flaccidity. In response to Ditunno and colleagues overlooking some aspects of spinal shock, Hayes and colleagues succinctly described the role of proinflammatory cytokine tumor necrosis factor alpha (TNF $\alpha$ ) and immune mediator nitric oxygen (NO) in inducing conduction block (2007). Both of these have elevated expression levels in the spinal cord after $\mathrm{SCl}$, and both modulate channel conductance. The severity of conduction block is dependent on the relative increases in these factors, and is restored once the cytokine levels reduce. Oligodendrocyte loss or dysfunction results in demyelination preventing saltatory conduction (Smith et al., 1979) and further complicating neurodegeneration. Spinal shock involves several factors and understanding its resolution in clinically relevant $\mathrm{SCI}$ models is paramount when rehabilitation efforts are increasingly focused on acute time points.

Spinal shock resolves within days, even weeks or months depending on the citation (Ditunno et al., 2004). An activity-based rehabilitation intervention applied very early after injury significantly magnifies secondary injury processes (Griesbach et al., 2004; Smith et al., 2009). In order to avoid this confound but still prevent in-cage training, we began our interventions at about 4 days postinjury when animals begin to move about their cages. James and colleagues 
performed a study characterizing the temporal pattern of conduction block after low thoracic contusion injury in the rat and found that the Ladder Test, rather than overground locomotion, is the proper indicator of the percentage of conduction through the injury and resolution from spinal shock (2011). Therefore, we cannot draw any conclusion on whether animals in our studies were resolved from spinal chock prior to beginning our WC or daily SR interventions. Regardless of whether signs of inflammation and peripheral tissue pathology were (WC hindlimb immobilization, Chapter 2) or were not (current SR study) outwardly visible, our models inevitably induce inflammation and activate c-fibers. Our models seem to reintroduce or prolong spinal shock, likely via a substance $P$ (SP) mechanism from primary afferent response to peripheral tissue inflammation and pain. Dorsal horn neurons show an upregulation of NK1 receptors, both number and distribution, and internalization due to mechanical inflammation such as noxious pinch of the paw indicating that SP plays a role in dorsal circuit reorganization with persistent peripheral inflammation (Abbadie et al., 1997). Moreover, when paw pinch was applied to paws with that already had ongoing inflammation the NK1 expression increased significantly not only in superficial lamina I but also in deep lamina III-IV neurons, and increases were evident beyond the primary terminal region of the paw afferents in L2-L6 spinal segments (Abbadie et al., 1997). NK1 increases are related to not only the intensity of c-fiber activation (Doyle and Hunt, 1999; Adelson et al., 2009) but there is spatial localization such that joint nociception produced NK1 and Fos expression even down into lamina $\mathrm{V}-\mathrm{X}$. Our model of stretch and hindlimb 
immobilization in a WC may not be as intense as other peripheral inflammation models (Freunds Complete Adjuvant), but given that our models affect multiple anatomical components/submodalities of c-fiber activation (skin, joint and muscle) with risk for ongoing (up to 8 weeks intermittently or $24 / 7$ ) inflammation and pain, there is likely a large and widespread influence of SP released from primary neurons on NK1 expressing dorsal horn neurons (superficial and deep) that are in approximation to interneuron regions housing the CPG (Magnuson et al., 1999). Sensitization resulting from noxious afferent input is SP dependent (Ferguson et al., 2006), and excessive stimulation by SP has been shown, in a series of two papers by Baumbauer and colleagues $(2007 \mathrm{~b}, \mathrm{a})$, to diminish plasticity by creating a learning deficit in a model of spinal instrumental learning after transection. Noxious afferent input can also disrupt spinal learning via TNF $\alpha$ activation (Huie et al., 2012) a key pro-inflammatory cytokine and mechanism of immune-mediated central conduction failure after SCI mentioned above (Davies et al., 2006; Hayes et al., 2007). TNF $\alpha$ exerts a decrease, almost extinction, in compound action potential amplitude, depolarization of resting potential in a dose dependent and reversible manner (Davies et al., 2006). Furthermore, spinal learning deficit is reversed when TNF $\alpha$ was inhibited (Huie et al., 2012). In essence, spared spinal cord tissue after contusion injury is highly sensitive to peripheral inflammation and pain such that regaining proper neuron firing and conduction is repeatedly interrupted by factors contributing to a state of spinal shock and delayed from functional reorganization.

Stretching and Locomotor Function 
An obvious reason for using stretch is to reduce the risk for musculoskeletal injury due to disuse (Edgerton et al., 2002) and we modelled our protocol after stretching observed in $\mathrm{SCl}$ patients prior locomotor training. However, our data suggests that any and all daily afferent input may contribute to or detract from spinal cord excitability and optimal locomotion. During a National Neurotrauma Society Annual Meeting (2010), Jean Wrathall used the term "conducting plasticity" to describe the interaction of adaptive and maladaptive plasticity. In order to optimize functional recovery we must maintain potential for plasticity, training components of recovery that are not intrinsic to the CPG, and at the same time mitigate the effects of maladaptive plasticity such as pain and minimize exposure to afferent input that does not contribute to functional recovery. WC animals in our first study and SR animals in the third study were not able to in-cage train, or maintain intrinsic locomotor function, because the afferent input they were receiving (muscle stretch of varying intensity and duration) was not functional to locomotor recovery during time spent immobilized. Similarly, animals that are stand trained, lose the ability to step (Edgerton et al., 1997; de Leon et al., 1998; de Leon et al., 1999b) likely due to barrage of extensor load and fusimotor proprioceptive input that is not related to patterned and timed afferent required to optimize locomotion. In reference to long-term plasticity task-specific gain of function coupled with loss of function in other nontrained behaviors may imply that there are certain activities that need to be avoided in order to improve a given function, or "be careful what you train for" (Fouad and Tetzlaff, 2011). These findings support the notion that there may be 
a limited behavioral repertoire that can be trained depending on the amount and location of spared tissue (Tetzlaff et al., 2009).

If an $\mathrm{SCl}$ patient presented the same "saw tooth" transient loss of lower limb function in a clinical setting it might be attributed to generalized loss of peak torque output following stretch (Guissard et al., 2001; Edgerton et al., 2002). However, given the importance of afferent input after $\mathrm{SCl}$ there likely exists a complex interaction of neuromuscular and CPG mediated control of motor output, rather than solely motor fiber consequences. We found brief mention in a primary physical therapy textbook (page 372) that facilitated, "hands-on", therapies such as stretch and $\mathrm{ROM}$ are detrimental for recovery for $\mathrm{SCl}$ patients that are high functioning and capable of independent movements (O'Sullivan and Schmitz, 2001). If this notion is common knowledge for physical therapists, but it is commonly practiced, it would behoove us to continue the studies in animals in order to help refine and reassess the application of stretch as treatment only for patients that truly need it. In addition to the obvious detriments imposed by hindlimb stretch discussed herein, the most relevant implication of our study is that clinical assessments of motor function may be substantially varied and lack true coherence with the 'best achieved' neurological function. This is vitally important because American Spinal Injury Association (ASIA) scores, Functional Independence measure (FIM), and other neurological assessments are not only prognostic indicators, but also dictate the course of treatment. Increased communication, observation, and collaboration among basic science researchers, clinicians, and physical therapists are necessary to optimize 
treatment for SCl patients (Segal et al., 2011). We posit that our data is not surprising to many physical therapists, and that the presentation of such variation of locomotor function may have already been described in their field.

Conclusion

The loss of function due to static passive hindlimb stretch after contusive thoracic $\mathrm{SCI}$ has now been repeated for very mild and moderate injury severities. We have demonstrated that 30 minutes of daily stretch administered during the first 8 weeks after paraplegia results in significant deficits in functional locomotor recovery that persists even 13 weeks post-injury. When a separate group of animals underwent only 6 days of stretching that began in the chronic phase, at 10 weeks, there was a significant decline in BBB scores that was transient, but did not affect locomotion in the following weeks. The negative impact on locomotor function that accompanied daily stretch is likely related to inflammatory and pain mediate exacerbation of secondary injury processes and spinal shock that are hallmarks of SCl. Our results suggest the use of repeated stretch, traditionally used to induce muscle lengthening and extensibility may delay and limit long-term functional locomotor recovery in an animal model of incomplete $\mathrm{SCl}$ that consistently shows substantial recovery by 6 weeks. Our data prompt further investigation on the use of stretch in clinical settings, especially with respect to stretch applied immediately before neurological examination or activity-based rehabilitation strategies. 


duration 13 weeks
handled daily

Figure 17. Timeline 8 Weeks Daily Stretch.

The stretch protocol was administered daily for 8 weeks (red line, $n=7$ acute stretch group) followed by 5 additional weeks of assessments. The timing of BBB assessments, overground stepping kinematic assessments and MER tail responses are noted throughout the 13 week study. A subgroup of control animals was stretched for 6 mornings beginning at 10 weeks (hatched red line, $n=6$, chronic stretch group). 
Figure 18. 3D Kinematic Representation of Hindlimb Stretch Positions.

A- F, Static passive hindlimb stretch was achieved by manual manipulation of the hindlimb joints. Shown is a 3D (two camera views) kinematic representation of the right hindlimb of each stretch position. The anatomical landmarks represented are the iliac crest (IC), hip, knee, ankle and toe.
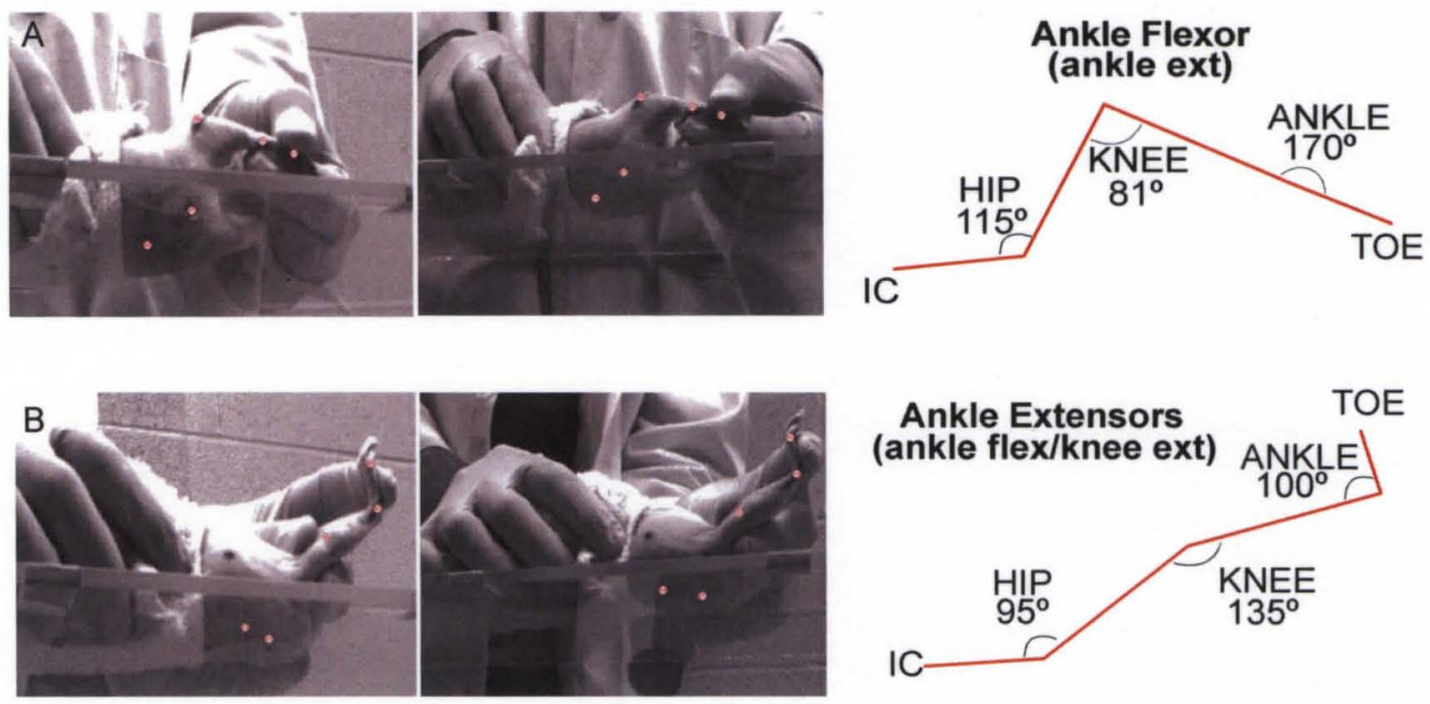
Continued Hindlimb Stretch Positions
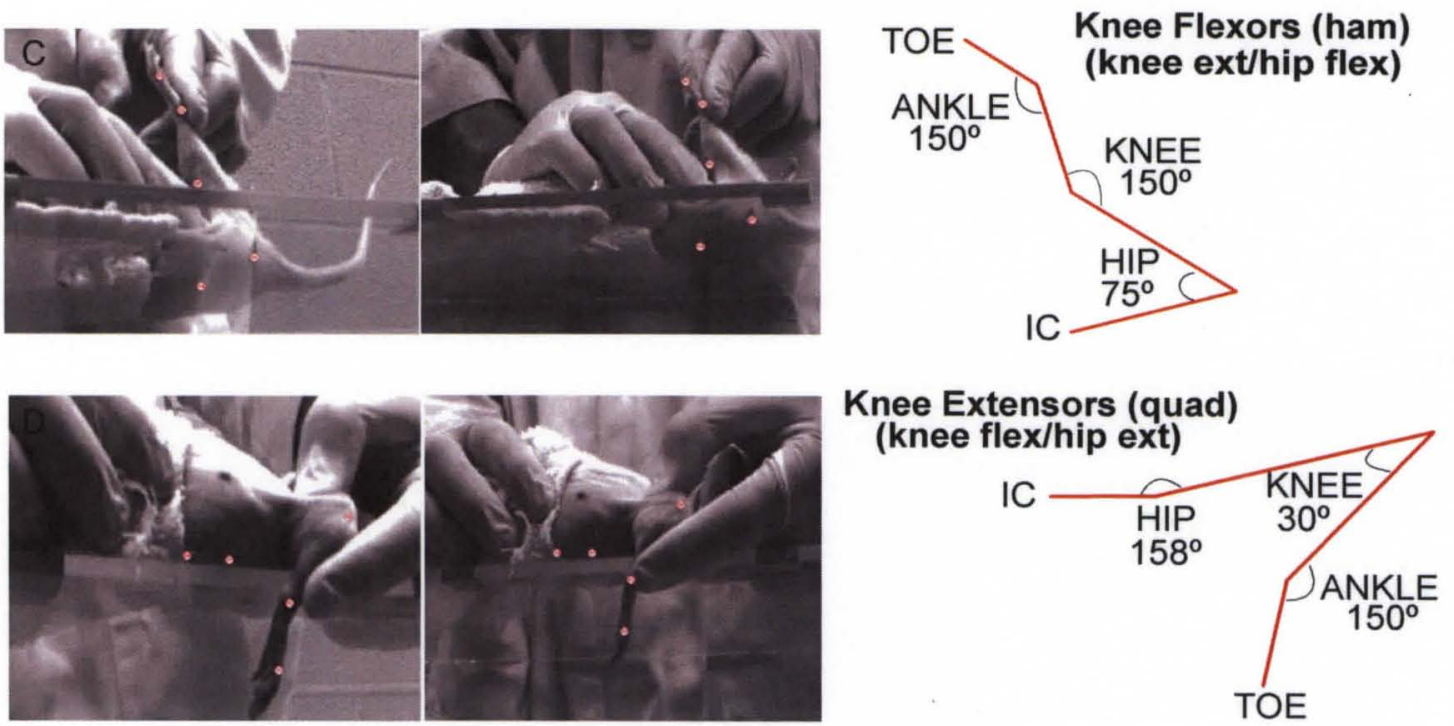
Continued Hindlimb Stretch Positions
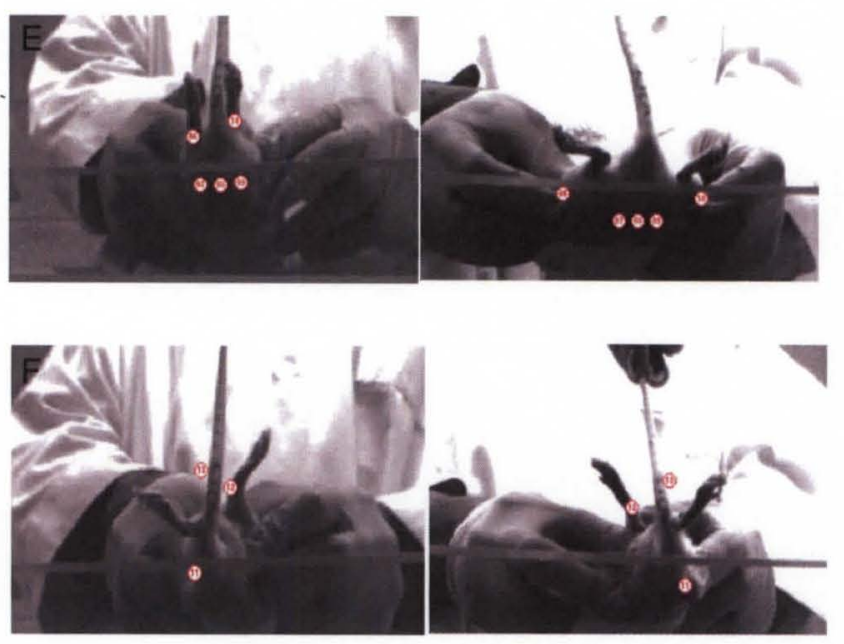

Hip Adductors abduction pre-stretch

$\begin{aligned} \text { HIP } & \text { HIP } \\ 94^{\circ} & 83^{\circ}\end{aligned}$

abduction stretch

${ }_{144^{\circ}}{ }_{156^{\circ}}^{\text {HIIP }}$

Hip Abductors (right) adduction (left)
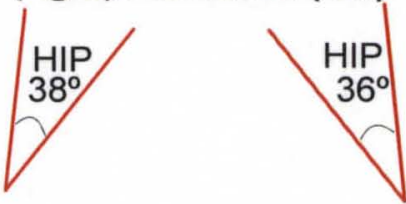
Figure 19. BBB Scores Daily Stretch.

BBB testing was performed three times weekly for all experimental groups (acute stretch (SR), chronic SR, and non-SR controls) Monday am, Monday pm and Friday pm shown in A, only Friday pm shown in B. All animals scored 21 pre-injury. Significantly lower BBB scores were found for the SR group $(n=7)$ as compared to non-SR controls during the 8 weeks of daily hindlimb stretch (red bar $x$ axis) as well as at chronic time points beyond 10 weeks. Animals receiving the daily SR protocol showed dramatic but transient loss of locomotor function until $\sim 5$ weeks after which BBB scores gradually increased (weeks 5-8) and reached a plateau $(B B B=11)$ at 9 weeks after daily stretch ended. Figure $3 \mathrm{C}$ shows the chronic SR group that received the stretch protocol daily for 6 days at 10 weeks (grey trace). Significant loss of function for the chronic SR group occurred after only two consecutive days of stretching, as well as immediately following SR on the following Monday morning.
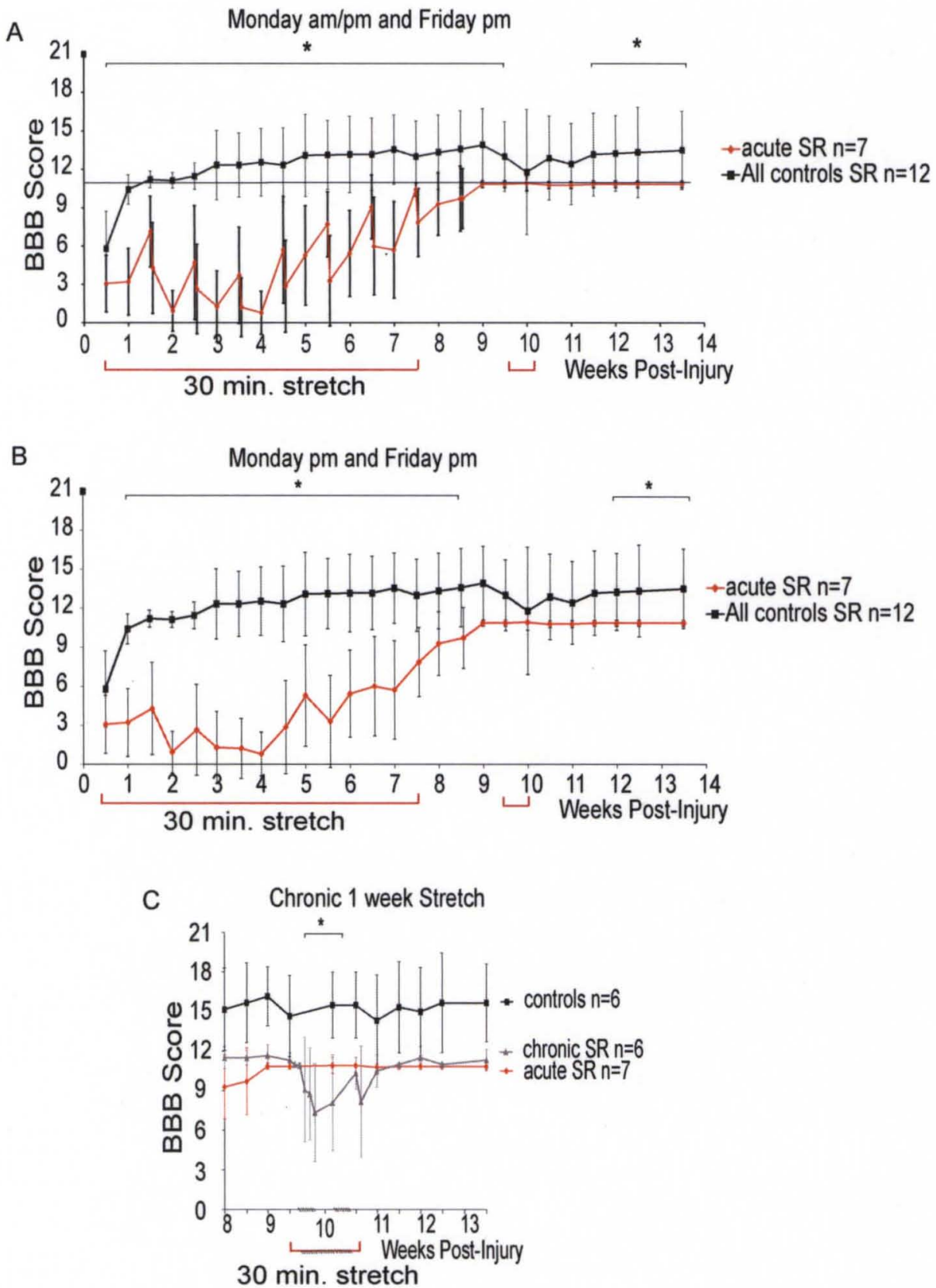
Continued BBB Scores and Handlers.

D, SR group individual animal BBB scores are presented for each week (Monday am, Monday $\mathrm{pm}$ and Friday $\mathrm{pm}$ ). The individual scores account for the large standard deviations found in the 8 weeks of daily stretch graph (Figure 3A). E, The difference in BBB scores (Monday amMonday $\mathrm{pm}$ ) plotted for each handler reveals no relationship between any handler and the loss of locomotor function in the rat he/she stretched on a given Monday.

C

BBB score 3 times weekly per Stretch animal
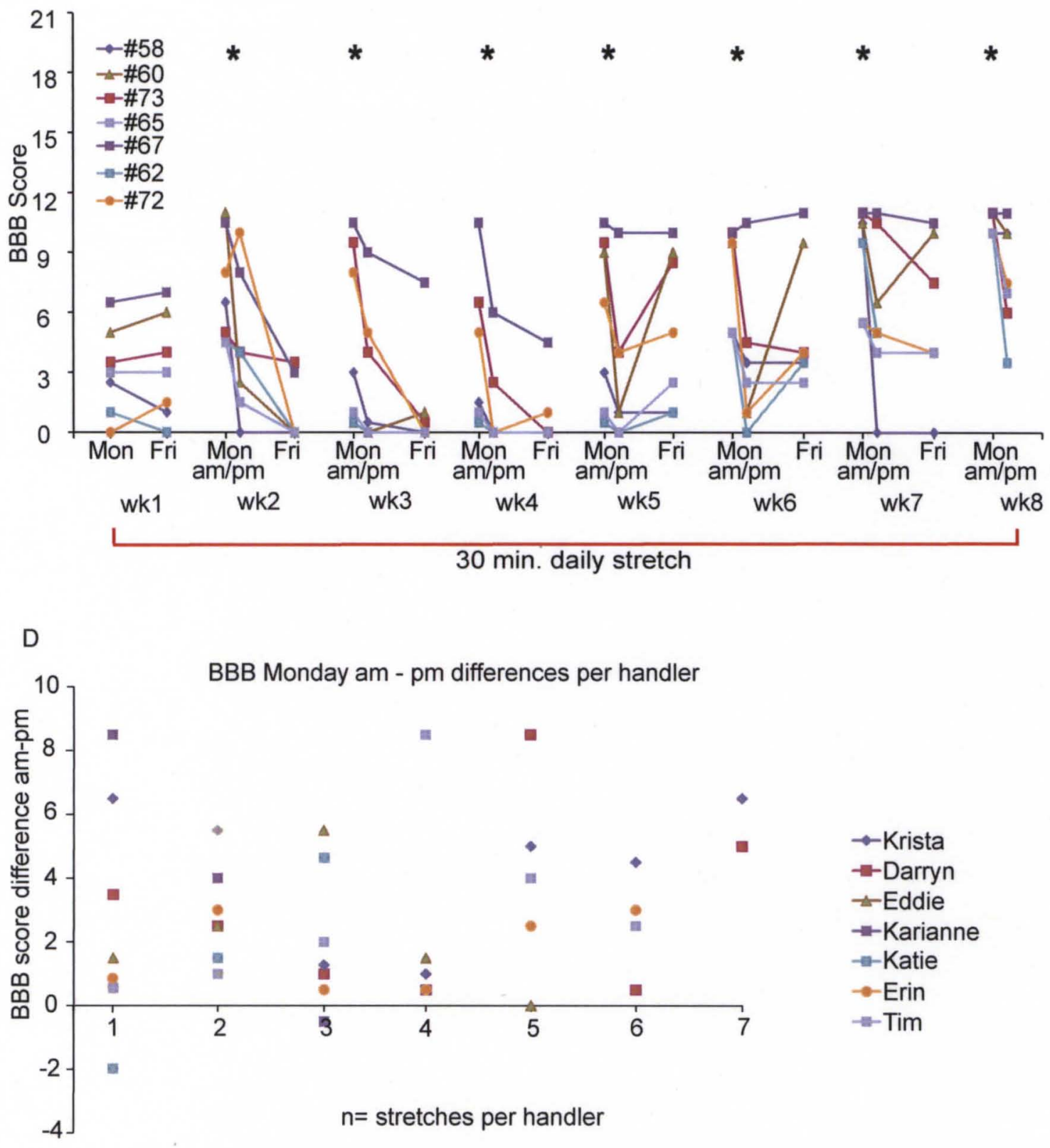
Figure 20. Swimming Assessments Daily Stretch.

A, During 8 weeks of daily SR protocol (red line under $x$-axis), the LSS scores for the SR group indicate a drop in swimming ability as compared to non-SR controls over weeks 2 and 4. The SR group experienced a gain of swimming function by week 4 and no significant differences in LSS score were evident thereafter. B, LSS scores are not significantly affected for the chronic SR group (grey trace) after 5 days of consecutive SR protocol measured at week 10.

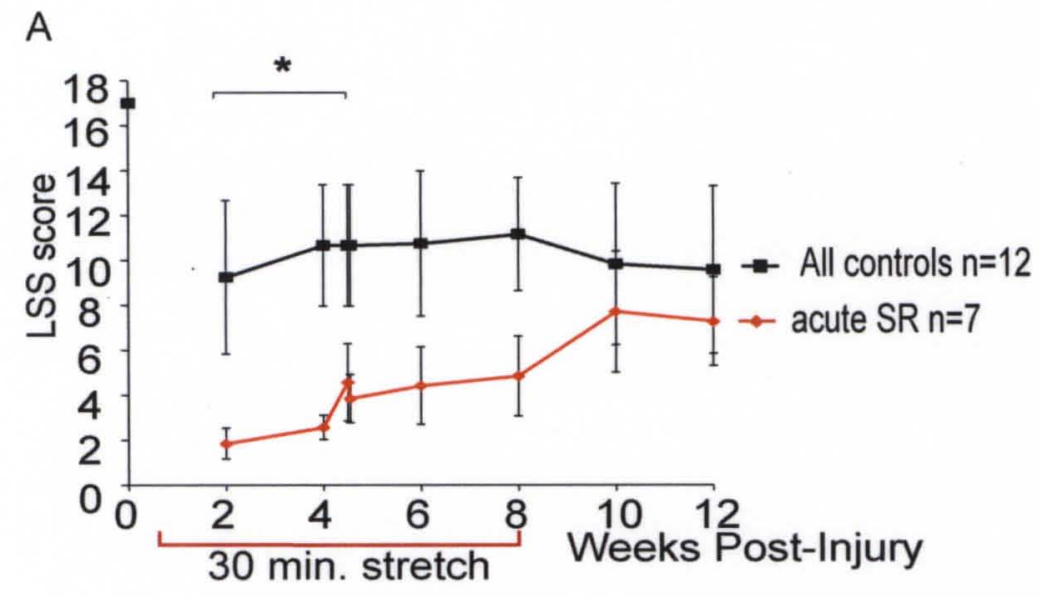

B

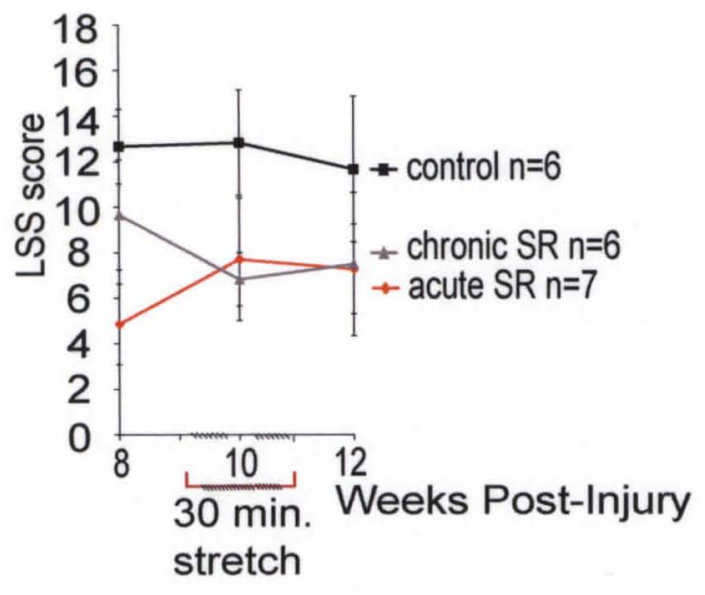


Figure 21. Passive range-of-motion (pROM) for Hindlimb Joints Daily Stretch.

A-C, Hip, knee, and ankle pROM was assessed using a manual goniometer. $A$, There is a significant loss in pROM for extension of the hip over time (within the first 4 weeks) as compared to baseline for both $S R$ and non-SR groups $(p \leq .05)$. pROM is recovered in the hip between weeks 4-6, there were no significant differences between groups at any week. B, The SR group experienced significant loss of pROM for extension of the knee as compared to the non-SR group beginning at week 4 that persisted through the duration of the study $(p \leq .05)$. No loss of flexion pROM in the hip or knee was observed. C, Flexion pROM in the ankle was affected, but no significant differences were evident.

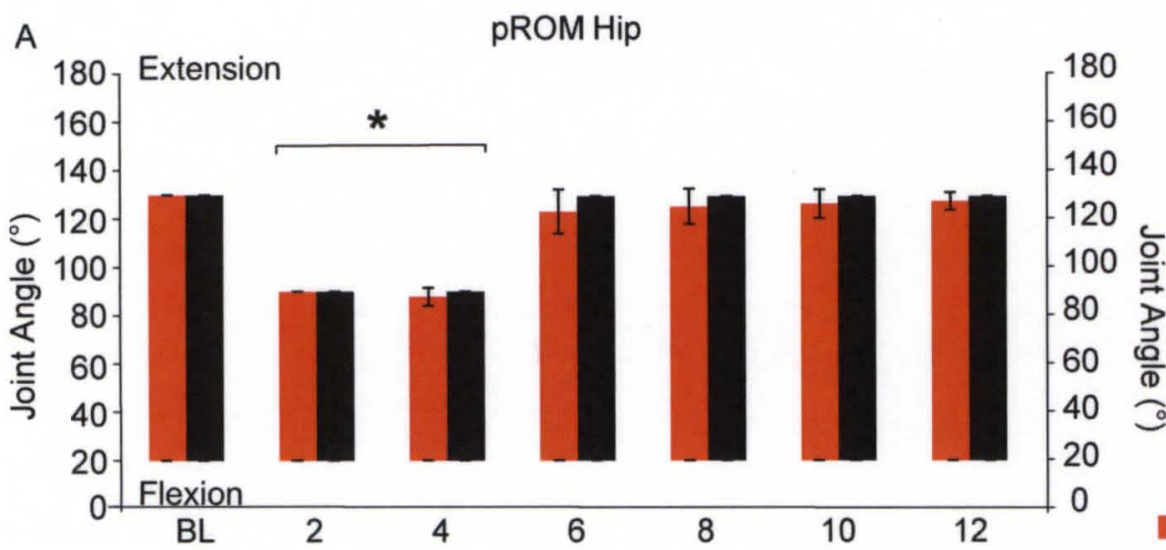

SR

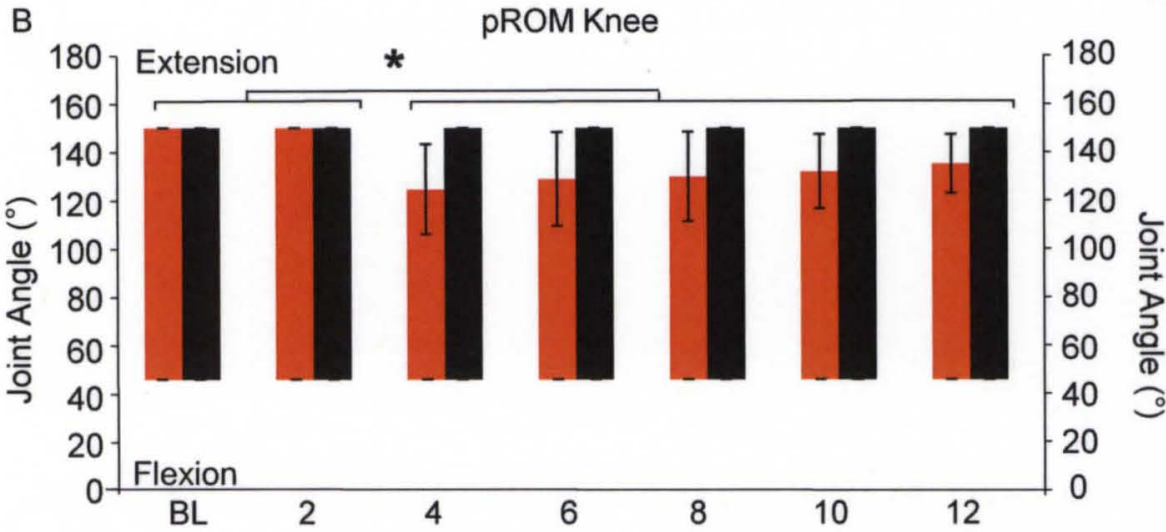

non-SR

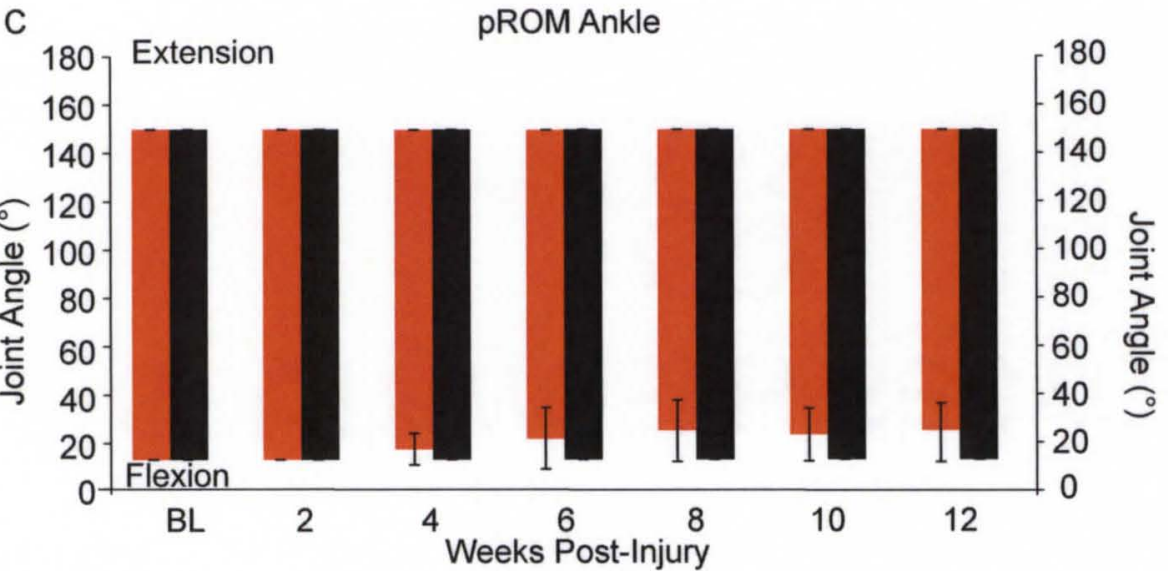


A
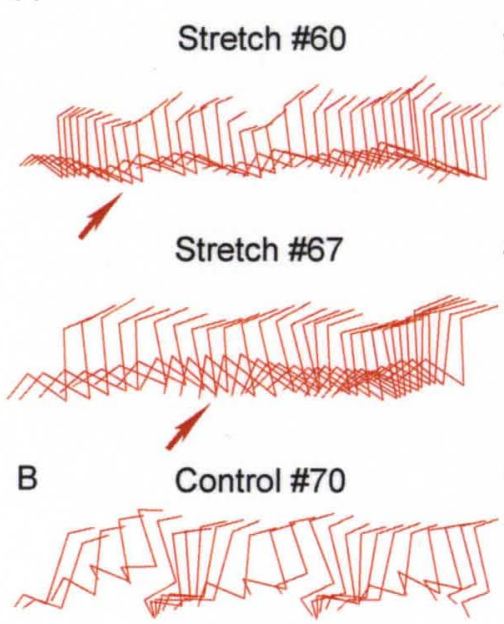

Control \#70

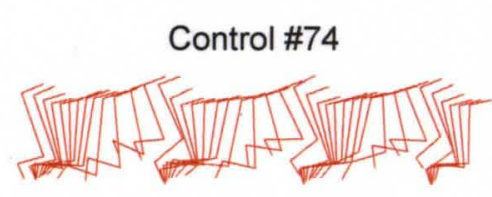

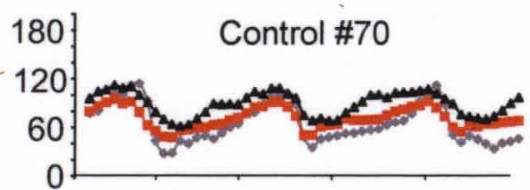

Week 3
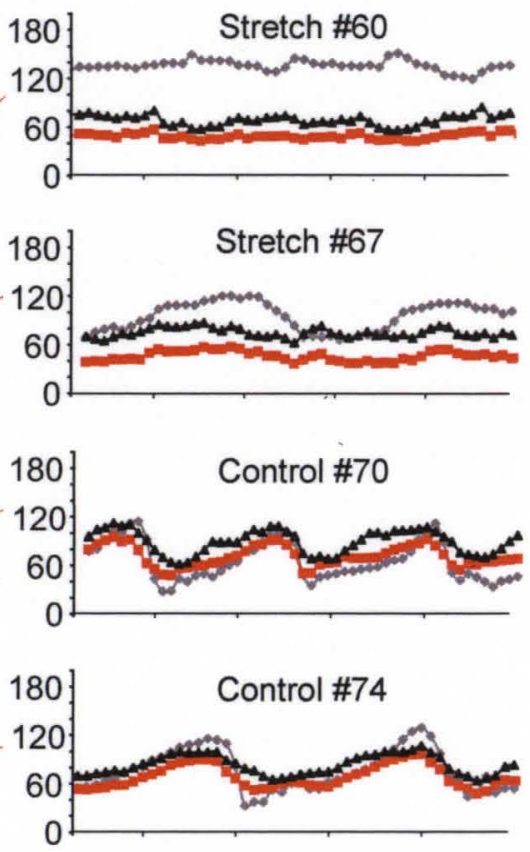

C
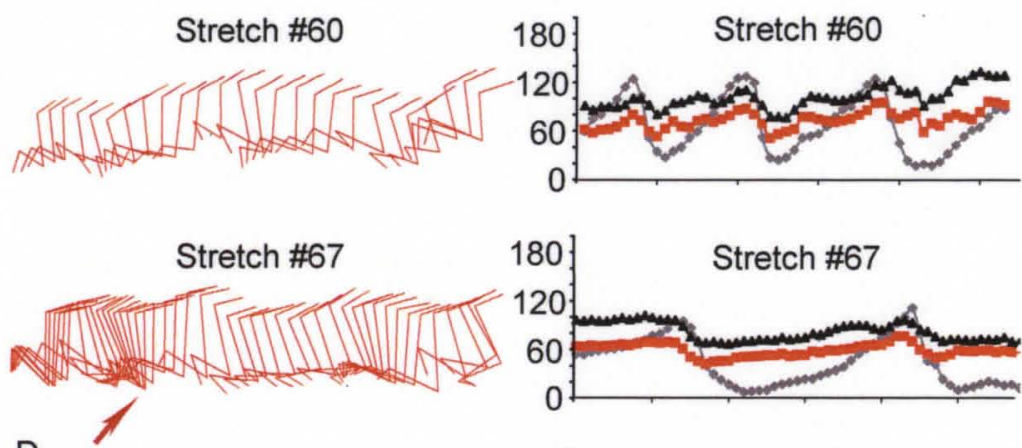

D
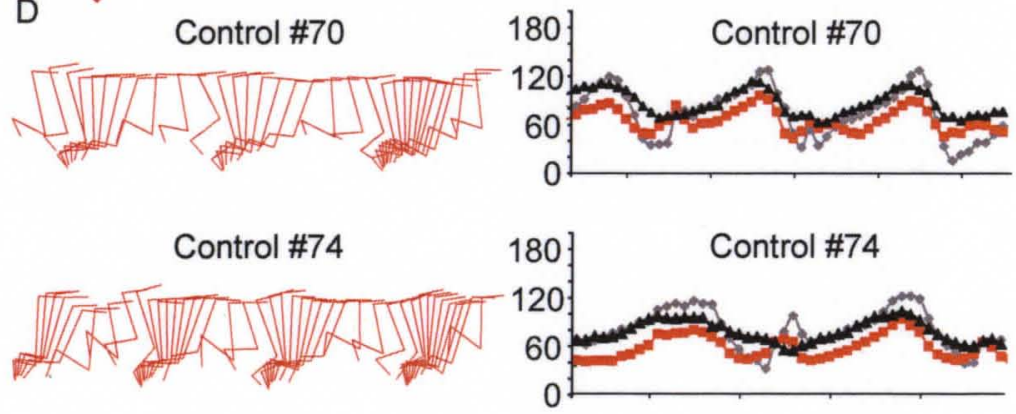

- Hip ( 70-115 $)$

- Knee ( 45-90 $)$

- Ankle ( 40-100 $)$

Figure 22. Intralimb Coordination Overground Stepping Daily Stretch.

A- D, Shown are representative 3D kinematic analysis during overground stepping. Right side excursion plots and stick figures were compared at 3 and 13 weeks, SR (A, C) and non-SR controls (B, D). A, At week 3 , the SR animals \#60 \& \#67 dragged their hindlimbs (knee down arrow) with little excursion in any joint. B, Non-SR animals were already capable of weight supported stepping at 3 weeks with cyclic excursion of all three hindlimb joints. C, By 13 weeks, \#60 SR animal had recovered swing and stance during stepping while \#67 still had knee down. The hip joint showed the greatest overall excursion with less recovery in the knee and ankle for both SR animals. D, Consistent with BBB scoring, the non-SR animals presented here did not show dramatic improvements in joint excursion at week 13. 
Figure 23. Pattern Formation and Plantar Stepping Shallow Water Walking Daily Stretch. $\mathrm{A}-\mathrm{C}$. CPI, PSI and RI measures for SR animals at week 3 indicating that they were dragging their hindlimbs, with no swing or stance phases due to lack of weight support. A and C. By 13 weeks, the CPI and RI indices of gait had recovered for SR animals. C. However, the RI shows that significant deficits still exist if the correct pattern is dependent upon plantar steps only. B. Importantly, the ratio of plantar stepping by the hindlimbs (compared to the forelimbs) was delayed but recovered by 13 weeks, indicating that the SR animals were able to weight support and achieved proper plantar paw placement in a few weeks without the daily stretch protocol.

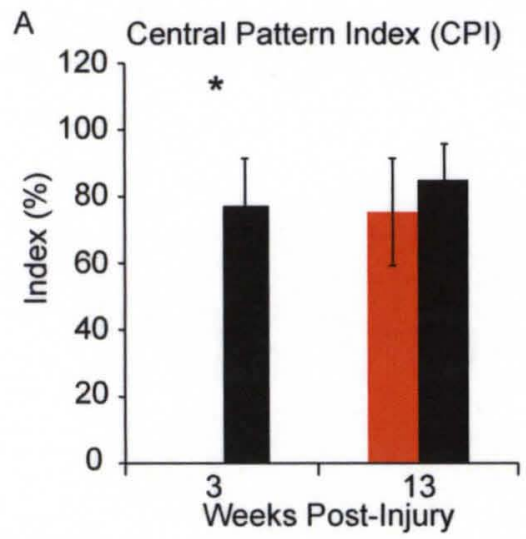

- acute SR

-All non-acute SR
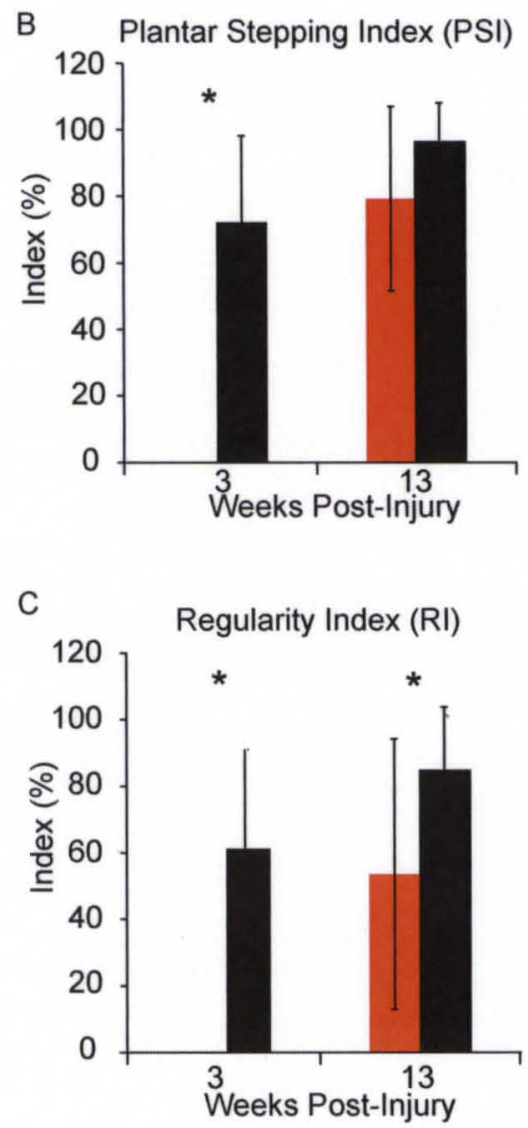
Figure 24. Immediate Stretch Responses (ISRs) Daily Stretch.

The ISR scores were compared between groups and over weeks. Stretch positions that involved flexion and extension of muscles controlling the ankle, knee and hip joints (A and $B$ ) have significantly higher ISR scores as compared to the hip adduction and abduction stretches (C). A and B. The ISR scores for ankle and knee stretches were significantly lower for the first Monday (week 0.5) as compared to Monday scores thereafter. C. No significant differences exist over weeks or between groups for ISR scores for hip abductors and adductors stretches.

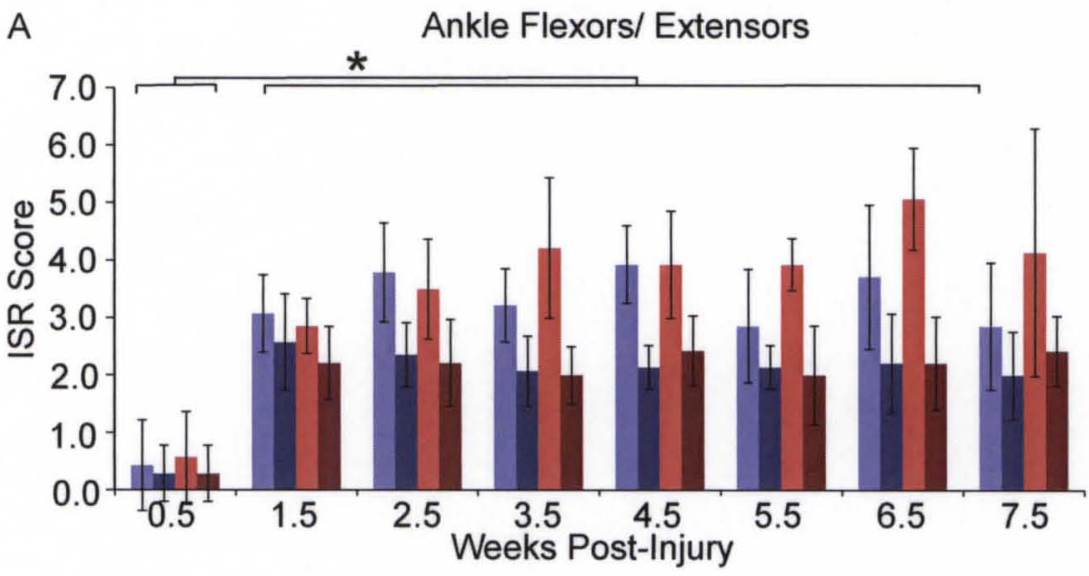

ankle flexors ipsi - ankle flexors contr - ankle extensors ipsi

- ankle extensors contr

B Knee Flexors/ Extensors

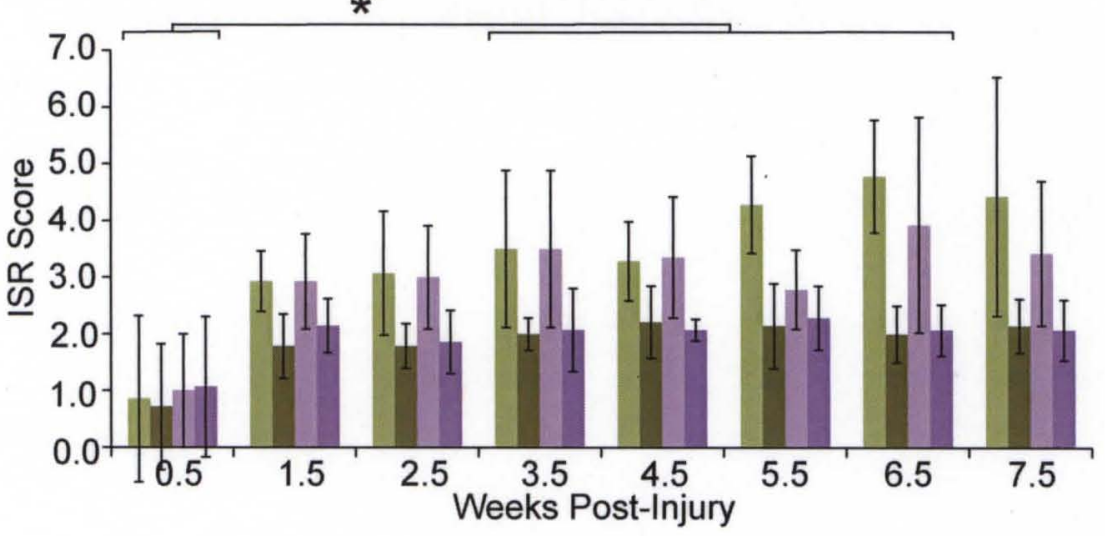

knee flexors ipsi

knee flexors contr

- knee extensors ipsi

knee extensors contr

C

Hip Adductors/ Abductors

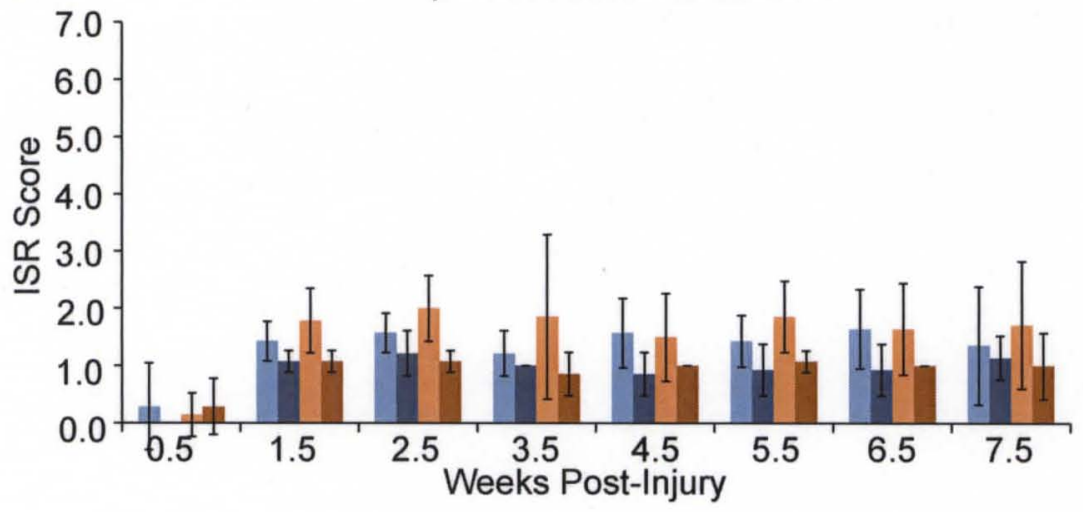

abductors ipsi

- abductors contr

adductors ipsi

adductors contr 
Table 3. Immediate Stretch Responses and BBB Score Correlations.

Pearson's correlation analysis was used to analyze all ipsilateral and contralateral ISR scores with afternoon BBB scores; significant correlations $r^{2} \leq .6$ are listed. 5 out of 6 significant correlations were from ISR scores on the contralateral limb, and 4 out of 6 have positive correlations. Table and A. ISRs are negatively correlated with pm BBB scores at Monday week 1.5 , but positively correlated at later Monday weeks 4.5 and 5.5 (circled time points). Other weeks showed no significant correlations. B and C. Scatter plots of representative correlations indicate that subsequent BBB scores may not be related to a particular hindlimb muscle group stretch; rather, in weeks 5-8 higher ISRs are associated with higher BBB scores.

Correlations: Immediate Stretch Response scores with Afternoon BBB scores (pm).

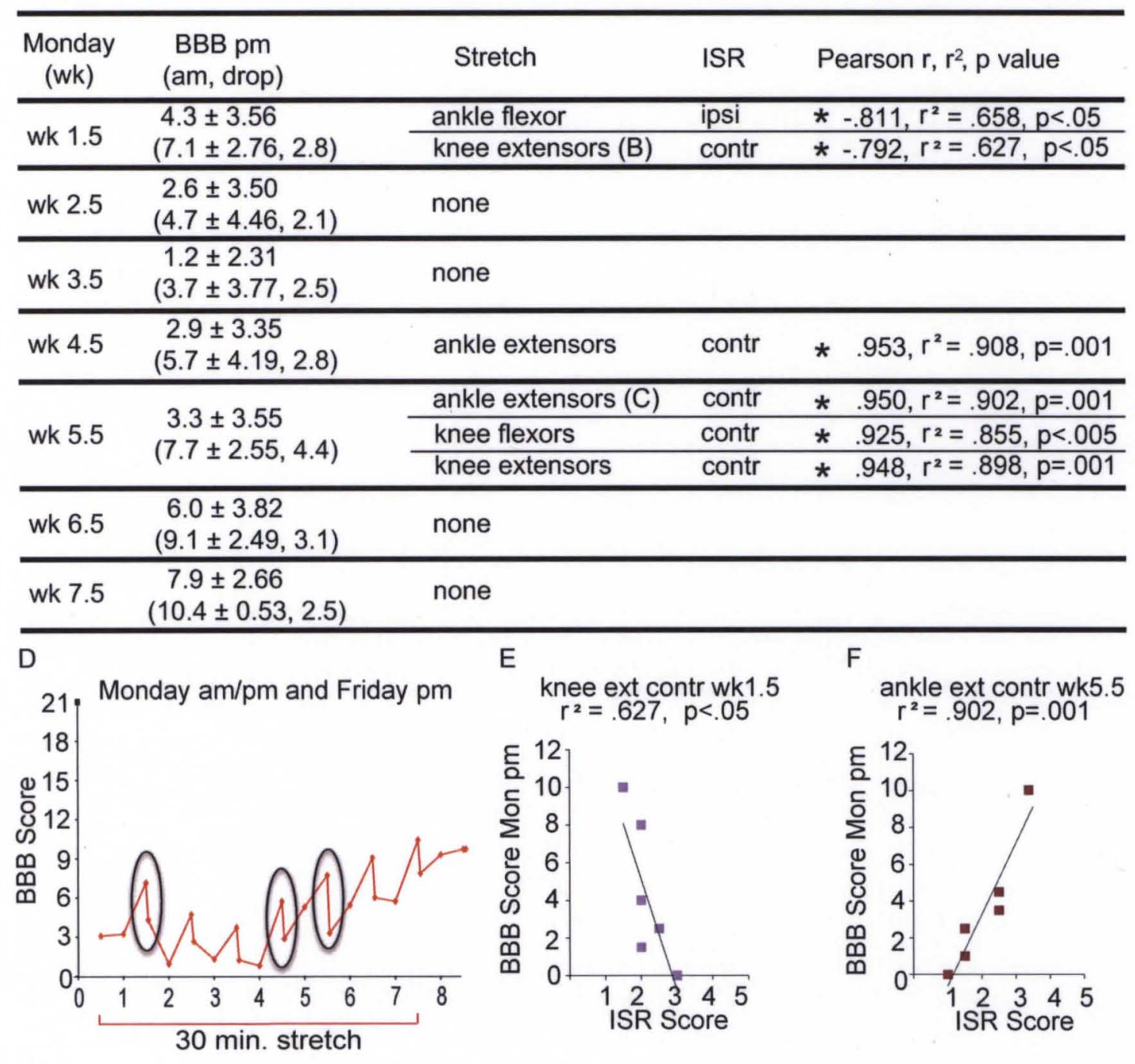


Figure 25. Magnetically Evoked Muscle Responses Daily Stretch.

A-C. Bilateral EMG recordings from LG were assessed after BBB scoring on Monday afternoon at weeks $3.5,6.5$ and 9.5. B. Week 6.5 showed significantly lower amplitude for SR group as compared to non-SR controls. D. There was a positive correlation for evoked response amplitude and BBB scores at week 6.5

A

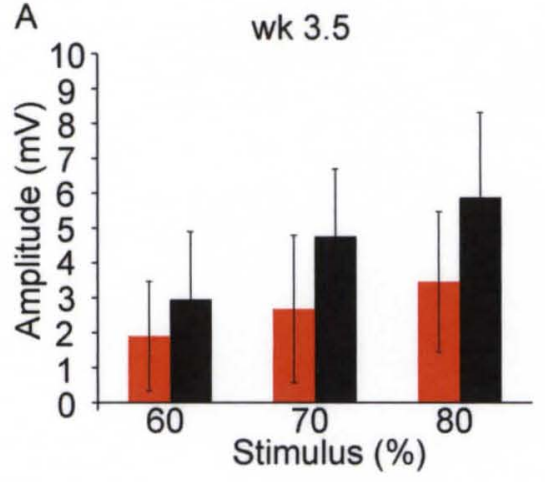

C

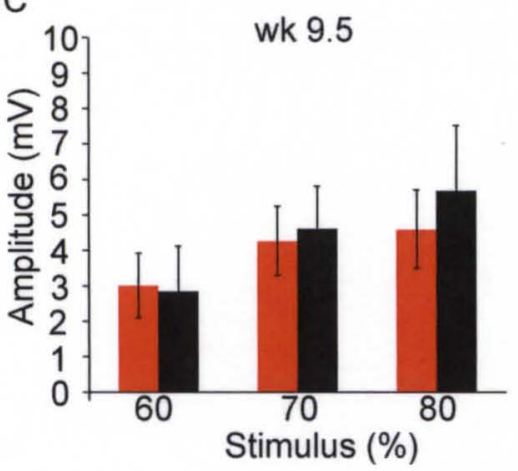

B

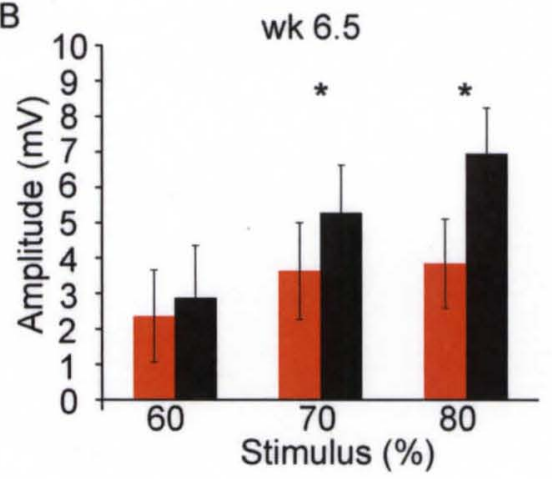

D

BBB $\times$ Amp wk6.5 Stretch

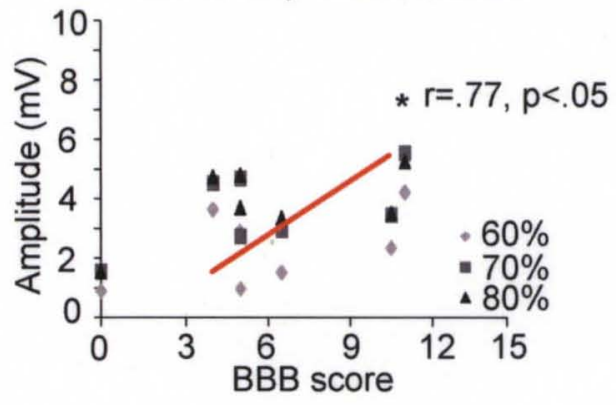


Body Weight (grams).

\begin{tabular}{lccccccc}
\hline Group & Baseline & wk 2 & wk 4 & wk 6 & wk 8 & wk 10 & wk 12 \\
\hline Stretch & $202.7 \pm 6.58$ & $204.9 \pm 10.04$ & $209.6 \pm 8.26$ & $218.0 \pm 9.63$ & $220.4 \pm 7.37$ & $232.9 \pm 5.98$ & $239.6 \pm 4.79$ \\
\hline controls $n=12$ & $197.9 \pm 4.45$ & $215.7 \pm 7.64$ & $221.1 \pm 9.09$ & $229.4 \pm 9.01$ & $235.7 \pm 10.67$ & $238.1 \pm 10.65$ & $245.8 \pm 9.51$ \\
\hline
\end{tabular}

Hindlimb Muscle Weight Significant Differences (grams).

\begin{tabular}{lcc}
\hline Group & ankle flexors (TA) & ankle extensors $(T S)$ \\
\hline Stretch & $\star 0.4487 \pm 0.0377$ & $\star 1.6160 \pm 0.1535$ \\
\hline controls $n=12$ & $0.5460 \pm 0.0660$ & $1.9176 \pm 0.1941$ \\
\hline
\end{tabular}

Spared White Matter at Injury Epicenter $\left(\mathrm{mm}^{2}\right)$.

\begin{tabular}{lc}
\hline Group & White Matter CSA \\
\hline Stretch & $15.9 \pm 5.38$ \\
\hline controls $n=12$ & $21.5 \pm 9.11$ \\
\hline
\end{tabular}

Table 4. Muscle Weight and Histology Daily Stretch.

Ankle extensors (TS) and flexors (TA) were significantly lighter for the SR group, however overall body weight was not different. Spared white matter at the epicenter was not significantly different between groups. 


\section{CHAPTER V}

\section{DISCUSSION}

Is In-Cage Training Real?

The wheelchair studies hinge on the hypothesis: Rats with contusion injuries maximally train themselves to walk, auto-train, early after low thoracic contusion $\mathrm{SCl}$ via in-cage activity. The "spontaneous" course of recovery, we believe, represents in-cage training. The WC studies described herein support this hypothesis by providing a negative control for in-cage activity of the hindlimbs. Possible afferent input while animals are in-cage training within days of injury include: dorsal paw cutaneous input from dragging the hindlimbs, rolling from side to side promotes passive range-of-motion of the joints, dragging the body forward may produce extension of the hip joint triggering reflexive flexion, sweeping using all three joints, and the animal may push backward onto the plantar paw surface and trigger weight-supported "pop up" stance and extensor loading. These actions are described by, and were the basis of, the BBB Open Field Scoring system for recovery of locomotor function after SCl in rats.

However, patients receive no such afferent input within days of $\mathrm{SCl}$.

Furthermore, the later stages of weight-supported plantar or dorsal stepping, followed by consistent stepping and then coordination are paralleled in step training for patients, but once again the timing of these actions are far beyond the first several days in most cases. In the case of laboratory animals, proper cues 
supplied to the nervous system to regain locomotion, coupled with volitional behaviors natural for the animal within weeks post-injury, may represent optimal activity-based functional locomotor recovery.

We have adapted our kinematic analysis system for overnight activity monitoring in which we track the distance traveled of individual rats during overnight activity. These quantitative systems are available commercially, however, since we use paired housing to promote in-cage activity and social interaction we had to develop a system capable of tracking multiple animals within the same cage. Our novel use of an activity monitoring system lies in our application of distance traveled (per rat) as an indication of the amount of afferent input to the hindlimbs (cutaneous stimulation/sweeps/cycles/steps) related to locomotor recovery. The expected results are that the distance travelled will increase as locomotor recovery improves as measured by the profile of BBB scores, and that hindlimb muscle activation and EMG reorganization will precede the emergence of locomotion. Edgerton and colleagues have previously characterized the EMG patterns of activation in hindlimb muscles of normal rats during daily in-cage activity, locomotion and posture, in singly housed female Sprague-Dawley rats (Hodgson et al., 2005). Their goal was to relate hindlimb muscle fiber type composition to how often a muscle was activated during everyday behaviors. In addition to no relationship found between fiber type and activation patterns, the authors were surprised to find very little duration of activity in TA, up to 3 hours per 24 hour period, while MG, VL and soleus were active for up to $\sim 9,12$ and 15.5 hours respectively. In a subsequent study the 
authors used complete spinal isolation (SI) technique of double transections, midthoracic and high sacral segments, along with rhizotomy of adjacent dorsal roots to characterize activation of functionally isolated muscles (Roy et al., 2007). The authors found that SI eliminates at least $98 \%$ of extensors and only $92 \%$ of TA activation. Interestingly, the TA is one of the least active muscles of the hindlimb during normal daily activity, however it is one of the most spontaneously active when functionally isolated. So between these two studies we have an indication of the baseline amounts of activation in normal and functionally isolated, but healthy, hindlimb muscles, so data from our proposed studies on hindlimb EMG activity of incomplete SCI during in-cage activity should fall between activation levels in these studies. However our focus is to study functional locomotor recovery as measured by our standard outcome measures of the BBB scale and hindlimb kinematics, rather than muscle fiber type.

Stress and Restraint

The point is well taken that we should consider stress in our models since both wheelchairs and stretching involve restraint. The main reason we are not often questioned about stress from colleagues that do activity-based rehabilitation and review our publications is that stress on a systems level of physiology and behaviorally is intrinsic and clinically relevant. Since our lab focuses on behavioral neuroscience we may consider the degree to which stress affects behaviors in our model. Animals raised in social environment are more resilient to stress (Konkle et al., 2010), and when stress is repeatedly applied young adult rats are no different from controls when measured in open field exploratory behaviors (Shoji and Mizoguchi, 2010). Our model includes repeated 
stress and social interaction, both factors that mitigate negative effects of stress. Furthermore, our model is difficult to compare to other immobilization and restraint studies because they measure exploratory motor behaviors once the restriction is terminated whereas we are interested in the amount of motor activity and stress while the animals are in the wheelchairs.

Our model is not vastly different from activity-based rehabilitation strategies in terms of how mush restraint is necessary. BWST step training in also involves strapping the animal into a tight body vest with the forelimbs/hindlimbs free for movement. Many models require the animals to step bipedally, not a natural posture for quadrupedal animals, and in higher injuries (T3 or cervical) induces significant stress of autonomic dysrelfexia and interrupts breathing, heart rate, and blood pressure. Furthermore, the alternative models such as tail suspension and casting, we would argue, are not any less stressful for the animals. Our model differs from cast immobilization models because it allows animals to move about despite the various degrees of hindlimb immobilization. Casting has not been well studied in $\mathrm{SCl}$ models, and the one study that has used it (Ye et al., 2012) only casted for 21 days, far less than that of chronic SCl patients would experience. In other words, this model may be even more stressful and uncomfortable than our model that includes extra measures for the comfort, pressure relief and circulation.

A proposed method to control for stress in our wheelchair model is to place all the animals in wheelchairs at least temporarily daily. Therefore all animals would experience the restraint stress but non-WC animals would be 
removed from wheelchairs within $\sim 30$ minutes so that cumulative in-cage activity would not be disrupted. Similarly in the stretching studies we could restrain the non-Stretch control animals and manipulate their forelimbs for $\mathbf{3 0}$ minutes to parallel that of the hindlimb stretch 30 -minute protocol.

Clinically Relevant SCI

We believe that the use of transection models in extrapolation for the promise of recovery due to activity-based rehabilitation is a misstep by our field, and that incomplete injuries are more relevant clinically since most patients do not experience a complete anatomical transection (Bunge et al., 1993; Kakulas, 1999; Norenberg et al., 2004). However, we must recognize the remaining differences between experimental $\mathrm{SCl}$ in animal models and patients: 1) upper thoracic and cervical level injuries represent the majority of SCls clinically while animal studies of locomotor training have consistently used low thoracic lesions and 2) SCI patients have predominantly ventral lesions while animal models enter from the posterior aspect and apply a dorsal lesion (Norenberg et al., 2004). Lesions of the VLF are devastating to locomotor function, but even very little sparing in humans $(10 \%$, and only $5 \%$ in rats) is sufficient for locomotor output (Majczynski and Slawinska, 2007). A rim of spared white matter remains intact in even the most severe ventral SCls in patients yet spared function is poor (Bunge et al., 1993, Kakulas 1999). Prognosis is also poor for patients with anterior cord syndrome, a lesion primarily affecting the VLF; while on the other hand, functional recovery from posterior cord syndrome is often substantial (McKinley et al., 2007). Therefore, clinical data supports increased use of ventral rather than dorsal lesions in experimental animal models. A primary reason 
experimental $\mathrm{SCl}$ models do not use higher level and/or ventral lesions is that they are exponentially more technically difficult, are highly invasive requiring several hours of surgery and complex post-operative care, and more expensive. If we were to include ventral lesions in rats we would have to be sure to obliterate all of the VLF, a graded laceration study would be neither feasible nor useful since even the slightest bit of sparing would result in no loss of locomotion. A reasonable compromise to make our models more clinically relevant is to do ventral lesions (much like Gorska and colleagues' standout studies on overground recovery of locomotion in cats two decades ago) at a low thoracic level, thereby increasing clinical relevance but keeping the costs of surgeries low and chance of animal survival high. Inter-enlargement Communication Although we did not consider it during the development of our studies, we have essentially created a model of forelimb training secondary to limiting/delaying hindlimb locomotor recovery in a wheelchair. We view wheelchair immobilization as a negative control for hindlimb locomotor activity that contributes to functional recovery after $\mathrm{SCl}$. WC animals also undergo a form of alternating cyclic forelimb training because they are completely dependent upon the forelimbs as they maneuver about in their cages. Indeed the purpose of wheelchairs in patients is to increase mobility; rather than being bed bound a patient can move throughout his/her environment in a wheelchair, and is mostly dependent on spared function in the upper limbs. Rats maneuvering in wheelchairs may have experienced less resistance (more mobility in their wheelchairs) than dragging their hindlimbs, which may have resulted in more 
forelimb steps than achieved by non-WC control animals dragging their hindlimbs. Regardless, the alternating forelimb steps performed by quadrupedal animals recovering from paraplegia is not like that of wheelchair bound patients who do not alternate their arms when propelling manual wheelchairs. Both the amount and pattern of movement of the upper extremities/forelimbs affects plasticity and propriospinal inter-enlargement connections. Our University of Louisville colleagues at the Frazier Rehab Institute stress the importance of rhythmic arm swing during BWST step training (Behrman and Harkema, 2000), (Berhman presentation KSCIRC/Frazier Rehab Institute, Spring 2012), and the Zehr laboratory (University of Victoria) has led our field in studying of the effects of rhythmic activation of whole arm swing on lumbar excitability. Zehr and colleagues have shown rhythmic arm movement itself and sensory afferent input, via $\mathrm{H}$-reflex and cutaneous stimulation, applied to the arm during swing have a positive effect on excitability in lower limbs during stepping (Zehr and Chua, 2000; Ferris et al., 2001; Zehr, 2005). Of particular relevance is the study by Klimstra et al. showing that the specific excursions of the arm joints may differ based on the locomotor task (swing during stance or stepping, and arm cycling), but the rhythmic alternating pattern was the most important component of afferent input for inducing excitability in the lower limbs (2009). In essence, our locomotor output is the result of upper and lower limb CPGs wired together such that we have all "four limbs walking" (Dr. David Bashor personal communication). The kinematics of the arms during wheelchair push rim propulsion has been studied extensively because arm excursions have differential effects on shoulder 
strain, pain, fatigue and aerobic benefits (van der Woude et al., 2001). Similarly, upper limb training in the form of hand cycling/arm crank training is well established in the $\mathrm{SCl}$ neurorehabilitation field because of its ability to bring about increases in cardiopulmonary function (Jack et al., 2010; Hostettler et al., 2012) as a single intervention, and when paired with functional electrical stimulation (FES) (Coupaud et al., 2008). The more recent arm crank studies use synchronous (in phase) rather than an asynchronous (alternating, reciprocal, out of phase) mode of arm crank training because it is most relevant to the common wheelchair design of push rim propulsion. Studies comparing the two modes in uninjured normal subjects have mixed results for cardiovascular and aerobic outcome measures likely due to the extent in which trunk muscles were recruited and the specific test conditions (Dallmeijer et al., 2004; van der Woude et al., 2008). At least one study comparing the two modes in SCl patients (that were unable to recruit trunk musculature) reported a preference for the asynchronous mode, but the effects on cardiovascular outcomes were not robust for either mode (Mossberg et al., 1999). Interestingly, a few decades ago Engel and Hildebrandt developed an asynchronous push/pull lever wheelchair (1974), van der Woude et al. expanded this design (1993), and others showed that asynchronous push rim propulsion with high gear ratio required less effort (Glaser et al., 1980b; Glaser et al., 1980a). Currently, hand cycle equipment can be added to traditional push rim designs, but these forms of adapted wheelchairs are not available to the majority of manual wheelchair users (Requejo et al., 2008). However, arm crank/cycle ergometers could be easily integrated into 
more rehabilitation settings and adapted as a training tool to ramp up central excitation for lower limb activity during/in conjunction with step training. As of October 2012, Dr. Zehr was not aware of any data, unpublished or published, in which alternating arm swing, forelimb stepping, or arm crank/cycling training after $\mathrm{SCI}$ has been used to study functional locomotor recovery outcome measures specifically in the lower limbs (Zehr, E. P., personal communication). These effects are likely due to activation of long propriospinal interneurons responsible for inter-enlargement connections, and the likely anatomical target of synaptic plasticity (Juvin et al., 2005; Flynn et al., 2011). Though forelimb stepping in a quadruped may not have the same qualitative afferent components as arm swing in patients, two factors may be of great importance if we consider that WC animals maintained capacity for locomotion early after injury via forelimb activation: 1) as the animals pulled/maneuvered themselves forward the movements were volitional, and 2) the movements were alternating. It is safe to assume that the majority of patients have great amounts of upper limb activation during wheelchair propulsion, however the lack of patterned left-right alternation during synchronous push rim propulsion may be suboptimal coupling of cervical and lumbar enlargements coordinating locomotion.

\section{Extrapolation}

Our studies suggest that the CNS is not being tapped to its fullest capacity with current neurorehabilitation training paradigms. There exists abundant computational power, through redundancy and convergence, hard wired within the CNS that the connection/ability can just "pop out" if the system is supplied with enough excitation, an optimal combination of afferent input cues, and a 
memory/frame of reference for what it needs to achieve behaviorally. We have not yet struck the optimal combination of activity in all the distributed CNS centers contributing to locomotion. In other words we need afferent cues in many more modalities than just those of the lower limbs. If the entire CNS system for locomotion is activated during locomotor rehabilitation: the spared spinal cord tissue, including upper and lower enlargement CPGs, ascending and descending propriospinal interneurons, brainstem centers and cerebellum, visual and vestibular systems and cortical representations, then the CNS may better activate the circuit that produces the behavior of locomotion. A rough locomotor pattern may be prompted by afferent input during locomotor training, however movement is often compensatory (Harkema et al., 2012a) and the training focuses only on that segment of the body. I believe that in the majority of anatomically incomplete spinal cord injuries the central nervous system can rewire (synaptically) and reinforce (with practice) the necessary activations for concerted volitional locomotion without needing to regenerate axons through the lesion at all.

The involvement of afferent cues of the visual system (in addition to a mirror during step training), balance, and axial musculature may not be fully understood. The issue of balance is identified in the field of step training in both animal models and patients. Our studies suggest that potentially noxious afferent input in the form of hindlimb immobilization and stretch negatively effected hindlimb movement, but also balance and stability that are closely tied to the ability to support body weight. Balance is usually assisted in animal models and 
patients stepping on a treadmill, and often during overground stepping even when the patient is relatively high functioning. Aiding in balance is necessary during step training to control for injury and risk of falls, however training for stability and balance by itself usually involves stand straining while maintaining center of balance after a perturbation. Locomotor training studies in which the two girdles of the axial musculature (shoulders and hips) are specifically targeted with a reciprocally coupled and rhythmic pattern are needed. For example, a dynamic stability rehabilitation approach that focuses on rhythmic activity in axial musculature would be ideal to retrain trunk muscles (would have to include body weight support). It is in this manner that BWS crawling as a form of locomotor training might be useful. We are often questioned as to whether we think patients should be crawling in order to avoid being immobilized early after injury. This is a plausible suggestion but may not be feasible when so many patients suffer multiple traumas that take several weeks or months of immobility in order to heal. Yet, crawling has several other factors of locomotion that we should consider for $\mathrm{SCl}$ patients who want to increase volitional mobility. During crawling a patient would have added stability with a lower center of gravity and a reduced risk of injury if a fall occurs. Therefore trainers may be able to challenge the amount of weight support more frequently and perhaps shift priority from high intensity/repetition of movements towards incremental smaller movements that are wholly volitional. The hips, shoulders, and axial musculature may be able to alternate and train specific relationships present during crawling and stepping. Crawling may incite a frame of reference, although only used early in 
development, that engages several locomotor control systems. Volitional crawling and maintenance of balance on all fours may be an incremental phase of locomotor recovery and may be "purer" than completely aid dependent ambulation based on compensation. Even if crawling on all fours is not be feasible for all patients within the first weeks post-injury, some other training strategy that activates shoulders, hips, and trunk musculature in an alternating rhythmic pattern may be of some value.

Optimal Training Strategy

An optimal rehabilitation program would incorporate the daily use of a common wheelchair fitted with an asynchronous arm crank to maneuver at all times. The patient would never have to perform synchronous push rim mechanics required by virtually all commercially available wheelchairs. Activitybased rehabilitation training in a clinical setting would begin as early as two weeks post-injury and high intensity muscle stretch would be avoided completely until the patient developed debilitating spasticity or contracture of no greater than $1 / 3$ of total joint ROM. At any point in which stretch is necessary it should not exceed 30 seconds and $60 \mathrm{Nm}$ of intensity since Harvey et al. (2003) reported that is the upper limit of torque tolerated by sensate neurologically normal individuals. Activity-based rehabilitation starting at two weeks would incorporate two pairs of rehabilitation regimens that are alternated every other session throughout at least three months of daily training: 1) 30 minutes of high intensity asynchronous mode arm crank cycling (with trainer support if quadriplegic) followed by BWS overground crawling in which the lower limbs are moved by trained therapists as the patient crawls (rhythmically alternating pattern, 
reciprocal with upper limb "steps") and air stepping training (triggered by providing $100 \%$ body weight support, hip extension applied by therapist, and upper limb asynchronous cycling), 2) 30 minutes of high intensity asynchronous mode arm crank cycling followed by BWST step training as already established at Frazier Rehab Institute by Dr. Harkema and Dr. Behrman. Patients with improved crawling, air stepping, volitional movements, or greater postural stability may have more easily excitable spared spinal cord structures below the level of injury resulting in greater outcomes due to BWST step training. The goal is not to train people to crawl or air step perfectly. The goal is to ramp up the limiting factors to successful bipedal weight-bearing locomotion (lack of postural stability, decreased central excitation, loss of intrinsic CPG function evident early post-injury because it is not maintained) so that already established BWST step training methods may be enhanced.

Conclusion

Our studies have helped open the consideration that common practices and treatments for $\mathrm{SCl}$ patients are detrimental to functional recovery. After $\mathrm{SCl}$ descending drive for locomotion is greatly diminished from supraspinal centers (MLR, SLR) because descending pathways (DC, DLF, and VLF) are interrupted, and the spinal structures controlling intrinsic locomotion, if spared, become exquisitely sensitive to their remaining inputs, the afferents. Therefore any and all afferent input is vitally important for synaptic transmission and reorganization. The complex profile of functional recovery we have uncovered does not support current practices and standard of care for neurorehabilitation since our interventions during the first few weeks negatively shaped potential for recovery 
in later weeks. Current neurorehabilitation efforts are missing a critical time window of optimal plasticity. We attribute the afferent input of immobilization and stretch as maladaptive; so minimizing these factors while maximizing functional afferent activity applied early within weeks post-injury (possibly crawling, arm cycling, and/or step training) would support optimal functional recovery if moved to clinical application. 


\section{REFERENCES}

Abbadie C, Trafton J, Liu H, Mantyh PW, Basbaum Al (1997) Inflammation increases the distribution of dorsal horn neurons that internalize the neurokinin-1 receptor in response to noxious and non-noxious stimulation. J Neurosci 17:8049-8060.

Abelew TA, Miller MD, Cope TC, Nichols TR (2000) Local loss of proprioception results in disruption of interjoint coordination during locomotion in the cat. J Neurophysiol 84:2709-2714.

Adelson D, Lao L, Zhang G, Kim W, Marvizon JC (2009) Substance P release and neurokinin 1 receptor activation in the rat spinal cord increase with the firing frequency of $\mathrm{C}$-fibers. Neuroscience 161:538-553.

Agrawal SK, Fehlings MG (1996) Mechanisms of secondary injury to spinal cord axons in vitro: role of $\mathrm{Na}+, \mathrm{Na}(+)-\mathrm{K}(+)-A T P a s e$, the $\mathrm{Na}(+)-\mathrm{H}+$ exchanger, and the $\mathrm{Na}(+)-\mathrm{Ca}+$ exchanger. J Neurosci 16:545-552.

Alluin $\mathrm{O}$, Karimi-Abdolrezaee $\mathrm{S}$, Delivet-Mongrain $\mathrm{H}$, Leblond $\mathrm{H}$, Fehlings MG, Rossignol S (2011) Kinematic study of locomotor recovery after spinal cord clip compression injury in rats. J Neurotrauma 28:1963-1981.

Barbeau H, Rossignol S (1987) Recovery of locomotion after chronic spinalization in the adult cat. Brain Res 412:84-95.

Barbeau H, Ladouceur M, Norman KE, Pepin A, Leroux A (1999a) Walking after spinal cord injury: evaluation, treatment, and functional recovery. Arch Phys Med Rehabil 80:225-235.

Barbeau H, McCrea DA, O'Donovan MJ, Rossignol S, Grill WM, Lemay MA (1999b) Tapping into spinal circuits to restore motor function. Brain Res Brain Res Rev 30:27-51. 
Bareyre FM, Kerschensteiner M, Raineteau O, Mettenleiter TC, Weinmann O, Schwab ME (2004) The injured spinal cord spontaneously forms a new intraspinal circuit in adult rats. Nat Neurosci 7:269-277.

Barriere G, Leblond H, Provencher J, Rossignol S (2008) Prominent role of the spinal central pattern generator in the recovery of locomotion after partial spinal cord injuries. J Neurosci 28:3976-3987.

Basso DM, Beattie MS, Bresnahan JC (1995) A sensitive and reliable locomotor rating scale for open field testing in rats. $J$ Neurotrauma 12:1-21.

Basso DM, Beattie MS, Bresnahan JC (2002) Descending systems contributing to locomotor recovery after mild or moderate spinal cord injury in rats: experimental evidence and a review of literature. Restor Neurol Neurosci 20:189-218.

Basso M, Hansen CN (2011) Biological basis of exercise-based treatments: spinal cord injury. PM R 3:S73-77.

Battaglino RA, Lazzari AA, Garshick E, Morse LR (2012) Spinal Cord InjuryInduced Osteoporosis: Pathogenesis and Emerging Therapies. Curr Osteoporos Rep.

Battistuzzo CR, Callister RJ, Callister R, Galea MP (2012) A systematic review of exercise training to promote locomotor recovery in animal models of spinal cord injury. J Neurotrauma 29:1600-1613.

Baumbauer KM, Young EE, Hoy KC, Jr., Joynes RL (2007a) Intrathecal administration of neurokinin 1 and neurokinin 2 receptor antagonists undermines the savings effect in spinal rats seen in an instrumental learning paradigm. Behav Neurosci 121:186-199.

Baumbauer KM, Young EE, Hoy KC, Jr., Joynes RL (2007b) Neurokinin receptors modulate the impact of uncontrollable stimulation on adaptive spinal plasticity. Behav Neurosci 121:1082-1094.

Beare JE, Morehouse JR, DeVries WH, Enzmann GU, Burke DA, Magnuson DS, Whittemore SR (2009) Gait analysis in normal and spinal contused mice using the TreadScan system. J Neurotrauma 26:2045-2056. 
Behrman AL, Harkema SJ (2000) Locomotor training after human spinal cord injury: a series of case studies. Phys Ther 80:688-700.

Belanger M, Drew T, Provencher J, Rossignol S (1996) A comparison of treadmill locomotion in adult cats before and after spinal transection. $J$ Neurophysiol 76:471-491.

Ben M, Harvey LA (2010) Regular stretch does not increase muscle extensibility: a randomized controlled trial. Scand J Med Sci Sports 20:136-144.

Beres-Jones JA, Johnson TD, Harkema SJ (2003) Clonus after human spinal cord injury cannot be attributed solely to recurrent muscle-tendon stretch. Experimental Brain Research 149:222-236.

Berg HE, Larsson L, Tesch PA (1997) Lower limb skeletal muscle function after 6 wk of bed rest. J Appl Physiol 82:182-188.

Bigbee AJ, Crown ED, Ferguson AR, Roy RR, Tillakaratne NJ, Grau JW, Edgerton VR (2007) Two chronic motor training paradigms differentially influence acute instrumental learning in spinally transected rats. Behav Brain Res 180:95-101.

Bolton DA, Tse AD, Ballermann M, Misiaszek JE, Fouad K (2006) Task specific adaptations in rat locomotion: runway versus horizontal ladder. Behav Brain Res 168:272-279.

Boorman GI, Lee RG, Becker WJ, Windhorst UR (1996) Impaired "natural reciprocal inhibition" in patients with spasticity due to incomplete spinal cord injury. Electroencephalogr Clin Neurophysiol 101:84-92.

Bouyer LJG, Rossignol S (2003a) Contribution of Cutaneous Inputs From the Hindpaw to the Control of Locomotion. I. Intact Cats. J Neurophysiol 90:3625-3639.

Bouyer LJG, Rossignol S (2003b) Contribution of Cutaneous Inputs From the Hindpaw to the Control of Locomotion. II. Spinal Cats. J Neurophysiol $90: 3640-3653$. 
Bunge RP, Puckett WR, Becerra JL, Marcillo A, Quencer RM (1993) Observations on the pathology of human spinal cord injury. A review and classification of 22 new cases with details from a case of chronic cord compression with extensive focal demyelination. Adv Neurol 59:75-89.

Burke DA, Magnuson DS, Nunn CD, Fentress KG, Wilson ML, Shum-Siu AH, Moore MC, Turner LE, King WW, Onifer SM (2007) Use of environmentally enriched housing for rats with spinal cord injury: the need for standardization. J Am Assoc Lab Anim Sci 46:34-41.

Cai LL, Courtine G, Fong AJ, Burdick JW, Roy RR, Edgerton VR (2006a) Plasticity of functional connectivity in the adult spinal cord. Philos Trans $R$ Soc Lond B Biol Sci 361:1635-1646.

Cai LL, Fong AJ, Otoshi CK, Liang Y, Burdick JW, Roy RR, Edgerton VR (2006b) Implications of assist-as-needed robotic step training after a complete spinal cord injury on intrinsic strategies of motor learning. J Neurosci 26:10564-10568.

Canu MH, Garnier C (2009) A 3D analysis of fore- and hindlimb motion during overground and ladder walking: comparison of control and unloaded rats. Exp Neurol 218:98-108.

Carvalho KA, Cunha RC, Vialle EN, Osiecki R, Moreira GH, Simeoni RB, Francisco JC, Guarita-Souza LC, Oliveira L, Zocche L, Olandoski M (2008) Functional outcome of bone marrow stem cells (CD45(+)/CD34(-)) after cell therapy in acute spinal cord injury: in exercise training and in sedentary rats. Transplant Proc 40:847-849.

Castro MJ, Apple DF, Jr., Hillegass EA, Dudley GA (1999) Influence of complete spinal cord injury on skeletal muscle cross-sectional area within the first 6 months of injury. Eur J Appl Physiol Occup Physiol 80:373-378.

Caudle KL, Brown EH, Shum-Siu A, Burke DA, Magnuson TS, Voor MJ, Magnuson DS (2011) Hindlimb immobilization in a wheelchair alters functional recovery following contusive spinal cord injury in the adult rat. Neurorehabil Neural Repair 25:729-739.

Cazalets JR, Borde M, Clarac F (1995) Localization and organization of the central pattern generator for hindlimb locomotion in newborn rat. $\mathrm{J}$ Neurosci 15:4943-4951. 
Cha J, Heng C, Reinkensmeyer DJ, Roy RR, Edgerton VR, De Leon RD (2007) Locomotor ability in spinal rats is dependent on the amount of activity imposed on the hindlimbs during treadmill training. J Neurotrauma 24:1000-1012.

Cheng H, Almstrom S, Gimenez-Llort L, Chang R, Ove Ogren S, Hoffer B, Olson $L$ (1997) Gait analysis of adult paraplegic rats after spinal cord repair. Exp Neurol 148:544-557.

Cormery B, Beaumont E, Csukly K, Gardiner P (2005) Hindlimb unweighting for 2 weeks alters physiological properties of rat hindlimb motoneurones. $J$ Physiol 568:841-850.

Cote MP, Gossard JP (2004) Step training-dependent plasticity in spinal cutaneous pathways. J Neurosci 24:11317-11327.

Coupaud S, Gollee H, Hunt KJ, Fraser MH, Allan DB, McLean AN (2008) Armcranking exercise assisted by Functional Electrical Stimulation in $\mathrm{C} 6$ tetraplegia: a pilot study. Technol Health Care 16:415-427.

Courtine G, Song B, Roy RR, Zhong H, Herrmann JE, Ao Y, Qi J, Edgerton VR, Sofroniew MV (2008) Recovery of supraspinal control of stepping via indirect propriospinal relay connections after spinal cord injury. Nat Med 14:69-74.

Courtine G, Gerasimenko Y, van den Brand R, Yew A, Musienko P, Zhong H, Song B, Ao Y, Ichiyama RM, Lavrov I, Roy RR, Sofroniew MV, Edgerton VR (2009) Transformation of nonfunctional spinal circuits into functional states after the loss of brain input. Nat Neurosci 12:1333-1342.

Cowley KC, Schmidt BJ (1997) Regional distribution of the locomotor patterngenerating network in the neonatal rat spinal cord. J Neurophysiol 77:247259.

Crown ED, Ferguson AR, Joynes RL, Grau JW (2002) Instrumental learning within the spinal cord. II. Evidence for central mediation. Physiol Behav 77:259-267. 
Dallmeijer AJ, Ottjes L, de Waardt E, van der Woude LH (2004) A physiological comparison of synchronous and asynchronous hand cycling. Int J Sports Med 25:622-626.

Davies AL, Hayes KC, Shi R (2006) Recombinant human TNFalpha induces concentration-dependent and reversible alterations in the electrophysiological properties of axons in mammalian spinal cord. $\mathrm{J}$ Neurotrauma 23:1261-1273.

de Leon RD, Acosta CN (2006) Effect of robotic-assisted treadmill training and chronic quipazine treatment on hindlimb stepping in spinally transected rats. J Neurotrauma 23:1147-1163.

de Leon RD, See PA, Chow CH (2011) Differential effects of low versus high amounts of weight supported treadmill training in spinally transected rats. J Neurotrauma 28:1021-1033.

de Leon RD, Hodgson JA, Roy RR, Edgerton VR (1998) Full Weight-Bearing Hindlimb Standing Following Stand Training in the Adult Spinal Cat. J Neurophysiol 80:83-91.

de Leon RD, Hodgson JA, Roy RR, Edgerton VR (1998b) Locomotor Capacity Attributable to Step Training Versus Spontaneous Recovery After Spinalization in Adult Cats. J Neurophysiol 79:1329-1340.

de Leon RD, Hodgson JA, Roy RR, Edgerton VR (1999a) Retention of Hindlimb Stepping Ability in Adult Spinal Cats After the Cessation of Step Training. J Neurophysiol 81:85-94.

de Leon RD, Tamaki H, Hodgson JA, Roy RR, Edgerton VR (1999b) Hindlimb Locomotor and Postural Training Modulates Glycinergic Inhibition in the Spinal Cord of the Adult Spinal Cat. J Neurophysiol 82:359-369.

Deschenes MR, Giles JA, McCoy RW, Volek JS, Gomez AL, Kraemer WJ (2002) Neural factors account for strength decrements observed after short-term muscle unloading. Am J Physiol Regul Integr Comp Physiol 282:R578583. 
Dietz V, Wirz M, Curt A, Colombo G (1998) Locomotor pattern in paraplegic patients: training effects and recovery of spinal cord function. Spinal Cord 36:380-390.

Ditunno JF, Little JW, Tessler A, Burns AS (2004) Spinal shock revisited: a fourphase model. Spinal Cord 42:383-395.

Dobkin B, Barbeau H, Deforge D, Ditunno J, Elashoff R, Apple D, Basso M, Behrman A, Harkema S, Saulino M, Scott M (2007) The evolution of walking-related outcomes over the first 12 weeks of rehabilitation for incomplete traumatic spinal cord injury: the multicenter randomized Spinal Cord Injury Locomotor Trial. Neurorehabil Neural Repair 21:25-35.

Dobkin B, Apple D, Barbeau H, Basso M, Behrman A, Deforge D, Ditunno J, Dudley G, Elashoff R, Fugate L, Harkema S, Saulino M, Scott M (2006) Weight-supported treadmill vs over-ground training for walking after acute incomplete SCI. Neurology 66:484-493.

Dobkin $\mathrm{BH}$ (2007) Confounders in rehabilitation trials of task-oriented training: lessons from the designs of the EXCITE and SCILT multicenter trials. Neurorehabil Neural Repair 21:3-13.

Dobkin BH, Duncan PW (2012) Should body weight-supported treadmill training and robotic-assistive steppers for locomotor training trot back to the starting gate? Neurorehabil Neural Repair 26:308-317.

Dobkin BH, Harkema S, Requejo P, Edgerton VR (1995) Modulation of locomotor-like EMG activity in subjects with complete and incomplete spinal cord injury. J Neurol Rehabil 9:183-190.

Dolbow DR, Gorgey AS, Daniels JA, Adler RA, Moore JR, Gater DR, Jr. (2011) The effects of spinal cord injury and exercise on bone mass: a literature review. NeuroRehabilitation 29:261-269.

Doyle CA, Hunt SP (1999) Substance P receptor (neurokinin-1)-expressing neurons in lamina $I$ of the spinal cord encode for the intensity of noxious stimulation: a c-Fos study in rat. Neuroscience 89:17-28.

Edgerton VR, Roy RR (1994) Neuromuscular adaptation to actual and simulated weightlessness. Adv Space Biol Med 4:33-67. 
Edgerton VR, Roy RR, Allen DL, Monti RJ (2002) Adaptations in skeletal muscle disuse or decreased-use atrophy. Am J Phys Med Rehabil 81:S127-147.

Edgerton VR, Tillakaratne NJ, Bigbee AJ, de Leon RD, Roy RR (2004) Plasticity of the spinal neural circuitry after injury. Annu Rev Neurosci 27:145-167.

Edgerton VR, Roy RR, Hodgson JA, Prober RJ, de Guzman CP, de Leon R (1991) A physiological basis for the development of rehabilitative strategies for spinally injured patients. J Am Paraplegia Soc 14:150-157.

Edgerton VR, Roy RR, Hodgson JA, Prober RJ, de Guzman CP, de Leon R (1992) Potential of adult mammalian lumbosacral spinal cord to execute and acquire improved locomotion in the absence of supraspinal input. $J$ Neurotrauma 9 Suppl 1:S119-128.

Edgerton VR, de Leon RD, Tillakaratne N, Recktenwald MR, Hodgson JA, Roy RR (1997) Use-dependent plasticity in spinal stepping and standing. Adv Neurol 72:233-247.

Eidelberg E, Walden JG, Nguyen LH (1981) Locomotor control in macaque monkeys. Brain 104:647-663.

Engel P, Hildebrandt G (1974) Wheelchair design--technological and physiological aspects. Proc R Soc Med 67:409-413.

Engesser-Cesar C, Anderson AJ, Basso DM, Edgerton VR, Cotman CW (2005) Voluntary wheel running improves recovery from a moderate spinal cord injury. J Neurotrauma 22:157-171.

Erschbamer MK, Pham TM, Zwart MC, Baumans V, Olson L (2006) Neither environmental enrichment nor voluntary wheel running enhances recovery from incomplete spinal cord injury in rats. Exp Neurol 201:154-164.

Fehlings MG, Tator CH, Linden RD (1989) The relationships among the severity of spinal cord injury, motor and somatosensory evoked potentials and spinal cord blood flow. Electroencephalogr Clin Neurophysiol 74:241-259.

Ferguson AR, Crown ED, Grau JW (2006) Nociceptive plasticity inhibits adaptive learning in the spinal cord. Neuroscience 141:421-431. 
Ferris DP, Aagaard P, Simonsen EB, Farley CT, Dyhre-Poulsen P (2001) Soleus $\mathrm{H}$-reflex gain in humans walking and running under simulated reduced gravity. J Physiol 530:167-180.

Fischer FR, Peduzzi JD (2007) Functional recovery in rats with chronic spinal cord injuries after exposure to an enriched environment. J Spinal Cord Med 30:147-155.

Flynn JR, Graham BA, Galea MP, Callister RJ (2011) The role of propriospinal interneurons in recovery from spinal cord injury. Neuropharmacology 60:809-822.

Forssberg $H$ (1979) Stumbling corrective reaction: a phase-dependent compensatory reaction during locomotion. J Neurophysiol 42:936-953.

Fouad K, Tetzlaff W (2011) Rehabilitative training and plasticity following spinal cord injury. Exp Neurol.

Fouad K, Metz GAS, Merkler D, Dietz V, Schwab ME (2000) Treadmill training in incomplete spinal cord injured rats. Behavioural Brain Research 115:107113.

Frigon A, Rossignol S (2008) Adaptive changes of the locomotor pattern and cutaneous reflexes during locomotion studied in the same cats before and after spinalization. J Physiol 586:2927-2945.

Gerasimenko Y, Roy RR, Edgerton VR (2008) Epidural stimulation: comparison of the spinal circuits that generate and control locomotion in rats, cats and humans. Exp Neurol 209:417-425.

Gerasimenko YP, Ichiyama RM, Lavrov IA, Courtine G, Cai L, Zhong H, Roy RR, Edgerton VR (2007) Epidural spinal cord stimulation plus quipazine administration enable stepping in complete spinal adult rats. $J$ Neurophysiol 98:2525-2536.

Glaser RM, Sawka MN, Young RE, Suryaprasad AG (1980a) Applied physiology for wheelchair design. J Appl Physiol 48:41-44. 
Glaser RM, Sawka MN, Brune MF, Wilde SW (1980b) Physiological responses to maximal effort wheelchair and arm crank ergometry. J Appl Physiol 48:1060-1064.

Gorska T, Bem T, Majczynski H, Zmyslowski W (1993) Unrestrained walking in cats with partial spinal lesions. Brain Res Bull 32:241-249.

Grau JW, Washburn SN, Hook MA, Ferguson AR, Crown ED, Garcia G, Bolding KA, Miranda RC (2004) Uncontrollable stimulation undermines recovery after spinal cord injury. J Neurotrauma 21:1795-1817.

Griesbach GS, Gomez-Pinilla F, Hovda DA (2004) The upregulation of plasticityrelated proteins following $\mathrm{TBI}$ is disrupted with acute voluntary exercise. Brain Res 1016:154-162.

Grill RJ (2005) User-defined variables that affect outcome in spinal cord contusion/compression models. Exp Neurol 196:1-5.

Grillner S, Zangger P (1979) On the central generation of locomotion in the low spinal cat. Exp Brain Res 34:241-261.

Gruner JA (1992) A monitored contusion model of spinal cord injury in the rat. J Neurotrauma 9:123-126; discussion 126-128.

Guertin PA, Ung RV, Rouleau P, Steuer I (2011) Effects on locomotion, muscle, bone, and blood induced by a combination therapy eliciting weight-bearing stepping in nonassisted spinal cord-transected mice. Neurorehabil Neural Repair 25:234-242.

Guissard N, Duchateau J, Hainaut K (2001) Mechanisms of decreased motoneurone excitation during passive muscle stretching. Exp Brain Res 137:163-169.

Hadi B, Zhang YP, Burke DA, Shields CB, Magnuson DS (2000) Lasting paraplegia caused by loss of lumbar spinal cord interneurons in rats: no direct correlation with motor neuron loss. J Neurosurg 93:266-275.

Hamers FP, Lankhorst AJ, van Laar TJ, Veldhuis WB, Gispen WH (2001) Automated quantitative gait analysis during overground locomotion in the 
rat: its application to spinal cord contusion and transection injuries. $J$ Neurotrauma 18:187-201.

Harkema S, Behrman A, Barbeau H (2012a) Evidence-based therapy for recovery of function after spinal cord injury. Handb Clin Neurol 109:259274.

Harkema SJ (2001) Neural plasticity after human spinal cord injury: application of locomotor training to the rehabilitation of walking. Neuroscientist 7:455468.

Harkema SJ (2008) Plasticity of interneuronal networks of the functionally isolated human spinal cord. Brain Res Rev 57:255-264.

Harkema SJ, Schmidt-Read M, Lorenz DJ, Edgerton VR, Behrman AL (2011) Balance and ambulation improvements in individuals with chronic incomplete spinal cord injury using locomotor training-based rehabilitation. Arch Phys Med Rehabil 93:1508-1517.

Harkema SJ, Hurley SL, Patel UK, Requejo PS, Dobkin BH, Edgerton VR (1997) Human Lumbosacral Spinal Cord Interprets Loading During Stepping. J Neurophysiol 77:797-811.

Harkema SJ, Hillyer J, Schmidt-Read M, Ardolino E, Sisto SA, Behrman AL (2012b) Locomotor training: as a treatment of spinal cord injury and in the progression of neurologic rehabilitation. Arch Phys Med Rehabil 93:15881597.

Harvey L (2008) Management of People With Spinal Cord Injuries: A Guide for Physiotherapists. London, England. Elsevier.

Harvey L, Herbert R, Crosbie J (2002) Does stretching induce lasting increases in joint ROM? A systematic review. Physiother Res Int 7:1-13.

Harvey LA, Herbert RD (2002) Muscle stretching for treatment and prevention of contracture in people with spinal cord injury. Spinal Cord 40:1-9.

Harvey LA, McQuade L, Hawthorne S, Byak A (2003a) Quantifying the magnitude of torque physiotherapists apply when stretching the hamstring 
muscles of people with spinal cord injury. Arch Phys Med Rehabil 84:1072-1075.

Harvey LA, Lin CW, Glinsky JV, De Wolf A (2008) The effectiveness of physical interventions for people with spinal cord injuries: a systematic review. Spinal Cord.

Harvey LA, Lin CW, Glinsky JV, De Wolf A (2009) The effectiveness of physical interventions for people with spinal cord injuries: a systematic review. Spinal Cord 47:184-195.

Harvey LA, Glinsky JA, Katalinic OM, Ben M (2011) Contracture management for people with spinal cord injuries. NeuroRehabilitation 28:17-20.

Harvey LA, Batty J, Crosbie J, Poulter S, Herbert RD (2000) A randomized trial assessing the effects of 4 weeks of daily stretching on ankle mobility in patients with spinal cord injuries. Arch Phys Med Rehabil 81:1340-1347.

Harvey LA, Byak AJ, Ostrovskaya M, Glinsky J, Katte L, Herbert RD (2003b) Randomised trial of the effects of four weeks of daily stretch on extensibility of hamstring muscles in people with spinal cord injuries. Aust J Physiother 49:176-181.

Hayes KC, Davies AL, Ashki N, Kramer JK, Close TE (2007) Re: Ditunno JF, Little JW, Tessler A, Burns AS. Spinal shock revisited: a four-phase model. Spinal Cord 2004; 42: 383-395. Spinal Cord 45:395-396.

Heng C, de Leon RD (2008) Treadmill training enhances the recovery of normal stepping patterns in spinal cord contused rats. Exp Neurol.

Hiebert GW, Whelan PJ, Prochazka A, Pearson KG (1996) Contribution of hind limb flexor muscle afferents to the timing of phase transitions in the cat step cycle. J Neurophysiol 75:1126-1137.

Hill RL, Zhang YP, Burke DA, Devries WH, Zhang Y, Magnuson DS, Whittemore SR, Shields CB (2009) Anatomical and functional outcomes following a precise, graded, dorsal laceration spinal cord injury in C57BL/6 mice. J Neurotrauma 26:1-15. 
Hodgson JA, Roy RR, de Leon R, Dobkin B, Edgerton VR (1994) Can the mammalian lumbar spinal cord learn a motor task? Med Sci Sports Exerc 26:1491-1497.

Hodgson JA, Roy RR, Higuchi N, Monti RJ, Zhong H, Grossman E, Edgerton VR (2005) Does daily activity level determine muscle phenotype? J Exp Biol 208:3761-3770.

Hooper SL (2000) Central pattern generators. Curr Biol 10:R176.

Hornby TG, Zemon DH, Campbell D (2005) Robotic-assisted, body-weightsupported treadmill training in individuals following motor incomplete spinal cord injury. Phys Ther 85:52-66.

Hostettler S, Leuthold L, Brechbuhl J, Mueller G, Illi SK, Spengler CM (2012) Maximal cardiac output during arm exercise in the sitting position after cervical spinal cord injury. J Rehabil Med 44:131-136.

Huie JR, Baumbauer KM, Lee KH, Bresnahan JC, Beattie MS, Ferguson AR, Grau JW (2012) Glial tumor necrosis factor alpha (TNFalpha) generates metaplastic inhibition of spinal learning. PLoS One 7:e39751.

Ichiyama RM, Courtine G, Gerasimenko YP, Yang GJ, van den Brand R, Lavrov IA, Zhong H, Roy RR, Edgerton VR (2008) Step training reinforces specific spinal locomotor circuitry in adult spinal rats. J Neurosci 28:73707375 .

Jack LP, Purcell M, Allan DB, Hunt KJ (2010) Comparison of peak cardiopulmonary performance parameters during robotics-assisted treadmill exercise and arm crank ergometry in incomplete spinal cord injury. Technol Health Care 18:285-296.

James ND, Bartus K, Grist J, Bennett DL, McMahon SB, Bradbury EJ (2011) Conduction failure following spinal cord injury: functional and anatomical changes from acute to chronic stages. J Neurosci 31:18543-18555.

Jayaraman A, Shah P, Gregory C, Bowden M, Stevens J, Bishop M, Walter G, Behrman A, Vandenborne K (2008) Locomotor training and muscle function after incomplete spinal cord injury: case series. J Spinal Cord Med 31:185-193. 
Jiang SD, Dai LY, Jiang LS (2006) Osteoporosis after spinal cord injury. Osteoporos Int 17:180-192.

Jiang SD, Jiang LS, Dai LY (2007) Changes in bone mass, bone structure, bone biomechanical properties, and bone metabolism after spinal cord injury: a 6-month longitudinal study in growing rats. Calcif Tissue Int 80:167-175.

Jordan LM, Schmidt BJ (2002) Propriospinal neurons involved in the control of locomotion: potential targets for repair strategies? Prog Brain Res 137:125-139.

Juvin L, Simmers J, Morin D (2005) Propriospinal circuitry underlying interlimb coordination in mammalian quadrupedal locomotion. J Neurosci 25:60256035.

Kakulas BA (1999) A review of the neuropathology of human spinal cord injury with emphasis on special features. J Spinal Cord Med 22:119-124.

Katalinic OM, Harvey LA, Herbert RD (2011) Effectiveness of stretch for the treatment and prevention of contractures in people with neurological conditions: a systematic review. Phys Ther 91:11-24.

Katalinic OM, Harvey LA, Herbert RD, Moseley AM, Lannin NA, Schurr K (2010) Stretch for the treatment and prevention of contractures. Cochrane Database Syst Rev:CD007455.

Kelley G (1996) Mechanical overload and skeletal muscle fiber hyperplasia: a meta-analysis. J Appl Physiol 81:1584-1588.

Khan MA (1986) An ultrastructural study of the stretch-induced hypertrophy of skeletal muscle. Cell Biol Int Rep 10:955-962.

Klimstra MD, Thomas E, Stoloff RH, Ferris DP, Zehr EP (2009) Neuromechanical considerations for incorporating rhythmic arm movement in the rehabilitation of walking. Chaos 19:026102.

Kojima N, Nakazawa K, Yano H (1999) Effects of limb loading on the lower-limb electromyographic activity during orthotic locomotion in a paraplegic patient. Neurosci Lett 274:211-213. 
Konkle AT, Kentner AC, Baker SL, Stewart A, Bielajew C (2010) Environmentalenrichment-related variations in behavioral, biochemical, and physiologic responses of Sprague-Dawley and Long Evans rats. J Am Assoc Lab Anim Sci 49:427-436.

Koopmans GC, Deumens R, Honig WM, Hamers FP, Steinbusch HW, Joosten EA (2005) The assessment of locomotor function in spinal cord injured rats: the importance of objective analysis of coordination. J Neurotrauma 22:214-225.

Kuerzi J, Brown EH, Shum-Siu A, Siu A, Burke D, Morehouse J, Smith RR, Magnuson DS (2010) Task-specificity vs. ceiling effect: step-training in shallow water after spinal cord injury. Exp Neurol 224:178-187.

Kunkel-Bagden E, Dai HN, Bregman BS (1993) Methods to assess the development and recovery of locomotor function after spinal cord injury in rats. Exp Neurol 119:153-164.

Langlet C, Leblond H, Rossignol S (2005) Mid-lumbar segments are needed for the expression of locomotion in chronic spinal cats. J Neurophysiol 93:2474-2488.

Lankhorst AJ, ter Laak MP, van Laar TJ, van Meeteren NL, de Groot JC, Schrama LH, Hamers FP, Gispen WH (2001) Effects of enriched housing on functional recovery after spinal cord contusive injury in the adult rat. $\mathrm{J}$ Neurotrauma 18:203-215.

Leblond H, L'Esperance M, Orsal D, Rossignol S (2003) Treadmill locomotion in the intact and spinal mouse. J Neurosci 23:11411-11419.

Liu D, Zhao CQ, Li H, Jiang SD, Jiang LS, Dai LY (2008a) Effects of spinal cord injury and hindlimb immobilization on sublesional and supralesional bones in young growing rats. Bone 43:119-125.

Liu M, Bose P, Walter GA, Thompson FJ, Vandenborne K (2008b) A longitudinal study of skeletal muscle following spinal cord injury and locomotor training. Spinal Cord 46:488-493. 
Lovely RG, Gregor RJ, Roy RR, Edgerton VR (1986) Effects of training on the recovery of full-weight-bearing stepping in the adult spinal cat. Exp Neurol 92:421-435.

Loy DN, Magnuson DS, Zhang YP, Onifer SM, Mills MD, Cao QL, Darnall JB, Fajardo LC, Burke DA, Whittemore SR (2002a) Functional redundancy of ventral spinal locomotor pathways. J Neurosci 22:315-323.

Magnuson DS, Trinder TC (1997) Locomotor rhythm evoked by ventrolateral funiculus stimulation in the neonatal rat spinal cord in vitro. J Neurophysiol 77:200-206.

Magnuson DS, Lovett R, Coffee C, Gray R, Han Y, Zhang YP, Burke DA (2005a) Functional consequences of lumbar spinal cord contusion injuries in the adult rat. J Neurotrauma 22:529-543.

Magnuson DS, Smith RR, Brown EH, Enzmann G, Angeli C, Quesada PM, Burke D (2009) Swimming as a Model of Task-Specific Locomotor Retraining After Spinal Cord Injury in the Rat. Neurorehabil Neural Repair.

Magnuson DSK, Trinder TC, Zhang YP, Burke D, Morassutti DJ, Shields CB (1999) Comparing Deficits Following Excitotoxic and Contusion Injuries in the Thoracic and Lumbar Spinal Cord of the Adult Rat. Experimental Neurology 156:191-204.

Magnuson DSK, Lovett R, Coffee C, Gray R, Han Y, Zhang YP, Burke DA (2005b) Functional Consequences of Lumbar Spinal Cord Contusion Injuries in the Adult Rat. Journal of Neurotrauma 22:529-543.

Maier IC, Baumann K, Thallmair M, Weinmann O, Scholl J, Schwab ME (2008) Constraint-induced movement therapy in the adult rat after unilateral corticospinal tract injury. J Neurosci 28:9386-9403.

Maier IC, Ichiyama RM, Courtine G, Schnell L, Lavrov I, Edgerton VR, Schwab ME (2009) Differential effects of anti-Nogo-A antibody treatment and treadmill training in rats with incomplete spinal cord injury. Brain 132:14261440.

Majczynski H, Slawinska U (2007) Locomotor recovery after thoracic spinal cord lesions in cats, rats and humans. Acta Neurobiol Exp (Wars) 67:235-257. 
Marsh BC, Astill SL, Utley A, Ichiyama RM (2011) Movement rehabilitation after spinal cord injuries: emerging concepts and future directions. Brain Res Bull 84:327-336.

Martinez M, Rossignol S (2011) Changes in CNS structures after spinal cord lesions implications for BMI. Prog Brain Res 194:191-202.

Masi L (2012) Crosstalk between the brain and bone. Clin Cases Miner Bone Metab 9:13-16.

McKinley W, Santos K, Meade M, Brooke K (2007) Incidence and outcomes of spinal cord injury clinical syndromes. J Spinal Cord Med 30:215-224.

Morey-Holton ER, Globus RK (2002) Hindlimb unloading rodent model: technical aspects. J Appl Physiol 92:1367-1377.

Morita H, Crone C, Christenhuis D, Petersen NT, Nielsen JB (2001) Modulation of presynaptic inhibition and disynaptic reciprocal la inhibition during voluntary movement in spasticity. Brain 124:826-837.

Moriyama H, Yoshimura O, Sunahori H, Tobimatsu Y (2006) Comparison of muscular and articular factors in the progression of contractures after spinal cord injury in rats. Spinal Cord 44:174-181.

Moriyama H, Yoshimura O, Kawamata S, Takemoto H, Saka Y, Tobimatsu Y (2007) Alteration of Knee Joint Connective Tissues during Contracture Formation in Spastic Rats after an Experimentally Induced Spinal Cord Injury. Connective Tissue Research 48:180 - 187.

Morse LR, Xu Y, Solomon B, Boyle L, Yoganathan S, Stashenko P, Battaglino RA (2011) Severe Spinal Cord Injury Causes Immediate Multi-cellular Dysfunction at the Chondro-Osseous Junction. TransI Stroke Res 2:643650.

Mossberg K, Willman C, Topor MA, Crook H, Patak S (1999) Comparison of asynchronous versus synchronous arm crank ergometry. Spinal Cord $37: 569-574$. 
Muir GD, Steeves JD (1995) Phasic cutaneous input facilitates locomotor recovery after incomplete spinal injury in the chick. J Neurophysiol 74:358368.

Mulloney B, Smarandache C (2010) Fifty Years of CPGs: Two Neuroethological Papers that Shaped the Course of Neuroscience. Front Behav Neurosci 4.

Multon S, Franzen R, Poirrier AL, Scholtes F, Schoenen J (2003) The effect of treadmill training on motor recovery after a partial spinal cord compression-injury in the adult rat. J Neurotrauma 20:699-706.

Nadeau S, Jacquemin G, Fournier C, Lamarre Y, Rossignol S (2010) Spontaneous motor rhythms of the back and legs in a patient with a complete spinal cord transection. Neurorehabil Neural Repair 24:377-383.

Nichols TR, Cope TC, Abelew TA (1999) Rapid spinal mechanisms of motor coordination. Exerc Sport Sci Rev 27:255-284.

Norenberg MD, Smith J, Marcillo A (2004) The pathology of human spinal cord injury: defining the problems. J Neurotrauma 21:429-440.

O'Sullivan, Schmitz (2001) Guide to Physical Therapist Practice. Second Edition. American Physical Therapy Association. Phys Ther 81:9-746.

Oyinbo CA (2011) Secondary injury mechanisms in traumatic spinal cord injury: a nugget of this multiply cascade. Acta Neurobiol Exp (Wars) 71:281-299.

Patton BC, Hook MA, Ferguson AR, Crown ED, Grau JW (2004) The behavioral deficit observed following noncontingent shock in spinalized rats is prevented by the protein synthesis inhibitor cycloheximide. Behav Neurosci 118:653-658.

Poon PC, Gupta D, Shoichet MS, Tator CH (2007) Clip compression model is useful for thoracic spinal cord injuries: histologic and functional correlates. Spine (Phila Pa 1976) 32:2853-2859.

Quevedo J, Stecina K, Gosgnach S, McCrea DA (2005) Stumbling corrective reaction during fictive locomotion in the cat. J Neurophysiol 94:2045-2052. 
Radford JA, Burns J, Buchbinder R, Landorf KB, Cook C (2006) Does stretching increase ankle dorsiflexion range of motion? A systematic review. $\mathrm{Br} \mathrm{J}$ Sports Med 40:870-875; discussion 875.

Reed WR, Shum-Siu A, Magnuson DSK (2008) Reticulospinal pathways in the ventrolateral funiculus with terminations in the cervical and lumbar enlargements of the adult rat spinal cord. Neuroscience 151:505-517.

Reed WR, Shum-Siu A, Whelan A, Onifer SM, Magnuson DS (2009) Anterograde labeling of ventrolateral funiculus pathways with spinal enlargement connections in the adult rat spinal cord. Brain Res 1302:7684.

Requejo PS, Lee SE, Mulroy SJ, Haubert LL, Bontrager EL, Gronley JK, Perry J (2008) Shoulder muscular demand during lever-activated vs pushrim wheelchair propulsion in persons with spinal cord injury. J Spinal Cord Med 31:568-577.

Robert AA, Al Jadid MS, Bin Afif S, Al Sowyed AA, Al-Mubarak S (2010) The effects of different rehabilitation strategies on the functional recovery of spinal cord injured rats: an experimental study. Spine (Phila Pa 1976) 35:E1273-1277.

Rosenzweig ES, Courtine G, Jindrich DL, Brock JH, Ferguson AR, Strand SC, Nout YS, Roy RR, Miller DM, Beattie MS, Havton LA, Bresnahan JC, Edgerton VR, Tuszynski MH (2010) Extensive spontaneous plasticity of corticospinal projections after primate spinal cord injury. Nat Neurosci 13:1505-1510.

Rossignol S (1996) Neural control of stereotypic limb movements. Handbook of Physiology, Section 12 Exercise: Regulation and Integration of Multiple Systems, edited by LB Rowell and JT Shepherd:173-216.

Rossignol S, Drew T, Brustein E, Jiang W (1999) Locomotor performance and adaptation after partial or complete spinal cord lesions in the cat. Prog Brain Res 123:349-365.

Rossignol S, Bouyer L, BarthÈlemy D, Langlet C, Leblond H (2002) Recovery of locomotion in the cat following spinal cord lesions. Brain Research Reviews 40:257-266. 
Rossignol S, Chau C, Brustein E, Belanger M, Barbeau H, Drew T (1996) Locomotor capacities after complete and partial lesions of the spinal cord. Acta Neurobiol Exp (Wars) 56:449-463.

Rossignol S, Bouyer L, Langlet C, Barthelemy D, Chau C, Giroux N, Brustein E, Marcoux J, Leblond H, Reader TA (2004) Determinants of locomotor recovery after spinal injury in the cat. Prog Brain Res 143:163-172.

Roy RR, Baldwin KM, Edgerton VR (1991) The plasticity of skeletal muscle: effects of neuromuscular activity. Exerc Sport Sci Rev 19:269-312.

Roy RR, Hodgson JA, Lauretz SD, Pierotti DJ, Gayek RJ, Edgerton VR (1992) Chronic spinal cord-injured cats: surgical procedures and management. Lab Anim Sci 42:335-343.

Roy RR, Pierotti DJ, Baldwin KM, Zhong H, Hodgson JA, Edgerton VR (1998) Cyclical passive stretch influences the mechanical properties of the inactive cat soleus. Exp Physiol 83:377-385.

Roy RR, Zhong H, Khalili N, Kim SJ, Higuchi N, Monti RJ, Grossman E, Hodgson JA, Edgerton VR (2007) Is spinal cord isolation a good model of muscle disuse? Muscle Nerve 35:312-321.

Rubin C, Turner AS, Bain S, Mallinckrodt C, McLeod K (2001) Anabolism. Low mechanical signals strengthen long bones. Nature 412:603-604.

Scheff SW, Rabchevsky AG, Fugaccia I, Main JA, Lumpp JE, Jr. (2003) Experimental modeling of spinal cord injury: characterization of a forcedefined injury device. J Neurotrauma 20:179-193.

Schucht P, Raineteau O, Schwab ME, Fouad K (2002) Anatomical correlates of locomotor recovery following dorsal and ventral lesions of the rat spinal cord. Exp Neurol 176:143-153.

Segal RL, Lewek MD, McCulloch K, Mercer VS (2011) The necessity for effective interaction between basic scientists and rehabilitation clinicians. Cells Tissues Organs 193:290-297. 
Shah PK, Stevens JE, Gregory CM, Pathare NC, Jayaraman A, Bickel SC, Bowden M, Behrman AL, Walter GA, Dudley GA, Vandenborne K (2006) Lower-extremity muscle cross-sectional area after incomplete spinal cord injury. Arch Phys Med Rehabil 87:772-778.

Shoji H, Mizoguchi K (2010) Acute and repeated stress differentially regulates behavioral, endocrine, neural parameters relevant to emotional and stress response in young and aged rats. Behav Brain Res 211:169-177.

Siegel, Castellan (1988) Nonparametric Statistics. 2nd ED. Boston: McGraw Hill.

Siegenthaler MM, Berchtold NC, Cotman CW, Keirstead HS (2008) Voluntary running attenuates age-related deficits following SCI. Exp Neurol 210:207216.

Singh A, Murray M, Houle JD (2011) A training paradigm to enhance motor recovery in contused rats: effects of staircase training. Neurorehabil Neural Repair 25:24-34.

Slawinska U, Rossignol S, Bennett DJ, Schmidt BJ, Frigon A, Fouad K, Jordan LM (2012) Comment on "Restoring voluntary control of locomotion after paralyzing spinal cord injury". Science 338:328; author reply 328 .

Smarick SD, Rylander H, Burkitt JM, Scott NE, Woelz JS, Jandrey KE, Aldrich J, Sturges BK (2007) Treatment of traumatic cervical myelopathy with surgery, prolonged positive-pressure ventilation, and physical therapy in a dog. J Am Vet Med Assoc 230:370-374.

Smith KJ, Blakemore WF, McDonald WI (1979) Central remyelination restores secure conduction. Nature 280:395-396.

Smith R, Brown EH, Shum-Siu A, Whelan A, Burke D, Benton RL, Magnuson DS (2009) Swim training initiated acutely after spinal cord injury is ineffective and induces extravasation in and around the epicenter. J Neurotrauma.

Smith RR, Shum-Siu A, Baltzley R, Bunger M, Baldini A, Burke DA, Magnuson DSK (2006a) Effects of Swimming on Functional Recovery after Incomplete Spinal Cord Injury in Rats. Journal of Neurotrauma 23:908919. 
Smith RR, Burke DA, Baldini AD, Shum-Siu A, Baltzley R, Bunger M, Magnuson DSK (2006b) The Louisville Swim Scale: A Novel Assessment of Hindlimb Function following Spinal Cord Injury in Adult Rats. Journal of Neurotrauma 23:1654-1670.

Speciale J, Fingeroth JM (2000) Use of physiatry as the sole treatment for three paretic or paralyzed dogs with chronic compressive conditions of the caudal portion of the cervical spinal cord. J Am Vet Med Assoc 217:43-47, 29.

Stevens JE, Liu M, Bose P, O'Steen WA, Thompson FJ, Anderson DK, Vandenborne $\mathrm{K}$ (2006) Changes in soleus muscle function and fiber morphology with one week of locomotor training in spinal cord contusion injured rats. J Neurotrauma 23:1671-1681.

Stokes BT (1992) Experimental spinal cord injury: a dynamic and verifiable injury device. J Neurotrauma 9:129-131; discussion 131-124.

Stokes BT, Noyes DH, Behrmann DL (1992) An electromechanical spinal injury technique with dynamic sensitivity. J Neurotrauma 9:187-195.

Tator $\mathrm{CH}$ (2006) Review of treatment trials in human spinal cord injury: issues, difficulties, and recommendations. Neurosurgery 59:957-982; discussion 982-957.

Tetzlaff W, Fouad K, Kwon B (2009) Be careful what you train for. Nat Neurosci 12:1077-1079.

Thota A, Carlson S, Jung R (2001) Recovery of locomotor function after treadmill training of incomplete spinal cord injured rats. Biomed Sci Instrum 37:6367.

Tillakaratne NJ, de Leon RD, Hoang TX, Roy RR, Edgerton VR, Tobin AJ (2002) Use-dependent modulation of inhibitory capacity in the feline lumbar spinal cord. J Neurosci 22:3130-3143.

Timoszyk WK, Nessler JA, Acosta C, Roy RR, Edgerton VR, Reinkensmeyer DJ, de Leon R (2005) Hindlimb loading determines stepping quantity and quality following spinal cord transection. Brain Res 1050:180-189. 
Trudel G, Zhou J, Uhthoff HK, Laneuville O (2008) Four weeks of mobility after 8 weeks of immobility fails to restore normal motion: a preliminary study. Clin Orthop Relat Res 466:1239-1244.

van den Brand R, Heutschi J, Barraud Q, DiGiovanna J, Bartholdi K, Huerlimann M, Friedli L, Vollenweider I, Moraud EM, Duis S, Dominici N, Micera S, Musienko P, Courtine G (2012) Restoring voluntary control of locomotion after paralyzing spinal cord injury. Science 336:1182-1185.

van der Woude LH, Veeger HE, de Boer Y, Rozendal RH (1993) Physiological evaluation of a newly designed lever mechanism for wheelchairs. J Med Eng Technol 17:232-240.

van der Woude LH, Veeger HE, Dallmeijer AJ, Janssen TW, Rozendaal LA (2001) Biomechanics and physiology in active manual wheelchair propulsion. Med Eng Phys 23:713-733.

van der Woude LH, Horstman A, Faas P, Mechielsen S, Bafghi HA, de Koning JJ (2008) Power output and metabolic cost of synchronous and asynchronous submaximal and peak level hand cycling on a motor driven treadmill in able-bodied male subjects. Med Eng Phys 30:574-580.

Van Meeteren NL, Eggers R, Lankhorst AJ, Gispen WH, Hamers FP (2003) Locomotor recovery after spinal cord contusion injury in rats is improved by spontaneous exercise. J Neurotrauma 20:1029-1037.

Vilensky JA, Moore AM, Eidelberg E, Walden JG (1992) Recovery of Locomotion in Monkeys With Spinal Cord Lesions. J Mot Behav 24:288-296.

Voor MJ, Yang S, Burden RL, Waddell SW (2008) In vivo micro-CT scanning of a rabbit distal femur: repeatability and reproducibility. J Biomech 41:186193.

Voor MJ, Brown EH, Xu Q, Waddell SW, Burden RL, Jr., Burke DA, Magnuson DS (2012) Bone loss following spinal cord injury in a rat model. $J$ Neurotrauma 29:1676-1682.

Warraich Z, Kleim JA (2010) Neural plasticity: the biological substrate for neurorehabilitation. PM R 2:S208-219. 
Wernig A, Muller S (1992) Laufband locomotion with body weight support improved walking in persons with severe spinal cord injuries. Paraplegia 30:229-238.

Wernig A, Nanassy A, Muller S (1999) Laufband (treadmill) therapy in incomplete paraplegia and tetraplegia. J Neurotrauma 16:719-726.

Wernig A, Muller S, Nanassy A, Cagol E (1995) Laufband therapy based on 'rules of spinal locomotion' is effective in spinal cord injured persons. Eur $\mathrm{J}$ Neurosci 7:823-829.

Wessels M, Lucas C, Eriks I, de Groot S (2010) Body weight-supported gait training for restoration of walking in people with an incomplete spinal cord injury: a systematic review. J Rehabil Med 42:513-519.

Windhorst U (2007) Muscle proprioceptive feedback and spinal networks. Brain Res Bull 73:155-202.

Wirz M, Zemon DH, Rupp R, Scheel A, Colombo G, Dietz V, Hornby TG (2005) Effectiveness of automated locomotor training in patients with chronic incomplete spinal cord injury: a multicenter trial. Arch Phys Med Rehabil 86:672-680.

Xu (2008) Evaluation of Bone Quality Using Novel Image Analyses and Mechanical Testing Methods. [PhD Dissertation, University of Louisville, Dept. Mechanical Engineering].

Ye F, Baligand C, Keener JE, Vohra RS, Lim W, Ruhella A, Bose P, Daniels M, Walter GA, Thompson FJP, Vandenborne K (2012) Hindlimb muscle morphology and function in a new atrophy model combining spinal cord injury and cast immobilization. J Neurotrauma.

Zaidi M (2005) Neural surveillance of skeletal homeostasis. Cell Metab 1:219221.

Zehr EP (2005) Neural control of rhythmic human movement: the common core hypothesis. Exerc Sport Sci Rev 33:54-60. 
Zehr EP, Chua R (2000) Modulation of human cutaneous reflexes during rhythmic cyclical arm movement. Exp Brain Res 135:241-250.

Zhang LQ, Chung SG, Bai Z, Xu D, van Rey EM, Rogers MW, Johnson ME, Roth EJ (2002) Intelligent stretching of ankle joints with contracture/spasticity. IEEE Trans Neural Syst Rehabil Eng 10:149-157.

Zhang Y, Ji SR, Wu CY, Fan XH, Zhou HJ, Liu GL (2007) Observation of locomotor functional recovery in adult complete spinal rats with BWSTT using semiquantitative and qualitative methods. Spinal Cord 45:496-501.

Zhang YP, Onifer SM, Burke DA, Shields CB (2001) A topical mixture for preventing, abolishing, and treating autophagia and self-mutilation in laboratory rats. Contemp Top Lab Anim Sci 40:35-36.

Zhang YP, lannotti C, Shields LB, Han Y, Burke DA, Xu XM, Shields CB (2004) Dural closure, cord approximation, and clot removal: enhancement of tissue sparing in a novel laceration spinal cord injury model. J Neurosurg 100:343-352.

Zhang YP, Burke DA, Shields LB, Chekmenev SY, Dincman T, Zhang Y, Zheng $Y$, Smith RR, Benton RL, DeVries WH, Hu X, Magnuson DS, Whittemore SR, Shields CB (2008) Spinal cord contusion based on precise vertebral stabilization and tissue displacement measured by combined assessment to discriminate small functional differences. J Neurotrauma 25:1227-1240. 


\section{CURRICULUM VITAE}

Krista L. Caudle

1054 Garvin PI. \#3, Louisville, KY 40203

krista.caudle@yahoo.com, 502-552-0455

\section{EDUCATION}

University of Louisville, Ph.D. candidacy, defense Fall semester 2012

University of Louisville, M.S. Anatomical Sciences and Neurobiology 2008

University of North Carolina at Charlotte, B.S. Biology 2006

University of North Carolina at Charlotte, B.S. Psych., M. Neuroscience 2004

\section{PUBLICATIONS}

Peer Reviewed

Caudle KL, Brown EH, Shum-Siu A, Burke DA, Magnuson TSG, Voor MJ, and Magnuson DSK

Hindlimb immobilization in a wheelchair alters functional recovery following contusive spinal cord injury in the adult rat. Neurorehabil and Neural Repair. Oct; 25(8):729-39.

Manuscripts in Preparation

Caudle KL, Donaldson KM, Fligor RF, Kumar A, Brown EH, Shum-Siu A, Morehouse JR, Burke DA, Voor MJ and Magnuson DSK.

Functional locomotor recovery is altered in a task-specific manner following contusive $\mathrm{SCl}$ in the adult rat.

Caudle KL, Atkinson D, Brown EH, Chea T, Smith E, Chung K, Cron CC, Shum-Siu A, Burke DA and Magnuson DSK.

Passive-static stretch of hindlimb muscle negatively effects functional recovery following contusive $\mathrm{SCl}$ in the adult rat.

\section{PROFESSIONAL MEMBERSHIPS}

National Neurotrauma Society

2008-present

Society for Neuroscience

2003-present 


\section{FUNDING}

Graduate Student Fellowship

Integrated Program in Biomedical Sciences,

2006-present

Department of Anatomical Sciences and Neurobiology

Funding for my dissertation project provided by David Magnuson, Ph.D. through the Kentucky Spinal Cord and Head Injury Research Trust (KSCHIRT), National Institutes of Health and COBRE.

\section{PRESENTATION HONORS}

Invited Oral Presentations

Kentucky Spinal Cord \& Head Injury Research Trust (KSCHIRT) Symposium 2012 Scholars in Training Session, Embassy Suites Hotel, Lexington, KY

"There is a negative effect of passive hindlimb stretch on locomotor recovery after moderate contusive $\mathrm{SCl}$ in the rat: Could standard of care be maladaptive for locomotion?"

National Neurotrauma Symposium, Abstracts $28^{\text {th }}$ Annual meeting Open Communications: Experimental SCl,

Paris Las Vegas Hotel, Las Vegas, NV

National Neurotrauma Society \$250 Travel Grant Award

"Twenty-four hour wheelchair immobilization after incomplete $\mathrm{SCl}$ in the rat."

Poster Presentation Awards

Excellence in Neuroscience Research, Annual Neuroscience Day

2009

Louisville Chapter of the Society for Neuroscience

First Place Graduate Student Poster, \$500 Travel Grant Award

Research! Louisville, Annual Celebration of Health Related Research

First Place Doctoral Basic-Science Graduate Student Poster,

$\$ 500$ Travel Grant Award

\section{ADDITIONAL ACADEMIC RESEARCH AND TRAINING}

Graduate Research Assistant, Human Locomotion Research Center summer 2007 Frazier Rehab Institute, Louisville, KY

Mentor Susan Harkema, Ph.D., University of Louisville

Project title: Clonus after $\mathrm{SCl}$ in Human 
- Assisted with experiments on an adult using body weight supported treadmill locomotor training

- Analyzed electromyographic data using LabVIEW software

- Processed kinematic locomotor data using Motion Analysis software

Research Assistant, Department of Biology

2003-2006

Mentor David Bashor, Ph.D., University of North Carolina at Charlotte

Caudle KL and Bashor DP 2004. Accommodation and post-inhibitory rebound in Hill-Kernell-MacGregor neuron models. New Orleans, LA: Society for Neuroscience Annual Meeting.

Soc. Neuroscience Abstr. 30:420.9.

- Used neuron simulation models to investigate effects of threshold, time constant, and synapse distribution on motor neuron spiking

- Studied central pattern generator for locomotion using models of multiple neuron populations

- Processed and analyzed data, submitted technical reports, and presented posters

Research Assistant in Neuropsychology, Department of Psychology

Mentor George Demakis, Ph.D., University of North Carolina at Charlotte

- Administered and scored neuropsychological tests for undergraduate volunteers with and without history of mild traumatic brain injury (mTBI)

North Carolina State 4-H Horse Judging Team Member 1995-1998

Mentor Robert Mowrey, Ph.D., North Carolina State University

- Evaluated gait, coordination, physical conformation, and muscle characteristics

- Formulated and delivered persuasive oral presentations to a panel of professionals using specific industry and breed terminology 